\title{
THE SCHRÖDINGER EQUATION WITH A QUASI-PERIODIC POTENTIAL
}

\author{
STEVE SURACE, JR.
}

Abstract. We consider the Schrödinger equation

$$
-\frac{d^{2}}{d x^{2}} \psi+\varepsilon(\cos x+\cos (\alpha x+\vartheta)) \psi=E \psi
$$

where $\varepsilon$ is small and $\alpha$ satisfies the Diophantine inequality

$$
|p+q \alpha| \geq C / q^{2} \text { for } p, q \in \mathbf{Z}, q \neq 0 .
$$

We look for solutions of the form

$$
\psi(x)=e^{i K x} q(x)=e^{i K x} \sum \psi_{m n} e^{i n x} e^{i m(\alpha x+\vartheta)} .
$$

If we try to solve for $\psi=\psi_{m n}$ we are led to the Schrödinger equation on the lattice $\mathbf{Z}^{2}$

$$
H(K) \psi=(\varepsilon \Delta+V(K)) \psi=E \psi
$$

where $\Delta$ is the discrete Laplacian (without diagonal terms) and $V(K)$ is some potential on $\mathbf{Z}^{2}$. We have two main results:

(1) For $\varepsilon$ sufficiently small, $H(K)$ has pure point spectrum for almost every K .

(2) For $\varepsilon$ sufficiently small, the operator

$$
-d^{2} / d x^{2}+\varepsilon(\cos x+\cos (\alpha x+\vartheta))
$$

has no point spectrum.

To prove our results, we must get decay estimates on the Green's function $(E-H)^{-1}$. The decay of the eigenfunction follows from this. In general, we must keep track of small divisors which can make the Green's function large. This is accomplished by a KAM (Kolmogorov, Arnold, Moser) type of multiscale perturbation analysis.

\section{INTRODUCTION}

\section{We consider the Schrödinger equation}

$$
-\frac{d^{2}}{d x^{2}} \psi+\varepsilon(\cos x+\cos (\alpha x+\vartheta)) \psi=E^{*} \psi
$$

where $\varepsilon$ is small and $\alpha$ satisfies the Diophantine inequality

$$
|p+q \alpha| \geq C / q^{2} \text { for } p, q \in \mathbf{Z}, q \neq 0 .
$$

Received by the editors September 30, 1988.

1980 Mathematics Subject Classification (1985 Revision). Primary 34B25; Secondary 34D10. 
The set of irrationals $\alpha$ satisfying the above condition for some constant $C$ has full measure.

If the term $\cos (\alpha x+\vartheta)$ is absent from (0.1), then the potential is periodic and the spectrum is known to be purely absolutely continuous. Moreover, any polynomially bounded solution can be expressed as a linear combination of functions of the form

$$
\psi(x)=e^{i K x} \sum \psi_{n} e^{i n x}
$$

where the coefficients $\psi_{n}$ decay exponentially fast as $n \rightarrow \infty[2,17]$. For a general quasi-periodic potential, Dinaburg and Sinai [4] proved the existence of "Bloch type" eigenfunctions $\psi(x)=e^{i K x} q(x)$ where $q(x)$ is quasi-periodic. By definition, a quasi-periodic function is a function $q(x)$ which can be expressed as

$$
q(x)=F\left(\omega_{1} x, \ldots, \omega_{d} x\right)=F(\omega x)
$$

where $F$ is continuous and periodic in all $d$ variables. To prove our results we usually assume that the frequencies $\omega=\left(\omega_{1}, \ldots, \omega_{d}\right)$ satisfy some Diophantine inequality such as $|\langle j, \omega\rangle|^{-1} \leq C|j|^{s}$ for $j \in \mathbf{Z}^{d}, j \neq 0$.

With any quasi-periodic potential $v(x)$ we can associate a rotation number

$$
\beta\left(E^{*}\right)=\lim _{x \rightarrow \infty} \frac{1}{x} \arg \left(\psi^{\prime}+i \psi\right)
$$

where $\psi$ is a solution of the differential equation $(-\Delta+v) \psi=E^{*} \psi$. In [9] it was shown that $\beta\left(E^{*}\right)=\frac{1}{2}\langle j, \omega\rangle$ for $E^{*}$ in the resolvent set. Dinaburg and Sinai proved their result for a set of rotation numbers "not too close" to $\frac{1}{2}\langle j, \omega\rangle$. Their proof uses KAM (Kolmogorov, Arnold, Moser) type methods. They also established the existence of some absolutely continuous spectrum but did not exclude the presence of point spectrum or singular continuous spectrum.

In our case $(0.1)$ it is natural to try to write

$$
\psi(x)=e^{i K^{*} x} \sum \psi_{m n} e^{i n x} e^{i m(\alpha x+\vartheta)} .
$$

If we assume a solution of this form, we get a recursion formula for the coefficients $\psi_{m n}$

$$
\varepsilon\left[\psi_{m+1, n}+\psi_{m-1, n}+\psi_{m, n+1}+\psi_{m, n-1}\right]+\left(n+m \alpha+K^{*}\right)^{2} \psi_{m n}=E^{*} \psi_{m n} .
$$

Remark. This equation is independent of $\vartheta$.

We now think of $\psi=\psi(m, n)$ as a function on $\mathbf{Z}^{2}$ and write a matrix version of the recursion formula

$$
H \psi \equiv(\varepsilon \Delta+V) \psi=E^{*} \psi
$$

where $\Delta$ is the finite difference Laplacian (without diagonal terms) and $V$ is our lattice potential. The matrix elements are given by

$$
\begin{gathered}
\Delta_{i j}= \begin{cases}1 & \text { if }|i-j|=1, \\
0 & \text { otherwise },\end{cases} \\
V_{i j}=\delta_{i j} v_{j} \text { where } v_{j}=\left(j_{2}+j_{1} \alpha+K^{*}\right)^{2},
\end{gathered}
$$


and $j=\left(j_{1}, j_{2}\right) \in \mathbf{Z}^{2}$. For a more convenient notation we define

$$
[j] \equiv j_{2}+j_{1} \alpha .
$$

With this notation we have

$$
v_{j}=\left([j]+K^{*}\right)^{2}
$$

and by the Diophantine condition on $\alpha$ we have

$$
|[j]| \geq C /|j|^{2} \text { for } j \neq 0 \text {. }
$$

To prove our results we must examine the eigenvalues and eigenfunctions of the lattice operator $H(K)=\varepsilon \Delta+V(K)$. We now list our main results.

Theorem 1. For $\varepsilon$ sufficiently small, $H(K)$ has pure point spectrum for almost every $K$.

Theorem 2. For $\varepsilon$ sufficiently small, the operator

$$
-d^{2} / d x^{2}+\varepsilon(\cos x+\cos (\alpha x+\vartheta))
$$

has no point spectrum.

Remark. For $\varepsilon$ large it has been shown that there is pure point spectrum at low energy for almost every $\vartheta[16]$.

Conjecture. We believe that the spectrum of the operator

$$
-d^{2} / d x^{2}+\varepsilon(\cos x+\cos (\alpha x+\vartheta))
$$

is purely absolutely continuous when $\varepsilon$ is small.

Most of this paper is devoted to the proof of the following lemma, from which the proof of Theorem 1 follows. See [1].

Main Lemma. For $\varepsilon$ sufficiently small and for almost every $K$, every polynomially bounded eigenfunction of the operator $H(K)$ decays exponentially fast.

Other problems of this type on the lattice have recently been studied. In the case where $\left\{v_{j}\right\}$ are independent random variables it was shown that with probability one the spectrum of $H$ is pure point with eigenfunctions which decay exponentially fast $[3,5,6,8,13]$. When $v_{j}(\vartheta)=\cos 2 \pi(\alpha j+\vartheta)$ the same result was shown to be true for almost every $\vartheta[14,16]$. Our methods are closely related to those of [16]. In general, we must deal with the appearance of small divisors in the Green's function $\left(H-E^{*}\right)^{-1}$. To overcome this problem, we will use a multiscale perturbation analysis similar to the one used in the random variable case. Our methods also apply if the potential $v_{j}=([j]+K)^{2}$ is replaced by any other symmetric $C^{2}$ potential with a nondegenerate critical point.

The main idea of the proof of the main lemma is to keep track of the sites in $\mathbf{Z}^{2}$ where $\psi$ may be large. To do this, we will define a sequence of singular sets $S_{n}$ (for $n \geq 0$ ). We list some important properties that $S_{n}$ will have:

(1) $\psi$ decays exponentially fast outside $S_{n}$.

(2) The sites in $S_{n}$ become increasingly sparse as $n$ gets larger. 
Remark. As $n \rightarrow \infty$ we are left with a set $S_{\infty}$ which contains all the sites where $\psi$ may be large; moreover, $\psi$ decays exponentially fast outside $S_{\infty}$.

We now give a brief discussion of $S_{0}$. If we examine the eigenvalue equation $H\left(K^{*}\right) \psi=\left(\varepsilon \Delta+V\left(K^{*}\right)\right) \psi=E^{*} \psi$, we see that $\psi\left(c_{0}^{i}\right)$ may be large at the sites $c_{0}^{i} \in \mathbf{Z}^{2}$ where the potential $v\left(c_{0}^{i}\right)$ is near $E^{*}$. For convenience we define

$$
E_{0}^{i}\left(K^{*}\right)=\left(\left[c_{0}^{i}\right]+K^{*}\right)^{2}=v\left(c_{0}^{i}, K^{*}\right)
$$

to be the value of the potential at $c_{0}^{i}$. Then we define the 0 th order singular set to be

$$
S_{0}\left(K^{*}, E^{*}\right)=\left\{c_{0}^{i} \in \mathbf{Z}^{2}:\left|E_{0}^{i}\left(K^{*}\right)-E^{*}\right| \leq \delta_{0} \sqrt{E^{*}+1}\right\}
$$

where $\delta_{0} \cong \exp \left(-l_{0}^{2 / 3}\right)$ is some small number to be chosen later by making $l_{0}$ as large as we want. The following theorem is the key estimate needed to prove our results. We will state it here for $S_{0}\left(K^{*}, E^{*}\right)$ and later we will prove that it holds true (with the appropriate change of constants) for every singular set $S_{n}\left(K^{*}, E^{*}\right)$. (See $\S 3$ for the general proof.) In $\S 4$ we will show how the Center Theorem is used to prove our main theorems.

Center Theorem. If $c_{0}^{r} \in S_{0}\left(K^{*}, E^{*}\right)$ for $r=i, j$ then

$$
m\left(c_{0}^{i}, c_{0}^{j}\right) \leq 2 \delta_{0}^{1 / 2}
$$

where

$$
m\left(c_{0}^{i}, c_{0}^{j}\right) \equiv \min \left(\left|\left[c_{0}^{i}\right]-\left[c_{0}^{j}\right]\right|,\left|\left[c_{0}^{i}\right]+\left[c_{0}^{j}\right]+2 K^{*}\right|\right) .
$$

We can see the importance of $m\left(c_{0}^{i}, c_{0}^{j}\right)$ if we note that

$$
\begin{aligned}
\left|E_{0}^{i}(K)-E_{0}^{j}(K)\right| & =\left|v\left(c_{0}^{i}, K\right)-v\left(c_{0}^{j}, K\right)\right| \\
& =\left|\left[c_{0}^{i}\right]-\left[c_{0}^{j}\right]\right|\left[c_{0}^{i}\right]+\left[c_{0}^{j}\right]+2 K \mid .
\end{aligned}
$$

The Center Theorem has two important corollaries which will give us information about the structure of $S_{0}\left(K^{*}, E^{*}\right)$.

Corollary 1. Let $s_{0}=\min _{S_{0}}\left|c_{0}^{i}-c_{0}^{j}\right| \equiv\left|c_{0}^{I}-c_{0}^{J}\right|$ and suppose that $s_{0} \leq 6 l_{0}^{2}$. Then every point $c_{0}^{i} \in S_{0}\left(K^{*}, E^{*}\right)$ has a mirror image

$$
\tilde{c}_{0}^{i}=c_{0}^{i} \pm\left(c_{0}^{J}-c_{0}^{I}\right)
$$

whose sign is uniquely determined by the equation

$$
\left|\left[c_{0}^{i}\right]+\left[\tilde{c}_{0}^{i}\right]+2 K^{*}\right| \leq 6 \delta_{0}^{1 / 2} .
$$

Proof. Since $s_{0} \leq 6 l_{0}^{2}$, by $(0.2)$ and the Center Theorem we must have $\mid\left[c_{0}^{I}\right]+$ $\left[c_{0}^{J}\right]+2 K^{*} \mid \leq 2 \delta_{0}^{1 / 2}$. If $\left|\left[c_{0}^{i}\right]-\left[c_{n}^{I}\right]\right| \leq 2 \delta_{0}^{1 / 2}$, we define $\tilde{c}_{0}^{i}=c_{0}^{i}+\left(c_{0}^{J}-c_{0}^{I}\right)$ and it is easy to check that $(0.4)$ holds. Therefore by the Center Theorem we may assume $\left|\left[c_{0}^{i}\right]+\left[c_{0}^{I}\right]+2 K^{*}\right| \leq 2 \delta_{0}^{1 / 2}$. In this case $\tilde{c}_{0}^{i}=c_{0}^{i}-\left(c_{0}^{J}-c_{0}^{I}\right)$ is the required mirror image. 
Remark. Equation (0.4) implies

$$
\left|v\left(\tilde{c}_{0}^{i}\right)-E^{*}\right| \leq \operatorname{cst} \delta_{0}^{1 / 2} \sqrt{E^{*}+1} .
$$

Therefore by Corollary 1, if there is one "close" pair of points in $S_{0}$ then every point in $S_{0}$ has a mirror image that "almost belongs" to $S_{0}$.

Corollary 2 (Spacing Lemma). If $c_{0}^{m} \in S_{0}\left(K^{*}, E^{*}\right)$ for $m=i, j, p$, then two of the three points $c_{0}^{m}$ must be separated by at least $\delta_{0}^{-1 / 6}$.

Proof. If $\left|\left[c_{0}^{i}\right]-\left[c_{0}^{j}\right]\right| \leq 2 \delta_{0}^{1 / 2}$, the proof follows from (0.2). Therefore by the Center Theorem we may assume

$$
\left|\left[c_{0}^{i}\right]+\left[c_{0}^{j}\right]+2 K^{*}\right| \leq 2 \delta_{0}^{1 / 2} \text { and }\left|\left[c_{0}^{i}\right]+\left[c_{0}^{p}\right]+2 K^{*}\right| \leq 2 \delta_{0}^{1 / 2} .
$$

Now we subtract the two inequalities to obtain the result.

Remark. The two corollaries give us a good description of $S_{0}$. There are two possibilities: See Figures 1 and 2.

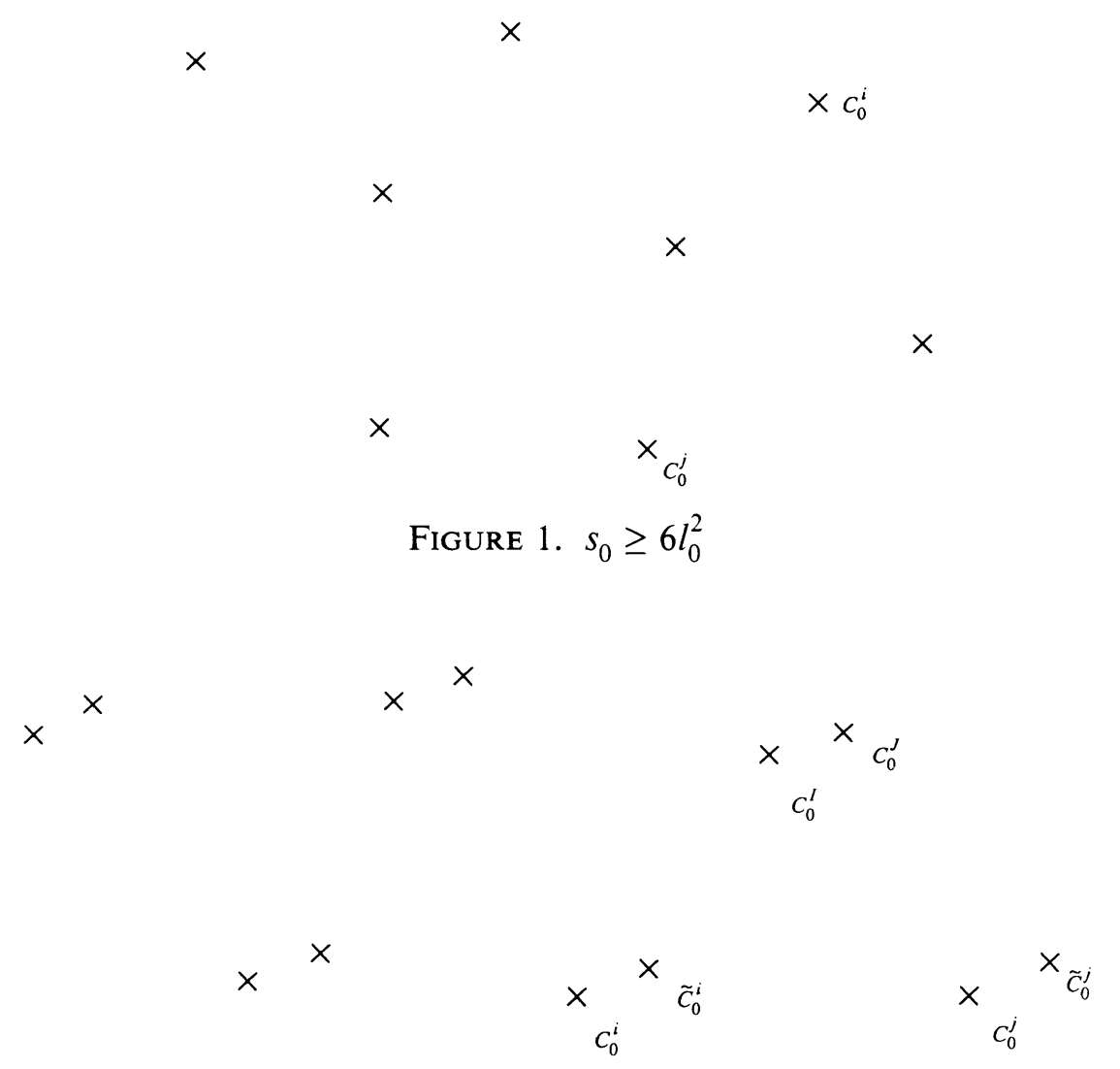

FIGURE 2. $s_{0} \leq 6 l_{0}^{2}$ 


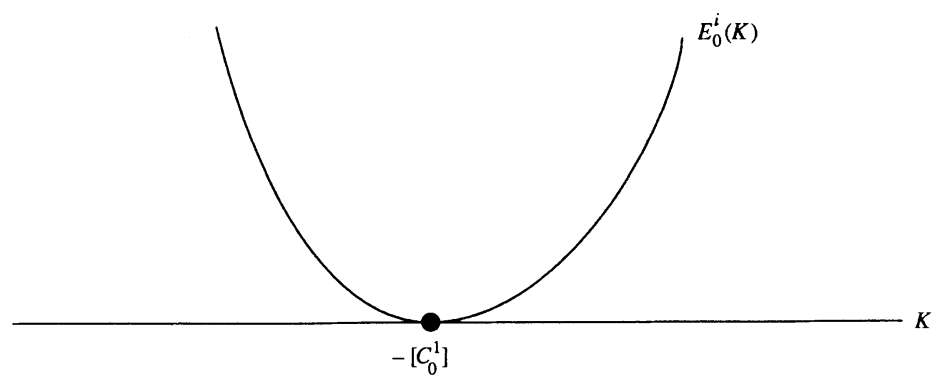

FiguRe 3

We now sketch the proof of the Center Theorem. Since $c_{0}^{r} \in S_{0}\left(K^{*}, E^{*}\right)$ for $r=i, j$, by definition of $S_{0}$ and equation (0.3) we must have

$$
2 \delta_{0} \sqrt{E^{*}+1} \geq\left|E_{0}^{i}\left(K^{*}\right)-E_{0}^{j}\left(K^{*}\right)\right|=\left|E_{0}^{j}\left(K^{*}+\Delta K\right)-E_{0}^{j}\left(K^{*}\right)\right|
$$

where $|\Delta K|=m\left(c_{0}^{i}, c_{0}^{j}\right)$. If we can establish the bound

$$
\left|E_{0}^{j}\left(K^{*}+\Delta K\right)-E_{0}^{j}\left(K^{*}\right)\right| \geq \frac{1}{2} \sqrt{E^{*}+1}|\Delta K|^{2},
$$

it follows that $m\left(c_{0}^{i}, c_{0}^{j}\right)=|\Delta K| \leq 2 \delta_{0}^{1 / 2}$. In $\S 1$ we will study the structure of $E_{0}^{j}(K)$ and establish this bound. Since $E_{0}^{j}(K)$ is a simple quadratic function, it will be easy to understand its structure completely and therefore easy to study the set $S_{0}\left(K^{*}, E^{*}\right)$. See Figure 3.

We end our discussion of $S_{0}\left(K^{*}, E^{*}\right)$ with the following theorem.

Decay Theorem. If $S_{0}\left(K^{*}, E^{*}\right)=\varnothing$ then the matrix elements of $G\left(K^{*}\right) \equiv$ $\left(H\left(K^{*}\right)-E^{*}\right)^{-1}$ decay exponentially fast, i.e.,

$$
\left|\left(H\left(K^{*}\right)-E^{*}\right)^{-1}(x, y)\right| \leq \operatorname{cst} e^{-\gamma_{0}|x-y|} .
$$

Proof. Since $\|\Delta\| \leq 4$ we have

$$
\begin{aligned}
\left(H-E^{*}\right)^{-1} & =\left(\varepsilon \Delta+V-E^{*}\right)^{-1} \\
& =\sum_{n=0}^{\infty}(-1)^{n} \varepsilon^{n}\left[\left(V-E^{*}\right)^{-1} \Delta\right]^{n}\left(V-E^{*}\right)^{-1}
\end{aligned}
$$

converges (if $\varepsilon$ is small compared to $\delta_{0}$ ) and

$$
\begin{aligned}
\left|\left(H-E^{*}\right)^{-1}(x, y)\right| & \leq \frac{\mathrm{cst}}{\delta_{0} \sqrt{E^{*}+1}}\left(\frac{4 \varepsilon}{\delta_{0} \sqrt{E^{*}+1}}\right)^{|x-y|} \\
& \equiv \frac{\mathrm{cst}}{\delta_{0} \sqrt{E^{*}+1}} e^{-\gamma_{0}|x-y|} \cdot \square
\end{aligned}
$$

Remark. If $E^{*} \leq-\frac{1}{2}$ then $\left(H-E^{*}\right)^{-1}$ converges. This follows from $(0.5)$ and the fact that $\left|v_{j}-E^{*}\right| \geq \frac{1}{2}$ for every $j \in \mathbf{Z}^{2}$. Therefore the only interesting 
case is when $E^{*} \geq-\frac{1}{2}$. From now on we will assume that $E^{*}$ is restricted to this set.

Before we define $S_{n}\left(K^{*}, E^{*}\right)$ for every $n$, we shall discuss some perturbation methods that we will frequently use. We will need to restrict our Hamiltonian $H=(\varepsilon \Delta+V)$ to boxes (certain subsets of $\mathbf{Z}^{2}$ ) of increasing size and inductively use information from small boxes to gain information in larger boxes. The smallest boxes will be single sites in $\mathbf{Z}^{2}$.

If $B$ is a region in $\mathrm{Z}^{2}$ we define $H(B)$ to be the operator $H$ restricted to $B$ by defining the matrix elements as follows:

$$
H(B)(i, j)=H(i, j) \text { for } i, j \in B .
$$

We now define the boundary operator $\Gamma_{B}$ by

$$
\Gamma_{B}(i, j)= \begin{cases}\varepsilon & \text { if }|i-j|=1 \text { and }(i \in B, j \notin B) \text { or }(i \notin B, j \in B), \\ 0 & \text { otherwise. }\end{cases}
$$

Note:

$$
H\left(\Gamma_{B}\right) \equiv H-\Gamma_{B}=H(B) \oplus H\left(B^{c}\right) .
$$

We will need to estimate the Green's function $G=(H-E)^{-1}$. To do this we define

$$
G_{\Gamma} \equiv(H(\Gamma)-E)^{-1}=G(B) \oplus G\left(B^{c}\right)
$$

where $G(B) \equiv(H(B)-E)^{-1}, G\left(B^{c}\right) \equiv\left(H\left(B^{c}\right)-E\right)^{-1}$, and $\Gamma=\Gamma_{B}$. The following resolvent identity will be used later:

$$
G=G_{\Gamma}-G_{\Gamma} \Gamma G
$$

We can now define $S_{n+1}\left(K^{*}, E^{*}\right)$ inductively. We assume that $S_{n}\left(K^{*}, E^{*}\right)$ is defined and consists of either

(1) "distant" points $c_{n}^{i}$ for which we define $c_{n+1}^{i} \equiv c_{n}^{i}$ or

(2) "distant" pairs of points $c_{n}^{i}, \tilde{c}_{n}^{i}$ for which we define $c_{n+1}^{i} \equiv \frac{1}{2}\left(c_{n}^{i}+\tilde{c}_{n}^{i}\right)$.

We then put square boxes $I_{n+1}^{i}$ of length $l_{n+1}$ centered at $c_{n+1}^{i}$ where $l_{n+1} \equiv$ $l_{n}^{2}$ for case (1) and $l_{n+1}=l_{n}^{4}$ for case (2). See Figures 4 and 5.

Remark. We must choose our boxes $I_{n+1}^{i}$ so that the boundary of $I_{n+1}^{i}$ does not intersect the boundary of any previous box $I_{m}^{i}(m \leq n)$. An intersection like this would break up the box $I_{m}^{i}$ and cause us to lose information about $\sigma\left(H\left(I_{m}^{i}\right)\right)$.

Definition. We call a box $\Lambda n$ regular if $\partial \Lambda \cap \partial I_{m}^{i}=\varnothing$ and $\partial \Lambda \cap \partial \widetilde{I}_{m}^{i}=\varnothing$ for every $M \leq n$.

Remark. By Appendix D we can always deform our boxes so that they are regular when required. From now on we will assume that all boxes are chosen to be regular. 


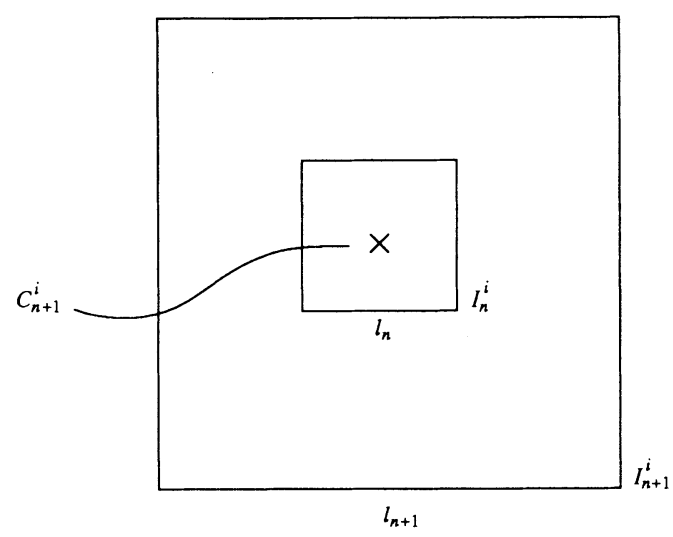

FIGURE 4. $s_{n} \geq 6 l_{n}^{2}$

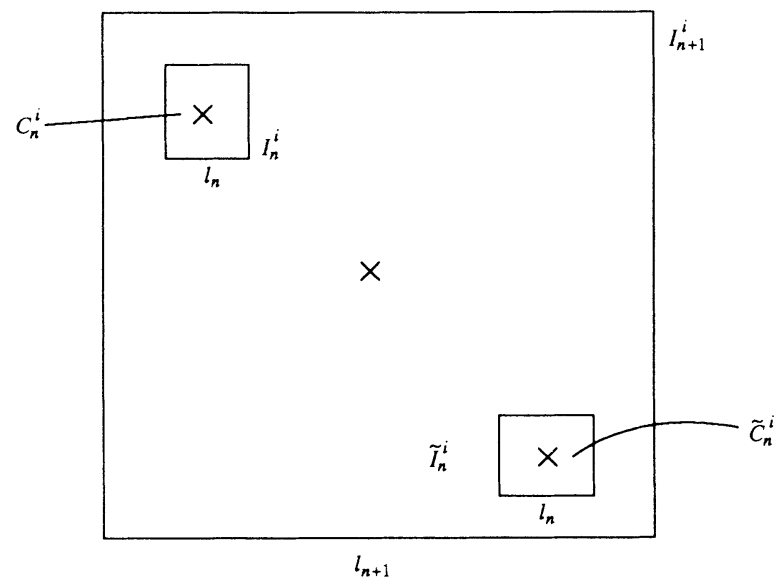

FIGURE 5. $s_{n} \leq 6 l_{n}^{2}$

We say that $c_{n+1}^{i}$ belongs to $S_{n+1}\left(K^{*}, E^{*}\right)$ if $H\left(K^{*}\right)$ restricted to $I_{n+1}^{i}$ has an eigenvalue $E_{n+1}^{i}\left(K^{*}\right)$ such that

$$
\left|E_{n+1}^{i}\left(K^{*}\right)-E^{*}\right| \leq \delta_{n+1} \sqrt{E^{*}+1}
$$

where $\delta_{n+1} \cong \exp \left(-\gamma_{0} l_{n+1}^{2 / 3}\right)$ and $\gamma_{0}$ is the constant defined in the Decay Theorem.

Remark 1. We will show that there are at most two eigenvalues in $\sigma\left(H\left(I_{n+1}^{i}\right)\right)$ that are "near" $E^{*}$. See Lemmas 3.8 and 3.11.

Remark 2. In one-dimensional problems it is known that there are no level crossings; i.e., there is a lower bound on the separation of the eigenvalues in $\sigma\left(H\left(I_{n+1}^{i}\right)\right)$. This is not true in higher dimensions. This will complicate our analysis since we must consider the case when the eigenvalues cross. 
$\S 3$ is devoted to the study of $S_{n}\left(K^{*}, E^{*}\right)$. Here we will list the important results and give a brief sketch of their proofs.

Center Theorem. If $c_{n}^{i}$ and $c_{n}^{j}$ belong to $S_{n}$, then

$$
m\left(c_{n}^{i}, c_{n}^{j}\right) \leq 2 \delta_{n}^{1 / 2} / \delta_{n-1}
$$

where

$$
m\left(c_{n}^{i}, c_{n}^{j}\right) \equiv \min \left(\left|\left[c_{n}^{i}\right]-\left[c_{n}^{j}\right]\right|,\left|\left[c_{n}^{i}\right]+\left[c_{n}^{j}\right]+2 K^{*}\right|\right) .
$$

Corollary 1. Let $s_{n}=\min _{S_{n}}\left|c_{n}^{i}-c_{n}^{j}\right| \equiv\left|c_{n}^{I}-c_{n}^{J}\right|$ and suppose that $s_{n} \leq 6 l_{n}^{2}$. Then every $c_{n}^{i} \in S_{n}$ has a mirror image

$$
\tilde{c}_{n}^{i}=c_{n}^{i} \pm\left(c_{n}^{J}-c_{n}^{I}\right)
$$

whose sign is uniquely determined by the equation

$$
\left|\left[c_{n}^{i}\right]+\left[\tilde{c}_{n}^{i}\right]+2 K^{*}\right| \leq 6 \delta_{n}^{1 / 2} / \delta_{n-1} .
$$

Corollary 2 (Spacing Lemma). If $c_{n}^{m} \in S_{n}$ for $m=i, j, p$ then two of the three points must be separated by at least $\delta_{n}^{-1 / 6}$.

Remark. The proof of these two corollaries goes exactly as it did in the $S_{0}\left(K^{*}, E^{*}\right)$ case after we establish the Center Theorem.

Now we will state a generalized form of the Decay Theorem.

Decay Theorem. If $S_{n} \cap \Lambda=\varnothing$ then

$$
\left|\left(H(\Lambda)-E^{*}\right)^{-1}(x, y)\right| \leq\left(\operatorname{cst} / \delta_{0} \sqrt{E^{*}+1}\right) e^{-\gamma_{n}|x-y|}
$$

provided $|x-y| \geq l_{n}^{5 / 6}$ where $\left(\gamma_{0} / 2\right) \leq \gamma_{n} \leq \gamma_{0}$.

Proof. See Appendix C.

Remark. The Decay Theorem will be the main tool used to prove that eigenfunctions decay exponentially fast.

We now sketch the proof of the Center Theorem. If $c_{n}^{r} \in S_{n}$ for $r=i, j$, there are eigenvalues $E_{n}^{r}\left(K^{*}\right) \in \sigma\left(H\left(I_{n}^{r}\right), K^{*}\right)$ such that $\left|E_{n}^{r}\left(K^{*}\right)-E^{*}\right| \leq$ $\delta_{n} \sqrt{E^{*}+1}$; hence it suffices to show that

$$
\left|E_{n}^{i}\left(K^{*}\right)-E_{n}^{j}\left(K^{*}\right)\right| \geq \frac{1}{2} \delta_{n-1}^{2} \sqrt{E^{*}+1} m\left(c_{n}^{i}, c_{n}^{j}\right)^{2} .
$$

To prove (0.9), we will show that

$$
\sigma\left(H\left(I_{n}^{j}, K^{*}\right)\right)=\sigma\left(H\left(I_{n}^{i}, K^{*}+\Delta K\right)\right)
$$

where $|\Delta K|=m\left(c_{n}^{i}, c_{n}^{j}\right)$ and

$$
\sigma\left(H\left(I_{n}^{i}, K\right)\right)=\sigma\left(H\left(I_{n}^{i},-2\left[c_{n}^{i}\right]-K\right)\right)
$$

for $K$ near $K^{*}$. Equation (0.10) follows from the fact that the box $I_{n}^{j}$ can be thought of as the box $I_{n}^{i}$ translated by the amount $c_{n}^{j}-c_{n}^{i}$. To prove 
(0.11) we note that $v_{y}\left(K_{s}+\delta K\right)=v_{x}\left(K_{s}-\delta K\right)$ for every $x \in I_{n}^{i}$ where $y=2 c_{n}^{i}-x$. Equation $(0.11)$ implies that there is a point $K_{s}=-\left[c_{n}^{i}\right]$ about which the eigenvalues are symmetric.

If we are in the simple case where there is a unique eigenvalue $E_{n}^{i} \in \sigma\left(H\left(I_{n}^{i}\right)\right)$ close to $E^{*}$, then $(0.10)$ implies that

$$
E_{n}^{j}\left(K^{*}\right)=E_{n}^{i}\left(K^{*}+\Delta K\right) .
$$

Therefore the proof of $(0.9)$ reduces to a problem of investigating the derivatives of $E_{n}^{i}(K)$ for $K$ near $K^{*}$. We will use first and second order perturbation theory to calculate the derivatives. The main result is that the first and second derivatives cannot be small simultaneously. This together with the fact that $E_{n}^{i}(K)$ is symmetric yields (0.9). See Figure 6.

If the boxes $I_{n}^{i}$ have two eigenvalues near $E^{*}$, then the calculations described above become more complex. We must analyze both eigenvalues simultaneously in order to calculate the derivatives. (See Appendix B.)

Now we shall explain why $\left|E_{n}^{\prime}\right|$ being small forces $\left|E_{n}^{\prime \prime}\right|$ to be large. This is the key estimate of this paper. The facts we need about the derivatives of the eigenvalue curves will be proved by induction on $n$ as follows. Suppose that $c_{n}^{i} \in S_{n}\left(K^{*}, E^{*}\right)$. Then by the Spacing Lemma there are at most two points $c_{n-1}^{i}, \tilde{c}_{n-1}^{i} \in S_{n-1}\left(K^{*}, E^{*}\right) \cap I_{n}^{i}$ and therefore at most two eigenvalues $E_{n}^{i}\left(K^{*}\right)$, $\mathscr{E}_{n}^{i}\left(K^{*}\right)$ in $\sigma\left(H\left(I_{n}^{i}, K^{*}\right)\right)$ that are near $E^{*}$.

The Decay Theorem will be used to prove that the corresponding eigenfunctions $\psi_{n}^{i}$ and $\Psi_{n}^{i}$ decay exponentially fast outside $I_{n-1}^{i} \cup \widetilde{I}_{n-1}^{i}$. Then using the

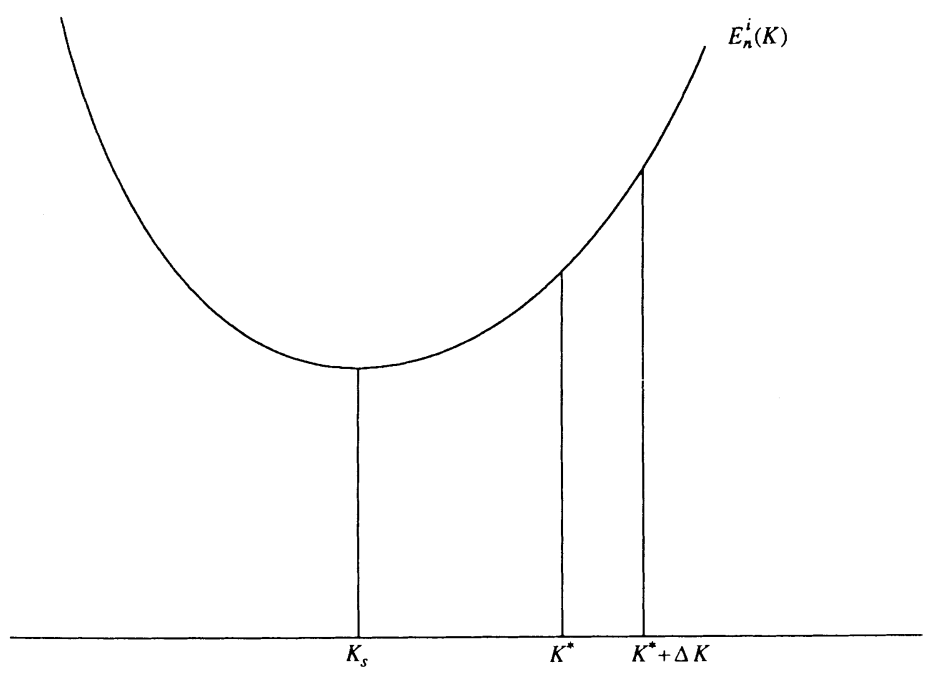

FigURE 6 
fact that these two eigenfunctions are orthonormal we can show that

$$
\begin{gathered}
\psi_{n}^{i} \cong A \psi_{n-1}^{i}+B \tilde{\psi}_{n-1}^{i}, \\
\Psi_{n}^{i} \cong B \psi_{n-1}^{i}-A \tilde{\psi}_{n-1}^{i}
\end{gathered}
$$

where $A^{2}+B^{2} \cong 1$ and $\psi_{n-1}^{i}, \tilde{\psi}_{n-1}^{i}$ are eigenfunctions in the boxes $I_{n-1}^{i}, \widetilde{I}_{n-1}^{i}$ that have eigenvalues $E_{n-1}^{i}, \widetilde{E}_{n-1}^{i}$ near $E^{*}$.

Now we will calculate the derivatives of $E_{n}^{i}$ and relate them to the derivatives of $E_{n-1}^{i}$ and $\widetilde{E}_{n-1}^{i}$. Then by induction we will gain information about $E_{n}^{i}$. By Appendix B we have

$$
\begin{aligned}
\frac{d}{d K} E_{n}^{i} & =\left\langle\psi_{n}^{i}, V^{\prime} \psi_{n}^{i}\right\rangle \\
& \cong A^{2}\left\langle\psi_{n-1}^{i}, V^{\prime} \psi_{n-1}^{i}\right\rangle+B^{2}\left\langle\tilde{\psi}_{n-1}^{i}, V^{\prime} \tilde{\psi}_{n-1}^{i}\right\rangle \\
& =A^{2} \frac{d}{d K} E_{n-1}^{i}+B^{2} \frac{d}{d K} \widetilde{E}_{n-1}^{i} .
\end{aligned}
$$

Then we can use the symmetry of the potential to show that

$$
\frac{d}{d K} \widetilde{E}_{n-1}^{i} \cong-\frac{d}{d K} E_{n-1}^{i}
$$

therefore

$$
\frac{d}{d K} E_{n}^{i} \cong\left(A^{2}-B^{2}\right) \frac{d}{d K} E_{n-1}^{i} .
$$

Again by Appendix B we can show that

$$
\frac{d^{2}}{d K^{2}} E_{n}^{i}=\frac{2\left\langle\psi_{n}^{i}, V^{\prime} \Psi_{n}^{i}\right\rangle^{2}}{E_{n}^{i}-\mathscr{E}_{n}^{i}}+\text { smaller terms }
$$

where

$$
\begin{aligned}
\left\langle\psi_{n}^{i}, V^{\prime} \Psi_{n}^{i}\right\rangle & \cong A B\left\langle\psi_{n-1}^{i}, V^{\prime} \psi_{n-1}^{i}\right\rangle-A B\left\langle\tilde{\psi}_{n-1}^{i}, V^{\prime} \tilde{\psi}_{n-1}^{i}\right\rangle \\
& \cong A B \frac{d}{d K} E_{n-1}^{i}-A B \frac{d}{d K} \widetilde{E}_{n-1}^{i} \\
& \cong 2 A B \frac{d}{d K} E_{n-1}^{i} .
\end{aligned}
$$

Therefore

$$
\frac{d^{2}}{d K^{2}} E_{n}^{i} \cong 2\left(2 A B \frac{d}{d K} E_{n-1}^{i}\right)^{2}\left(E_{n}^{i}-\mathscr{E}_{n}^{i}\right)^{-1}
$$

Since $A^{2}+B^{2} \cong 1$, it is impossible for $A^{2}-B^{2}$ and $A B$ to be small at the same time. By induction we will know that $d E_{n-1}^{i} / d K$ is bounded away from zero; thus by (0.12) and (0.13) it is easy to see that $d E_{n}^{i} / d K$ and $d^{2} E_{n}^{i} / d K^{2}$ cannot be small simultaneously. In particular, we will prove that if

$$
\left|\frac{d}{d K} E_{n}^{i}\right| \leq \delta_{n-1}^{2} \sqrt{E^{*}+1}
$$


then

$$
\left|\frac{d^{2}}{d K^{2}} E_{n}^{i}\right| \geq \frac{1}{2} \sqrt{E^{*}+1} .
$$

Now equation (0.9) follows immediately from this estimate. See Appendix A for more details.

We end our introduction with a brief discussion of the proof of Theorem 2 . To prove the absence of point spectrum we assume that there is a solution to the equation

$$
-\frac{d^{2}}{d x^{2}} \psi+\varepsilon(\cos x+\cos (\alpha x+\vartheta)) \psi=E^{*} \psi .
$$

Let $\phi_{m n}^{K}=e^{i m \vartheta} \hat{\psi}(K+n+m \alpha)$ where $\hat{\psi}$ is the Fourier transform of $\psi$. Then it is easy to show that $\phi^{K}$ is a solution to our lattice equation

$$
H(K) \phi^{K}=(\varepsilon \Delta+V(K)) \phi^{K}=E^{*} \phi^{K} .
$$

In $\S 4$ we will prove the following facts:

(1) For $n$ large enough we must have

$$
S_{n}\left(K, E^{*}\right) \cap \Lambda_{n}=\phi
$$

for almost every $K \in \mathbf{R}$, where $\Lambda_{n}$ are boxes around the origin of length $l_{n}$.

(2) $\phi^{K}$ is polynomially bounded for almost every $K$.

If we assume these facts, we can restrict $(0.14)$ to the boxes $\Lambda_{n}$ and write

$$
H\left(\Lambda_{n}\right) \phi^{K}=E^{*} \phi^{K}+\Gamma\left(\Lambda_{n}\right) \phi^{K},
$$

which implies

$$
\phi^{K}=\left(H\left(\Lambda_{n}\right)-E^{*}\right)^{-1} \Gamma\left(\Lambda_{n}\right) \phi^{K} \equiv G\left(\Lambda_{n}\right) \Gamma\left(\Lambda_{n}\right) \phi^{K} .
$$

The Decay Theorem together with fact (1) implies that the matrix elements $G_{x y}$ decay exponentially fast. Since $\phi^{K}$ is polynomially bounded, it follows that $\phi^{K}(x) \equiv 0$ for every $x$ and almost every $K$. Therefore $\hat{\psi}(K)=0$ for almost every $K$, which implies that $\psi=0$ and therefore not an eigenfunction. This is a contradiction to our assumption.

\section{Definition AND PROPERTIES OF $S_{0}$}

We begin by defining the 0 th singular set

$$
S_{0}\left(K^{*}, E^{*}\right)=\left\{c_{0}^{i} \in \mathbf{Z}^{2}:\left|E_{0}^{i}\left(K^{*}\right)-E^{*}\right| \leq \delta_{0} \sqrt{E^{*}+1}\right\} .
$$

For convenience we have defined

$$
E_{0}^{i}(K)=\left(\left[c_{0}^{i}\right]+K\right)^{2}
$$

to be the value of the potential at $c_{0}^{i}$. In this section we will study the structure of $S_{0}$ and prove the Center Theorem. As stated in the introduction, we will always assume that $E^{*} \geq-\frac{1}{2}$. 
Lemma 1.1. Let $c_{0}^{r} \in S_{0}$ for $r=i, j$; then

(a) $m\left(c_{0}^{i}, c_{0}^{j}\right) \leq \sqrt{2 \delta_{0}\left(E^{*}+1\right)^{1 / 2}}$.

(b) $E_{0}^{i}\left(K^{*}\right)=E_{0}^{j}\left(K^{*}+\Delta K\right)$ where $\Delta K=\left[c_{0}^{i}\right]-\left[c_{0}^{j}\right]$ or $-\left(\left[c_{0}^{i}\right]+\left[c_{0}^{j}\right]+2 K^{*}\right)$ and $|\Delta K|=m\left(c_{0}^{i}, c_{0}^{j}\right)$.

(c) $d E_{0}^{i} / d K \mid \leq 3 \sqrt{E^{*}+1}$ for $\left|K-K^{*}\right| \leq 12 \sqrt{2 \delta_{0}\left(E^{*}+1\right)^{1 / 2}}$.

Proof. By definition of $S_{0}$, we have

$$
\begin{aligned}
2 \delta_{0} \sqrt{E^{*}+1} & \geq\left|E_{0}^{i}\left(K^{*}\right)-E_{0}^{j}\left(K^{*}\right)\right|=\left|\left(\left[c_{0}^{i}\right]+K^{*}\right)^{2}-\left(\left[c_{0}^{j}\right]+K^{*}\right)^{2}\right| \\
& =\left|\left[c_{0}^{i}\right]-\left[c_{0}^{j}\right]\right|\left[c_{0}^{i}\right]+\left[c_{0}^{j}\right]+2 K^{*} \mid,
\end{aligned}
$$

which establishes part (a). The proof of part (b) is obvious from (1.1).

We will now prove part (c). Since $c_{0}^{i} \in S_{0}$, we have

$$
\left|E_{0}^{i}\left(K^{*}\right)-E^{*}\right|=\left|\left(\left[c_{0}^{i}\right]+K^{*}\right)^{2}-E^{*}\right| \leq \delta_{0} \sqrt{E^{*}+1}
$$

which implies $\left|\left[c_{0}^{i}\right]+K^{*}\right| \leq \frac{5}{4} \sqrt{E^{*}+1}$. Now we can estimate

$$
\left|\frac{d}{d K} E_{0}^{i}\right|=2\left|\left[c_{0}^{i}\right]+K\right|=2\left|\left[c_{0}^{i}\right]+K^{*}+K-K^{*}\right| \leq 3 \sqrt{E^{*}+1} .
$$

Remark. Lemma $1.1(\mathrm{a})$ is only a weak version of the Center Theorem since $E^{*}$ may be very large. We will remove the $E^{*}$ dependence and strengthen the lemma.

We note that $E_{0}^{i}(K)=\left(\left[c_{0}^{i}\right]+K\right)^{2}$ is symmetric about $K_{s}=-\left[c_{0}^{i}\right]$. The next lemma tells us that if the first derivative is small, then $K$ is near the symmetry point and the second derivative is big.

Lemma 1.2. If $\left|d E_{0}^{i} / d K\right| \leq \sqrt{E^{*}+1}$ for some $K$ in the interval $\left|K-K^{*}\right| \leq$ $12 \sqrt{2 \delta_{0}\left(E^{*}+1\right)^{1 / 2}}$, then

(a) $\left|d^{2} E_{0}^{i} / d K^{2}\right|=2 \geq \sqrt{E^{*}+1}$ for all $K$.

(b) $\left|d E_{0}^{i} / d K\right| \geq \sqrt{E^{*}+1}\left|K+\left[c_{0}^{i}\right]\right|$.

Proof. The equality in part (a) follows immediately from (1.1). Since $\mid E_{0}^{i}\left(K^{*}\right)-$ $E^{*} \mid \leq \delta_{0} \sqrt{E^{*}+1}$, then

$$
\left(\left[c_{0}^{i}\right]+K^{*}\right)^{2} \geq E^{*}-\delta_{0} \sqrt{E^{*}+1} .
$$

By assumption we have $2\left|\left[c_{0}^{i}\right]+K\right|=\left|d E_{0}^{i} / d K\right| \leq \sqrt{E^{*}+1}$ for some $K$ in the interval $\left|K-K^{*}\right| \leq 12 \sqrt{2 \delta_{0}\left(E^{*}+1\right)^{1 / 2}}$; therefore

$$
\begin{aligned}
\left|\left[c_{0}^{i}\right]+K^{*}\right| & =\left|\left[c_{0}^{i}\right]+K+K^{*}-K\right| \leq \frac{1}{2} \sqrt{E^{*}+1}+12 \sqrt{2 \delta_{0}\left(E^{*}+1\right)^{1 / 2}} \\
& \leq \frac{2}{3} \sqrt{E^{*}+1}
\end{aligned}
$$

If we combine this inequality with the previous inequality, we get

$$
E^{*}-\delta_{0} \sqrt{E^{*}+1} \leq\left(\left[c_{0}^{i}\right]+K^{*}\right)^{2} \leq \frac{4}{9}\left(E^{*}+1\right),
$$

which implies $E^{*} \leq 1$. 
We now prove part (b). By part (a) we can assume $\left|d^{2} E_{0}^{i} / d K^{2}\right| \geq \sqrt{E^{*}+1}$ for all $K$. Therefore,

$$
\left(\frac{d}{d K} E_{0}^{i}\right)(K)=\left(\frac{d}{d K} E_{0}^{i}\right)\left(-\left[c_{0}^{i}\right]\right)+\left(\frac{d^{2}}{d K^{2}} E_{0}^{i}\right)\left(K+\left[c_{0}^{i}\right]\right)
$$

so that

$$
\left|\frac{d}{d K} E_{0}^{i}\right| \geq \sqrt{E^{*}+1}\left|K+\left[c_{0}^{i}\right]\right|
$$

Lemma 1.3. If $c_{0}^{r} \in S_{0}$ for $r=i, j$ then

(a) $2 \delta_{0} \sqrt{E^{*}+1} \geq\left|E_{0}^{i}\left(K^{*}\right)-E_{0}^{j}\left(K^{*}\right)\right| \geq \frac{1}{2} \sqrt{E^{*}+1} m\left(c_{0}^{i}, c_{0}^{j}\right)^{2}$.

(b) (Center Theorem) $m\left(c_{0}^{i}, c_{0}^{j}\right) \leq 2 \delta_{0}^{1 / 2}$.

Proof. The first inequality is obvious since $c_{0}^{r} \in S_{0}$. By Lemma 1.1(b) we can write $E_{0}^{i}\left(K^{*}\right)=E_{0}^{j}\left(K^{*}+\Delta K\right)$ where $|\Delta K|=m\left(c_{0}^{i}, c_{0}^{j}\right)$; hence it suffices to estimate

$$
\left|E_{0}^{j}\left(K^{*}+\Delta K\right)-E_{0}^{j}\left(K^{*}\right)\right| \text {. }
$$

If $\left|d E_{0}^{j} / d K\right| \geq \sqrt{E^{*}+1}$ for all $K$ in the interval $\left|K-K^{*}\right| \leq 12 \sqrt{2 \delta_{0}\left(E^{*}+1\right)^{1 / 2}}$, we are done. If $\left|d E_{0}^{j} / d K\right| \leq \sqrt{E^{*}+1}$ for some $K$ in the interval $\left|K-K^{*}\right| \leq$ $12 \sqrt{2 \delta_{0}\left(E^{*}+1\right)^{1 / 2}}$, Lemma 1.2(a) implies that $\left|d^{2} E_{0}^{j} / d K^{2}\right| \geq \sqrt{E^{*}+1}$ for all $K$. Since $E_{0}^{j}(K)$ is symmetric about $K_{s}=-\left[c_{0}^{j}\right]$, we may assume $K_{s} \leq K^{*} \leq$ $K^{*}+\Delta K$. Therefore

$$
\begin{aligned}
\left|E_{0}^{j}\left(K^{*}+\Delta K\right)-E_{0}^{j}\left(K^{*}\right)\right| & =E_{0}^{j}\left(K^{*}+\Delta K\right)-E_{0}^{j}\left(K^{*}\right) \\
& =\frac{d}{d K} E_{0}^{j}\left(K^{*}\right) \Delta K+\frac{1}{2}\left(\frac{d^{2}}{d K^{2}} E_{0}^{j}\right)(\Delta K)^{2} \\
& \geq \frac{1}{2} \sqrt{E^{*}+1} m\left(c_{0}^{i}, c_{0}^{j}\right)^{2} .
\end{aligned}
$$

The Center Theorem follows immediately from part (a).

Remark. We have removed the $E^{*}$ dependence from Lemma 1.1(a).

Lemma 1.4 (Decay Theorem). If $S_{0} \cap \Lambda=\varnothing$ then

$$
\left|(H(\Lambda)-E)^{-1}(x, y)\right| \leq \frac{\mathrm{cst}}{\delta_{0} \sqrt{E^{*}+1}} e^{-\gamma_{0}|x-y|}
$$

for $\left|E-E^{*}\right| \leq\left(\delta_{0} / 5\right) \sqrt{E^{*}+1}$ and $\left|K-K^{*}\right| \leq 4 \delta_{0}^{3 / 2}$ where

Proof. See (0.5).

$$
\gamma_{0}=\ln \left(\left(\delta_{0} \sqrt{E^{*}+1}\right) / 4 \varepsilon\right) \text {. }
$$

Before we consider the next singular set $S_{1}$, we group together nearby elements of $S_{0}$. There are two cases to consider, depending on the size of $s_{0}=\min _{s_{0}}\left|c_{0}^{i}-c_{0}^{j}\right|$. If $s_{0} \geq 6 l_{0}^{2}$, we construct boxes $I_{1}^{i}$ centered at $c_{1}^{i} \equiv c_{0}^{i}$. We 
choose the length of the box to be $l_{1} \equiv l_{0}^{2}$. If $s_{0} \leq 6 l_{0}^{2}$, by (0.4) each $c_{0}^{i} \in S_{0}$ has a mirror image $\tilde{c}_{0}^{i}$ which satisfies

$$
\left|\left[\tilde{c}_{0}^{i}\right]+\left[c_{0}^{i}\right]+2 K^{*}\right| \leq 6 \delta_{0}^{1 / 2} .
$$

In this case we construct boxes $I_{1}^{i}$ centered at $c_{1}^{i} \equiv \frac{1}{2}\left(c_{0}^{i}+\tilde{c}_{0}^{i}\right)$. We choose the length of the box to be $l_{1} \equiv l_{0}^{4}$. Finally we define $\bar{S}_{0}=\left\{c_{1}^{i}\right\}$. (See Figures 4 and 5 of the introduction.)

\section{Definition AND PROPERTIES OF $S_{1}$}

In this section we shall define $S_{1}$ and prove the Center Theorem for $S_{1}$.

$$
S_{1}\left(K^{*}, E^{*}\right) \equiv\left\{c_{1}^{i} \in \bar{S}_{0}: \operatorname{dist}\left(\sigma\left(H\left(I_{1}^{i}\right)\right), E^{*}\right) \leq \delta_{1} \sqrt{E^{*}+1}\right\} .
$$

We will consider the two cases described at the end of $\S 1$ separately.

Case 1. $s_{0} \geq 6 l_{0}^{2}$.

Lemma 2.1. If $c_{1}^{r} \in S_{1}$ for $r=i, j$, then $m\left(c_{1}^{i}, c_{1}^{j}\right) \leq \delta_{0}^{3 / 2}$.

Proof. Since $c_{1}^{r}=c_{0}^{r}$, by Lemma $1.3(\mathrm{a})$ it suffices to show that $\mid E_{0}^{i}\left(K^{*}\right)-$ $E_{0}^{j}\left(K^{*}\right) \mid=O\left(\delta_{0}^{4} \sqrt{E^{*}+1}\right)$. By definition of $S_{1}$, there are eigenvalues $E_{1}^{r}\left(K^{*}\right) \in$ $\sigma\left(H\left(I_{1}^{r}\right)\right)$ such that $\left|E_{1}^{r}\left(K^{*}\right)-E^{*}\right| \leq \delta_{1} \sqrt{E^{*}+1}$. Let $\psi_{1}^{r}$ be the corresponding wave functions. By choosing $\varepsilon \leq \delta_{0}^{4}$ we have

$$
\left\|\left(V\left(I_{1}^{r}\right)-E^{*}\right) \psi_{1}^{r}\right\|=\left\|\left(-\varepsilon \Delta+E_{1}^{r}\left(K^{*}\right)-E^{*}\right) \psi_{1}^{r}\right\| \leq \operatorname{cst} \delta_{0}^{4} \sqrt{E^{*}+1} ;
$$

hence $\psi_{1}^{r}$ serves as a good trial wave function and we conclude that

$$
\left|E_{0}^{r}\left(K^{*}\right)-E^{*}\right| \leq \operatorname{cst} \delta_{0}^{4} \sqrt{E^{*}+1} .
$$

Remark. With a finer analysis we will show that Lemma 2.1 continues to hold if $\delta_{0}^{3 / 2}$ is replaced by $2 \delta_{1}^{1 / 2} / \delta_{0}$.

By definition of $S_{1}$, there exists an eigenvalue $E_{1}^{i}\left(K^{*}\right) \in \sigma\left(H\left(I_{1}^{i}, K^{*}\right)\right)$ which is near $E^{*}$. We will show that we can extend $E_{1}^{i}$ as a function of $K$ for $K$ near $K^{*}$.

Lemma 2.2. If $c_{1}^{i} \in S_{1}$ then

(a) There exists a unique eigenvalue $E_{1}^{i}(K) \in \sigma\left(H\left(I_{1}^{i}\right)\right)$ such that

$$
\left|E_{1}^{i}(K)-E^{*}\right| \leq \operatorname{cst} \delta_{0}^{3 / 2} \sqrt{E^{*}+1}
$$

for every $K$ in the interval $\left|K-K^{*}\right| \leq 2 \delta_{0}^{3 / 2}$.

(b) The corresponding wave function $\psi_{1}^{i}$ decays rapidly away from $c_{1}^{i}$; i.e., $\left|\psi_{1}^{i}(x)\right| \leq \operatorname{cst} e^{-\gamma_{0}\left|x-c_{1}^{i}\right|}$.

(c) Any other eigenvalue $\mathscr{E}_{1}^{i} \in \sigma\left(H\left(I_{1}^{i}\right)\right)$ must obey $\left|\mathscr{E}_{1}^{i}-E^{*}\right| \geq\left(\delta_{0} / 5\right) \sqrt{E^{*}+1}$. 
(d) $E_{1}^{j}\left(K^{*}\right)=E_{1}^{i}\left(K^{*}+\Delta K\right)$ where $\Delta K=\left[c_{1}^{j}\right]-\left[c_{1}^{i}\right]$ or $-\left(\left[c_{1}^{i}\right]+\left[c_{1}^{j}\right]+2 K^{*}\right)$ and $|\Delta K|=m\left(c_{1}^{i}, c_{1}^{j}\right)$.

Proof. By (2.1) and Lemma 1.1(c) we have

$$
\left|E_{0}^{i}(K)-E^{*}\right| \leq \operatorname{cst} \delta_{0}^{3 / 2} \sqrt{E^{*}+1}
$$

for $\left|K-K^{*}\right| \leq 2 \delta_{0}^{3 / 2}$. Now,

$$
\begin{aligned}
\left\|\left(H\left(I_{1}^{i}\right)-E^{*}\right) \delta\left(x-c_{1}^{i}\right)\right\| & =\left\|\left(\varepsilon \Delta+V\left(I_{1}^{i}\right)-E^{*}\right) \delta\left(x-c_{1}^{i}\right)\right\| \\
& \leq \operatorname{cst} \delta_{0}^{3 / 2} \sqrt{E^{*}+1},
\end{aligned}
$$

which implies the existence of $E_{1}^{i}(K) \in \sigma\left(H\left(I_{1}^{i}\right)\right)$ such that

$$
\left|E_{1}^{i}(K)-E^{*}\right| \leq \operatorname{cst} \delta_{0}^{3 / 2} \sqrt{E^{*}+1} \text { for }\left|K-K^{*}\right| \leq 2 \delta_{0}^{3 / 2}
$$

To prove part (b) we use the defining equation $H\left(I_{1}^{i}\right) \psi_{1}^{i}=E_{1}^{i} \psi_{1}^{i}$ and rewrite it as $\left(\bar{H}-E_{1}^{i}\right) \psi_{1}^{i}=R \psi_{1}^{i}$. The matrix $\bar{H}$ is the same as $H\left(I_{1}^{i}\right)$ except that the potential at $c_{1}^{i}$ is changed to make $S_{0}$ empty and $\left(\bar{H}-E_{1}^{i}\right)^{-1}$ decay (see Lemma 1.4). We note that $R=\bar{H}-H\left(I_{1}^{i}\right)$ has one nonzero element in the place corresponding to $c_{1}^{i}$. Thus we can express $\psi_{1}^{i}=\left(\bar{H}-E_{1}^{i}\right)^{-1} R \psi_{1}^{i}$, which implies $\left|\psi_{1}^{i}(x)\right| \leq \operatorname{cst} e^{-\gamma_{0}\left|x-c_{1}^{i}\right|}$.

To establish part (c) we assume that there is another eigenvalue $\mathscr{E}_{1}^{i}$ that obeys $\left|\mathscr{E}_{1}^{i}-E^{*}\right| \leq\left(\delta_{0} / 5\right) \sqrt{E^{*}+1}$. Then the same argument shows that its wave function $\Psi_{1}^{i}$ decays away from $c_{1}^{i}$. Since the wave functions $\psi_{1}^{i}$ and $\Psi_{1}^{i}$ are orthogonal, we have a contradiction.

The proof of part (d) follows from (0.10) and the uniqueness of $E_{1}^{j}$.

Lemma 2.3. If $K$ belongs to the interval $\left|K-K^{*}\right| \leq 2 \delta_{0}^{3 / 2}$, then $d E_{1}^{i} / d K=$ $d E_{0}^{i} / d K+O\left(\delta_{0}^{2} \sqrt{E^{*}+1}\right)$.

Proof. By Appendix B, $d E_{1}^{i} / d K=\left\langle\psi_{1}^{i}, V^{\prime} \psi_{1}^{i}\right\rangle$ and $d E_{0}^{i} / d K=\left\langle\psi_{0}^{i}, V^{\prime} \psi_{0}^{i}\right\rangle$ where $\psi_{0}^{i}(x)=\delta\left(x-c_{1}^{i}\right)$. Thus to prove the lemma we need only show that

$$
\left\|\psi_{1}^{i}-\psi_{0}^{i}\right\| \leq \operatorname{cst} \delta_{0}^{3} \quad \text { and } \quad\left\|V^{\prime}\right\| \leq \delta_{0}^{-1} \sqrt{E^{*}+1} .
$$

The first inequality is a consequence of Lemma 2.2(b), so it remains to estimate

$$
\begin{aligned}
\left\|V^{\prime}\right\| & \leq \max _{I_{1}}\left|v_{x}^{\prime}\right|=\max 2|[x]+K| \\
& \leq \max 2\left|[x]-\left[c_{1}^{i}\right]\right|+\max 2\left|\left[c_{1}^{i}\right]+K\right| \\
& \leq \operatorname{cst} l_{1}+\max \left|\frac{d}{d K} E_{0}^{i}\right|,
\end{aligned}
$$

which by Lemma $1.1(\mathrm{c})$ is bounded by

$$
\operatorname{cst} l_{1}+3 \sqrt{E^{*}+1} \leq \operatorname{cst} l_{1} \sqrt{E^{*}+1} \leq \delta_{0}^{-1} \sqrt{E^{*}+1} .
$$


Lemma 2.4. If $c_{1}^{i} \in S_{1}$ then

(a) $\left|d E_{1}^{i} / d K\right| \leq\left(3+\delta_{0}\right) \sqrt{E^{*}+1}$ for every $K$ in the interval $\left|K-K^{*}\right| \leq 2 \delta_{0}^{3 / 2}$.

(b) If $\left|d E_{1}^{i} / d K\right| \leq \delta_{0}^{2} \sqrt{E^{*}+1}$ for some $K$ in the interval $\left|K-K^{*}\right| \leq 2 \delta_{0}^{3 / 2}$ then

$$
\left|\frac{d^{2}}{d K^{2}} E_{1}^{i}\right| \geq\left(1-\delta_{0}\right) \sqrt{E^{*}+1} ;
$$

moreover $d^{2} E_{1}^{i} / d K^{2}$ has a unique sign.

Proof. We use Lemmas 2.3 and 1.1(c) to establish part (a).

We now prove part (b). If the hypothesis holds, then by Lemma 2.3 we must have

$$
\left|\frac{d}{d K} E_{0}^{i}\right| \leq \operatorname{cst} \delta_{0}^{2} \sqrt{E^{*}+1} .
$$

Lemma $1.2\left(\right.$ a) implies that $2 \geq \sqrt{E^{*}+1}$; therefore we can use Appendix B to write

$$
\frac{d^{2}}{d K^{2}} E_{1}^{i}=2+2\left\langle V^{\prime} \psi_{1}^{i},\left(E_{1}^{i}-H\right)_{\perp}^{-1} V^{\prime} \psi_{1}^{i}\right\rangle .
$$

We will show that the remainder term is $O\left(\delta_{0}^{3} \sqrt{E^{*}+1}\right)$. Taking norms, the remainder term is bounded by $2\left\|V^{\prime} \psi_{1}^{i}\right\|^{2}\left\|\left(E_{1}^{i}-H\right)_{\perp}^{-1}\right\|$. By Lemma 2.2(c) we have $\left\|\left(E_{1}^{i}-H\right)_{\perp}^{-1}\right\|=O\left(\delta_{0} \sqrt{E^{*}+1}\right)^{-1}$, so it remains to estimate $\left\|V^{\prime} \psi_{1}^{i}\right\|^{2}$. Using (2.2) we get

$$
\begin{aligned}
\left\|V^{\prime} \psi_{1}^{i}\right\|^{2} & \leq\left(\left\|V^{\prime}\left(\psi_{1}^{i}-\psi_{0}^{i}\right)\right\|+\left\|V^{\prime} \psi_{0}^{i}\right\|\right)^{2} \\
& \leq\left(\left\|V^{\prime}\right\| \delta_{0}^{3}+\left|\frac{d}{d K} E_{0}^{i}\right|\right)^{2} \\
& \leq\left(\delta_{0}^{-1} \sqrt{E^{*}+1} \delta_{0}^{3}+\left|\frac{d}{d K} E_{0}^{i}\right|\right)^{2}
\end{aligned}
$$

which by $(2.3)$ is bounded by $\operatorname{cst}\left(\delta_{0}^{2} \sqrt{E^{*}+1}\right)^{2}$.

By $(0.11)$ we know that there is a point $K_{s}=-\left[c_{1}^{i}\right]$ about which $E_{1}^{i}(K)$ is symmetric. It is not clear that the symmetry point belongs to the interval of definition of $E_{1}^{i}(K)$. The next lemma tells us that if $d E_{1}^{i} / d K$ is small then $K$ must be near the symmetry point.

Lemma 2.5. If $\left|d E_{1}^{i} / d K\right| \leq \delta_{0}^{2} \sqrt{E^{*}+1}$ for some $K$ in the interval $\left|K-K^{*}\right| \leq$ $\delta_{0}^{3 / 2}$, then $\left|d E_{1}^{i} / d K\right| \geq \frac{1}{2} \sqrt{E^{*}+1}\left|K+\left[c_{1}^{i}\right]\right|$.

Proof. If the hypotheses holds then by Lemma 2.3 we have

$$
\left|d E_{0}^{i} / d K\right| \leq \operatorname{cst} \delta_{0}^{2} \sqrt{E^{*}+1}
$$

and by Lemma $1.2(\mathrm{~b})$ we get $\left|K+\left[c_{1}^{i}\right]\right|=O\left(\delta_{0}^{2}\right)$, which implies $\left|K^{*}+\left[c_{1}^{i}\right]\right| \leq$ $2 \delta_{0}^{3 / 2}$. Therefore the symmetry point $K_{s}=-\left[c_{1}^{i}\right]$ lies in the interval $\left|K-K^{*}\right| \leq$ 
$2 \delta_{0}^{3 / 2}$, which is the interval of definition of $E_{1}^{i}(K)$. We now use Lemma 2.4(b) and Appendix A to complete the proof.

Lemma 2.6. If $c_{1}^{r} \in S_{1}$ for $r=i, j$ then

(a) $2 \delta_{1} \sqrt{E^{*}+1} \geq\left|E_{1}^{i}\left(K^{*}\right)-E_{1}^{j}\left(K^{*}\right)\right| \geq \frac{1}{2} \delta_{0}^{2} \sqrt{E^{*}+1} m\left(c_{1}^{i}, c_{1}^{j}\right)^{2}$.

(b) (Center Theorem) $m\left(c_{1}^{i}, c_{1}^{j}\right) \leq 2 \delta_{1}^{1 / 2} / \delta_{0}$.

Proof. The first inequality in part (a) holds since $c_{1}^{r} \in S_{1}$. By Lemma 2.2(d) we have

$$
E_{1}^{i}\left(K^{*}\right)-E_{1}^{j}\left(K^{*}\right)=E_{1}^{i}\left(K^{*}\right)-E_{1}^{i}\left(K^{*}+\Delta K\right) .
$$

If $\left|d E_{1}^{i} / d K\right| \geq \delta_{0}^{2} \sqrt{E^{*}+1}$ for $\left|K-K^{*}\right| \leq \delta_{0}^{3 / 2}$ then part (a) follows immediately. Therefore we may assume $\left|d E_{1}^{i} / d K\right| \leq \delta_{0}^{2} \sqrt{E^{*}+1}$ for some $\left|K-K^{*}\right| \leq$ $\delta_{0}^{3 / 2}$. Then by Lemma 2.5 , the symmetry point belongs to the interval of definition of $E_{1}^{i}(K)$. With the definition of $\Delta K$ from Lemma 2.2(d) we use Lemma 2.4(b) and Appendix A to establish part (a). The Center Theorem follows immediately from part (a).

Remark 1. Lemma 2.6(b) strengthens Lemma 2.1.

Remark 2. We are now finished with case $1\left(s_{0} \geq 6 l_{0}^{2}\right)$.

Case 2. $s_{0} \leq 6 l_{0}^{2}$.

We would like to include the symmetry point $K_{s}=-\left[c_{1}^{i}\right]$ in our interval of $K$ 's. The next lemma tells us how wide an interval we need to take in order to do this.

Lemma 2.7. If $c_{1}^{r} \in S_{1}$ for $r=i, j$ then

(a) $\left|K^{*}+\left[c_{1}^{r}\right]\right| \leq 3 \delta_{0}^{1 / 2}$.

(b) $\max \left(\left|\left[c_{1}^{i}\right]-\left[c_{1}^{j}\right]\right|,\left|\left[c_{1}^{i}\right]+\left[c_{1}^{j}\right]+2 K^{*}\right|\right) \leq 6 \delta_{0}^{1 / 2}$.

Proof. We use (1.2) and the definition of $c_{1}^{i}$ to establish part (a). Part (b) follows immediately from part (a).

Since $c_{0}^{i} \in S_{0}$, we have $\left|E_{0}^{i}\left(K^{*}\right)-E^{*}\right| \leq \delta_{0} \sqrt{E^{*}+1}$. For convenience we define $\widetilde{E}_{0}^{i}(K)$ to be the value of the potential at $\tilde{c}_{0}^{i}$; therefore

$$
\widetilde{E}_{0}^{i}(K)=\left(\left[\tilde{c}_{0}^{i}\right]+K\right)^{2}=E_{0}^{i}\left(-K-\left[\tilde{c}_{0}^{i}\right]-\left[c_{0}^{i}\right]\right) .
$$

With equation (2.4) we can transfer information from $E_{0}^{i}$ to $\widetilde{E}_{0}^{i}$; in particular, Lemma 1.1(c) implies that $\left|\widetilde{E}_{0}^{i}\left(K^{*}\right)-E^{*}\right| \leq \operatorname{cst} \delta_{0}^{1 / 2} \sqrt{E^{*}+1}$. Thus in each box $I_{1}^{i}$ we have two values of the potential near $E^{*}$ which will be used to generate two eigenvalues in $\sigma\left(H\left(I_{1}^{i}\right)\right)$ near $E^{*}$.

Lemma 2.8. If $c_{1}^{r} \in S_{1}$ for $r=i, j$ then

(a) There exist two eigenvalues $E_{1}^{i}(K)$ and $\mathscr{E}_{1}^{i}(K) \in \sigma\left(H\left(I_{1}^{i}\right)\right)$ such that

$$
\left|E_{1}^{i}(K)-E^{*}\right| \leq \operatorname{cst} \delta_{0}^{1 / 2} \sqrt{E^{*}+1}
$$

for every $K$ in the interval $\left|K-K^{*}\right| \leq 6 \delta_{0}^{1 / 2}$. The same holds for $\mathscr{E}_{1}^{i}(K)$. 
(b) The corresponding wave functions $\psi_{1}^{i}$ and $\Psi_{1}^{i}$ decay rapidly away from $c_{0}^{i}$ and $\tilde{c}_{0}^{i}$, i.e.,

$$
\left|\psi_{1}^{i}(x)\right| \leq \operatorname{cst}\left(e^{-\gamma_{0}\left|x-c_{0}^{i}\right|}+e^{-\gamma_{0}\left|x-\tilde{c}_{0}^{i}\right|} .\right.
$$

The same holds for $\Psi_{1}^{i}$.

(c) If $\widehat{E}$ is any other eigenvalue in $\sigma\left(H\left(I_{1}^{i}\right)\right)$ then

$$
\left|\widehat{E}-E^{*}\right| \geq \delta_{0}^{1 / 8} \sqrt{E^{*}+1} .
$$

(d) $E_{1}^{j}\left(K^{*}\right)=E_{1}^{i}\left(K^{*}+\Delta K\right)$ or $E_{1}^{j}\left(K^{*}\right)=\mathscr{E}_{1}^{i}\left(K^{*}+\Delta K\right)$ where $\Delta K=\left[c_{1}^{j}\right]-\left[c_{1}^{i}\right]$ and $|\Delta K| \leq 6 \delta_{0}^{1 / 2}$.

Remark. We assume that the eigenvalues $E_{1}^{i}(K)$ and $\mathscr{E}_{1}^{i}(K)$ are labeled so that they are differentiable functions of $K$. See Appendix B for details.

Proof. We already know that

$$
\left|E_{0}^{i}\left(K^{*}\right)-E^{*}\right| \leq \operatorname{cst} \delta_{0}^{1 / 2} \sqrt{E^{*}+1} .
$$

By Lemma 1.1(c) we can extend this for $K$ near $K^{*}$, i.e.,

$$
\left|E_{0}^{i}(K)-E^{*}\right| \leq \operatorname{cst} \delta_{0}^{1 / 2} \sqrt{E^{*}+1}
$$

for $\left|K-K^{*}\right| \leq 6 \delta_{0}^{1 / 2}$. We now use $\delta\left(x-c_{0}^{i}\right)$ as a trial wave function for $H\left(I_{1}^{i}\right)$, giving us

$$
\left\|\left(H\left(I_{1}^{i}\right)-E^{*}\right) \delta\left(x-c_{0}^{i}\right)\right\| \leq \operatorname{cst} \delta_{0}^{1 / 2} \sqrt{E^{*}+1},
$$

which implies the existence of $E_{1}^{i}(K) \in \sigma\left(H\left(I_{1}^{i}\right)\right)$ such that

$$
\left|E_{1}^{i}(K)-E^{*}\right| \leq \operatorname{cst} \delta_{0}^{1 / 2} \sqrt{E^{*}+1} .
$$

The same analysis with $E_{0}^{i}(K)$ replaced by $\widetilde{E}_{0}^{i}(K)$ gives us the second eigenvalue $\mathscr{E}_{1}^{i}(K) \in \sigma\left(H\left(I_{1}^{i}\right)\right)$.

To establish the decay of the wave function we note that $\left|v_{j}(K)-E^{*}\right| \geq$ $2 \delta_{0}^{1 / 8} \sqrt{E^{*}+1}$ for every $j \in I_{1}^{i} \backslash\left\{c_{0}^{i}, \tilde{c}_{0}^{i}\right\}$ and every $\left|K-K^{*}\right| \leq 6 \delta_{0}^{1 / 2}$, or else there would be three points $j, c_{0}^{i}, \tilde{c}_{0}^{i}$ belonging to $S_{0}\left(K, E^{*}\right) \cap I_{1}^{i}$ (with $\delta_{0}$ replaced by $\left.2 \delta_{0}^{1 / 8}\right)$. This is impossible by the Spacing Lemma. Therefore

$$
\left|v_{j}(K)-E_{1}^{i}(K)\right| \geq \delta_{0}^{1 / 8} \sqrt{E^{*}+1} \text { for } j \in I_{1}^{i} \backslash\left\{c_{0}^{i}, \tilde{c}_{0}^{i}\right\} .
$$

We write the defining equation $H\left(I_{1}^{i}\right) \psi_{1}^{i}=E_{1}^{i} \psi_{1}^{i}$ as $\left(\bar{H}-E_{1}^{i}\right) \psi_{1}^{i}=R \psi_{1}^{i}$ where $\bar{H}$ is the same as $H\left(I_{1}^{i}\right)$ except the potential is changed at $c_{0}^{i}$ and $\tilde{c}_{0}^{i}$ to make $\left(\bar{H}-E_{1}^{i}\right)^{-1}$ decay. Now we can express $\psi_{1}^{i}=\left(\bar{H}-E_{1}^{i}\right)^{-1} R \psi_{1}^{i}$, which gives us

$$
\left|\psi_{1}^{i}(x)\right| \leq \operatorname{cst}\left(e^{-\gamma_{0}\left|x-c_{0}^{i}\right|}+e^{-\gamma_{0}\left|x-\tilde{c}_{0}^{i}\right|}\right) .
$$

To prove part (c) we assume that there is another eigenvalue $\widehat{E} \in \sigma\left(H\left(I_{1}^{i}\right)\right)$ such that $\left|\widehat{E}-E^{*}\right| \leq \delta_{0}^{1 / 8} \sqrt{E^{*}+1}$. Then the same argument shows that its 
wave function decays away from $c_{0}^{i}$ and $\tilde{c}_{0}^{i}$, thus violating orthogonality. The proof of part (d) follows from (0.10) and Lemma 2.7(b).

Since we have two eigenvalues in $\sigma\left(H\left(I_{1}^{i}\right)\right)$ which are close to $E^{*}$, the 0 th order eigenvalues $E_{0}^{i}$ and $\widetilde{E}_{0}^{i}$ have some special properties.

Lemma 2.9. If $K$ is in the interval $\left|K-K^{*}\right| \leq 6 \delta_{0}^{1 / 2}$, then

(a) $\left|d E_{0}^{i} / d K+d \widetilde{E}_{0}^{i} / d K\right| \leq \operatorname{cst} \delta_{0}^{1 / 2}$.

(b) $\left|d E_{0}^{i} / d K\right| \geq \delta_{0}^{1 / 8} \sqrt{E^{*}+1}$.

Proof. By (2.4) we have

$$
\begin{aligned}
\left|\frac{d}{d K} E_{0}^{i}+\frac{d}{d K} \widetilde{E}_{0}^{i}\right| & =\left|\left(\frac{d}{d K} E_{0}^{i}\right)(K)-\left(\frac{d}{d K} E_{0}^{i}\right)\left(-K-\left[c_{0}^{i}\right]-\left[\tilde{c}_{0}^{i}\right]\right)\right| \\
& \leq \max \left|\frac{d^{2}}{d K^{2}} E_{0}^{i}\right|\left|2 K+\left[c_{0}^{i}\right]+\left[\tilde{c}_{0}^{i}\right]\right| \\
& =2\left|2 K+\left[c_{0}^{i}\right]+\left[\tilde{c}_{0}^{i}\right]\right|,
\end{aligned}
$$

which by (1.2) is bounded by $\operatorname{cst} \delta_{0}^{1 / 2}$.

We now prove part (b). If $\left|d E_{0}^{i} / d K\right| \leq \delta_{0}^{1 / 8} \sqrt{E^{*}+1}$ for some $K$, then by Lemma 1.2(b) we have $\left|d E_{0}^{i} / d K\right| \geq \sqrt{E^{*}+1}\left|K+\left[c_{0}^{i}\right]\right|$. Now

$$
\left|K+\left[c_{0}^{i}\right]\right| \geq\left|\left[c_{0}^{i}\right]-\left[c_{1}^{i}\right]\right|-\left|K-K^{*}\right|-\left|K^{*}+\left[c_{1}^{i}\right]\right|,
$$

which by Lemma $2.7(\mathrm{a})$ is greater than

$$
\left|\left[c_{0}^{i}\right]-\left[c_{1}^{i}\right]\right|-\operatorname{cst} \delta_{0}^{1 / 2} .
$$

We note that

$$
\left|\left[c_{0}^{i}\right]-\left[c_{1}^{i}\right]\right|=\left|\left[c_{0}^{i}\right]-\frac{\left[c_{0}^{i}\right]+\left[\tilde{c}_{0}^{i}\right]}{2}\right|=\frac{1}{2}\left|\left[c_{0}^{i}\right]-\left[\tilde{c}_{0}^{i}\right]\right|,
$$

which by $(0.2)$ is bounded below by cst $/\left|c_{0}^{i}-\tilde{c}_{0}^{i}\right|^{2} \geq \mathrm{cst} / l_{0}^{4}$. Therefore

$$
\left|d E_{0}^{i} / d K\right| \geq\left(\operatorname{cst} / l_{0}^{4}-\operatorname{cst} \delta_{0}^{1 / 2}\right) \sqrt{E^{*}+1} \geq \delta_{0}^{1 / 8} \sqrt{E^{*}+1},
$$

giving us a contradiction.

Lemma 2.10. If $c_{1}^{i} \in S_{1}$ then

$$
\begin{aligned}
& \psi_{1}^{i}(x)=A \delta\left(x-c_{0}^{i}\right)+B \delta\left(x-\tilde{c}_{0}^{i}\right)+R(x), \\
& \Psi_{1}^{i}(x)=B \delta\left(x-c_{0}^{i}\right)-A \delta\left(x-\tilde{c}_{0}^{i}\right)+S(x),
\end{aligned}
$$

where $\|R\| \leq \delta_{0},\|S\| \leq \delta_{0}$, and $1 \geq A^{2}+B^{2} \geq 1-\delta_{0}$.

Proof. It is obvious that we can express

$$
\psi_{1}^{i}(x)=A \delta\left(x-c_{0}^{i}\right)+B \delta\left(x-\tilde{c}_{0}^{i}\right)+R(x) .
$$


Lemma 2.8(b) gives us $\|R\| \leq \delta_{0}^{2}$, and since $\psi$ is normalized we have $1 \geq$ $A^{2}+B^{2} \geq 1-O\left(\delta_{0}^{2}\right)$. We can express $\Psi_{1}^{i}$ in a similar manner. Since $\psi_{1}^{i}$ and $\Psi_{1}^{i}$ are orthogonal, we must have

$$
\Psi_{1}^{i}(x)=B \delta\left(x-c_{0}^{i}\right)-A \delta\left(x-\tilde{c}_{0}^{i}\right)+S(x) .
$$

Lemma 2.11. If $c_{1}^{i} \in S_{1}$ then

(a)

(b)

$$
\begin{aligned}
\frac{d}{d K} E_{1}^{i} & =\left(A^{2}-B^{2}\right) \frac{d}{d K} E_{0}^{i}+O\left(\delta_{0}^{1 / 2} \sqrt{E^{*}+1}\right), \\
\frac{d}{d K} \mathscr{E}_{1}^{i} & =\left(B^{2}-A^{2}\right) \frac{d}{d K} E_{0}^{i}+O\left(\delta_{0}^{1 / 2} \sqrt{E^{*}+1}\right) .
\end{aligned}
$$

$$
\begin{aligned}
& \frac{d^{2}}{d K^{2}} E_{1}^{i}=\frac{2\left\langle\psi_{1}^{i}, V^{\prime} \Psi_{1}^{i}\right\rangle^{2}}{E_{1}^{i}-\mathscr{E}_{1}^{i}}+O\left(\delta_{0}^{-1 / 8} \sqrt{E^{*}+1}\right), \\
& \frac{d^{2}}{d K^{2}} \mathscr{E}_{1}^{i}=\frac{2\left\langle\psi_{1}^{i}, V^{\prime} \Psi_{1}^{i}\right\rangle^{2}}{\mathscr{E}_{1}^{i}-E_{1}^{i}}+O\left(\delta_{0}^{-1 / 8} \sqrt{E^{*}+1}\right)
\end{aligned}
$$

(c)

(both hold where $E_{1}^{i} \neq \mathscr{E}_{1}^{i}$ ).

(d)

$$
\left\langle\psi_{1}^{i}, V^{\prime} \Psi_{1}^{i}\right\rangle=2 A B \frac{d}{d K} E_{0}^{i}+O\left(\delta_{0}^{1 / 2} \sqrt{E^{*}+1}\right) .
$$

$$
\left|\frac{d}{d K} E_{1}^{i}+\frac{d}{d K} \mathscr{E}_{1}^{i}\right| \leq \operatorname{cst} \delta_{0}^{1 / 2} \sqrt{E^{*}+1} .
$$

Proof. By Lemma 2.8(b) we have

$$
\begin{aligned}
\frac{d}{d K} E_{1}^{i} & =\left\langle\psi_{1}^{i}, V^{\prime} \psi_{1}^{i}\right\rangle=A^{2} \frac{d}{d K} E_{0}^{i}+B^{2} \frac{d}{d K} \widetilde{E}_{0}^{i}+O\left(\delta_{0}^{2} \sqrt{E^{*}+1}\right) \\
& =\left(A^{2}-B^{2}\right) \frac{d}{d K} E_{0}^{i}+B^{2}\left(\frac{d}{d K} E_{0}^{i}+\frac{d}{d K} \widetilde{E}_{0}^{i}\right)+O\left(\delta_{0}^{2} \sqrt{E^{*}+1}\right) .
\end{aligned}
$$

We use Lemma 2.9(a) to bound the second term of the last line and complete the proof of part (a).

To prove part (b) we use Appendix B to write

$$
\frac{d^{2}}{d K^{2}} E_{1}^{i}=2+2 \frac{\left\langle\psi_{1}^{i}, V^{\prime} \Psi_{1}^{i}\right\rangle^{2}}{E_{1}^{i}-\mathscr{E}_{1}^{i}}+2\left\langle V^{\prime} \psi_{1}^{i},\left(E_{1}^{i}-H\right)_{\perp \perp}^{-1} V^{\prime} \psi_{1}^{i}\right\rangle
$$

The remainder term is bounded by $2\left\|V^{\prime} \psi_{1}^{i}\right\|^{2}\left\|\left(E_{1}^{i}-H\right)_{\perp \perp}^{-1}\right\|$, and by Lemma 2.8(c) we have $\left\|\left(E_{1}^{i}-H\right)_{\perp \perp}^{-1}\right\| \leq\left(\delta_{0}^{1 / 8} \sqrt{E^{*}+1}\right)^{-1}$. It remains to estimate

$$
\left\|V^{\prime} \psi_{1}^{i}\right\|^{2}=A^{2}\left(\frac{d}{d K} E_{0}^{i}\right)^{2}+B^{2}\left(\frac{d}{d K} \widetilde{E}_{0}^{i}\right)^{2}+O\left(\delta_{0}^{2} \sqrt{E^{*}+1}\right)^{2} .
$$

We now use Lemma 1.1(c) to bound the first two terms of the last equation. Therefore $\left\|V^{\prime} \psi_{1}^{i}\right\|^{2} \leq \operatorname{cst}\left(E^{*}+1\right)$, and part (b) is established. 
We now prove part (c). By Lemma 2.9(a) we can estimate

$$
\begin{aligned}
\left\langle\psi_{1}^{i}, V^{\prime} \Psi_{1}^{i}\right\rangle & =A B \frac{d}{d K} E_{0}^{i}-A B \frac{d}{d K} \widetilde{E}_{0}^{i}+O\left(\delta_{0}^{2} \sqrt{E^{*}+1}\right) \\
& =2 A B \frac{d}{d K} E_{0}^{i}+O\left(\delta_{0}^{1 / 2} \sqrt{E^{*}+1}\right) .
\end{aligned}
$$

Part (d) follows immediately from parts (a) and (b).

Lemma 2.12. If $\left|d E_{1}^{i} / d K\right| \leq \delta_{0}^{1 / 4} \sqrt{E^{*}+1}$ for some $K$ in the interval $\left|K-K^{*}\right| \leq$ $6 \delta_{0}^{1 / 2}$ then

(a) $\left|\left\langle\psi_{1}^{i}, V^{\prime} \Psi_{1}^{i}\right\rangle\right| \geq \frac{1}{3} \delta_{0}^{1 / 8} \sqrt{E^{*}+1}$.

(b) $\left|d^{2} E_{1}^{i} / d K^{2}\right| \geq \sqrt{E^{*}+1}$ at all points where $E_{1}^{i} \neq \mathscr{E}_{1}^{i} ;$ moreover $d^{2} E_{1}^{i} / d K^{2}$ has a unique sign.

Remark 1. Lemma 2.12 holds if $E_{1}^{i}$ is replaced by $\mathscr{E}_{1}^{i}$.

Remark 2. We will show later that under the hypothesis of Lemma 2.12, $E_{1}^{i} \neq$ $\mathscr{E}_{1}^{i}$ for $\left|K-K^{*}\right| \leq 6 \delta_{0}^{1 / 2}$.

Proof. If $\left|d E_{1}^{i} / d K\right| \leq \delta_{0}^{1 / 4} \sqrt{E^{*}+1}$ then by Lemma 2.11 (a) we have

$$
\left|A^{2}-B^{2}\right|\left|d E_{0}^{i} / d K\right| \leq \operatorname{cst} \delta_{0}^{1 / 4} \sqrt{E^{*}+1}
$$

which by Lemma $2.9(\mathrm{~b})$ implies $\left|A^{2}-B^{2}\right|=O\left(\delta_{0}^{1 / 8}\right)$. Since $1 \geq A^{2}+B^{2} \geq 1-\delta_{0}$ we must have $|A B| \geq \frac{1}{4}$. Lemmas $2.11(\mathrm{c})$ and 2.9 (b) give us

$$
\mid\left\langle\psi_{1}^{i}, V^{\prime} \Psi_{1}^{i}\right\rangle \geq \frac{1}{3} \delta_{0}^{1 / 8} \sqrt{E^{*}+1} .
$$

To prove part (b) we use Lemmas 2.11(b), 2.8(a) and part (a).

By (0.11) we have

$$
E_{1}^{i}\left(K_{s}+\delta K\right)=E_{1}^{i}\left(K_{s}-\delta K\right) \text { or } E_{1}^{i}\left(K_{s}+\delta K\right)=\mathscr{E}_{1}^{i}\left(K_{s}-\delta K\right) .
$$

Each eigenvalue curve itself might not be symmetric about $K_{s}$, but the union of both curves is symmetric. (See Figure 2.)

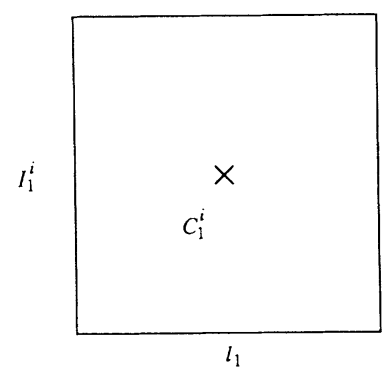

$\left(s_{0} \geq 6 l_{0}^{2}\right)$

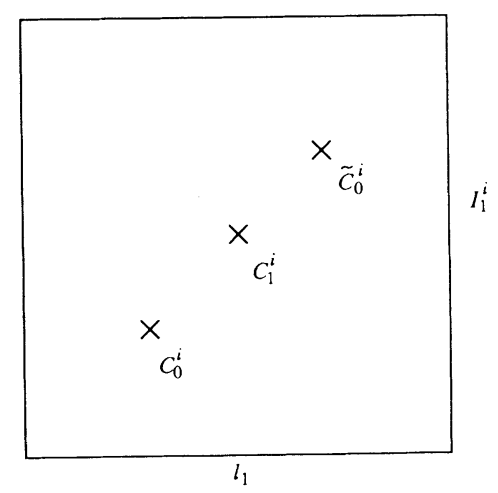

$\left(s_{0} \leq 6 l_{0}^{2}\right)$

FIGURE 1 


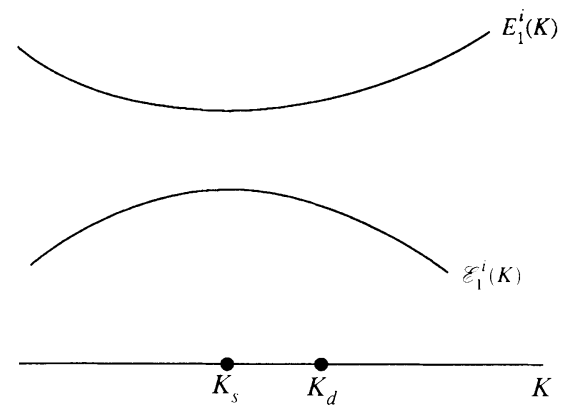

$E_{1}^{i}\left(K_{s}\right)>\mathscr{E}_{1}^{i}\left(K_{s}\right)$

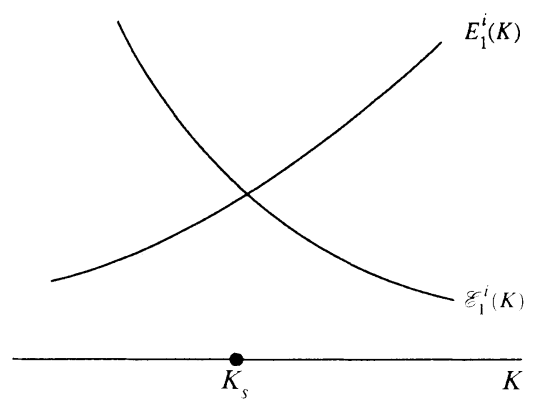

$E_{1}^{i}\left(K_{s}\right)=\mathscr{E}_{1}^{i}\left(K_{s}\right)$

Figure 2
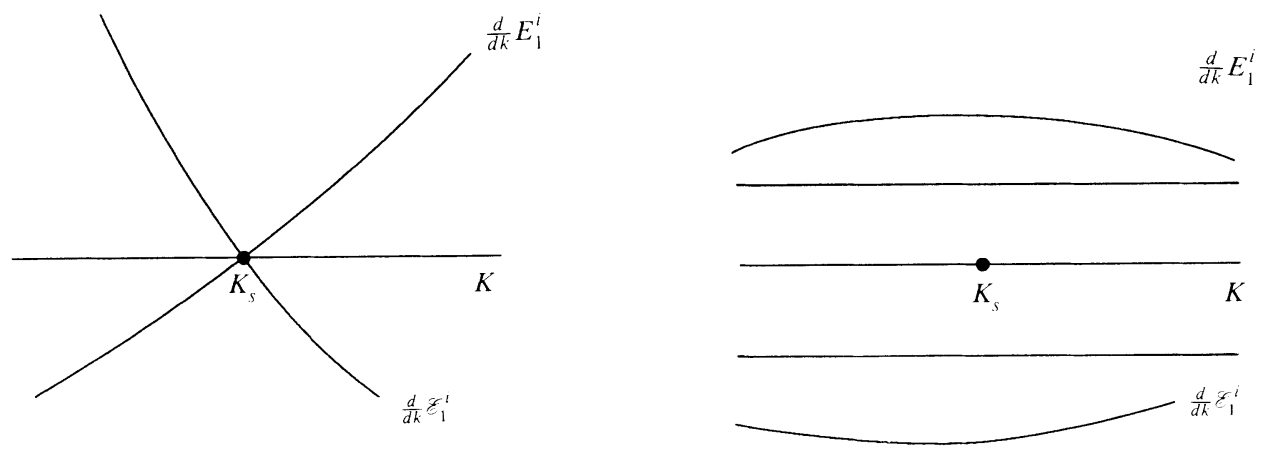

$E_{1}^{i}\left(K_{s}\right)>\mathscr{F}_{1}^{i}\left(K_{s}\right)$

$E_{1}^{i}\left(K_{s}\right)=r_{1}^{i}\left(K_{s}\right)$

Figure 3

Lemma 2.13. If $c_{1}^{i} \in S_{1}$ then

$$
\left|E_{1}^{i}\left(K_{2}\right)-E_{1}^{i}\left(K_{1}\right)\right| \geq \delta_{0}^{2} \sqrt{E^{*}+1} \min \left\{\begin{array}{l}
\left|K_{2}-K_{1}\right|^{2}, \\
\left|K_{2}+K_{1}+2\left[c_{1}^{i}\right]\right|^{2}
\end{array}\right.
$$

for any points $K_{1}, K_{2}$ belonging to the interval $\left|K-K^{*}\right| \leq 6 \delta_{0}^{1 / 2}$. The same is true for $\mathscr{E}_{1}^{i}$.

Proof. There are two cases to consider:

Case I. $E_{1}^{i}\left(K_{s}\right) \neq \mathscr{E}_{1}^{i}\left(K_{s}\right)$ (see Figures 2, 3).

Without loss of generality we may assume $E_{1}^{i}\left(K_{s}\right)>\mathscr{E}_{1}^{i}\left(K_{s}\right)$. By (2.5) we must have

$$
E_{1}^{i}\left(K_{s}+\delta K\right)=E_{1}^{i}\left(K_{s}-\delta K\right) \quad \text { and } \quad \mathscr{E}_{1}^{i}\left(K_{s}+\delta K\right)=\mathscr{E}_{1}^{i}\left(K_{s}-\delta K\right)
$$

for $\delta K$ small; therefore

$$
\left(d E_{1}^{i} / d K\right)\left(K_{s}\right)=\left(d \mathscr{E}_{1}^{i} / d K\right)\left(K_{s}\right)=0 .
$$


By Lemmas 2.12(a) and 2.11(b) we see that $d^{2} E_{1}^{i} / d K^{2}$ and $d^{2} \mathscr{E}_{1}^{i} / d K^{2}$ are large with opposite signs. Appendix A and Lemma 2.12(b) give us

$$
\left|E_{1}^{i}\left(K_{2}\right)-E_{1}^{i}\left(K_{1}\right)\right| \geq \delta_{0}^{2} \sqrt{E^{*}+1} \min \left\{\begin{array}{l}
\left|K_{2}-K_{1}\right|^{2}, \\
\left|K_{2}+K_{1}+2\left[c_{1}^{i}\right]\right|^{2} .
\end{array}\right.
$$

Case II. $E_{1}^{i}\left(K_{s}\right)=\mathscr{E}_{1}^{i}\left(K_{s}\right)$ (see Figures 2,3$)$.

In this case we will show that

$$
\left|\frac{d}{d K} E_{1}^{i}\right| \geq \delta_{0}^{1 / 4} \sqrt{E^{*}+1} \text { and }\left|\frac{d}{d K} \mathscr{E}_{1}^{i}\right| \geq \delta_{0}^{1 / 4} \sqrt{E^{*}+1}
$$

holds for every $K$ in the interval $\left|K-K^{*}\right| \leq 6 \delta_{0}^{1 / 2}$; moreover the derivatives have opposite signs.

If (2.7) holds, the lemma follows immediately. We must now prove (2.7). We start by showing that $(2.7)$ is true at the symmetry point $K_{s}$. Differentiating the equation $H\left(I_{1}^{i}\right) \psi_{1}^{i}=E_{1}^{i} \psi_{1}^{i}$ yields

$$
H^{\prime} \psi_{1}^{i}+H\left(\frac{d}{d K} \psi_{1}^{i}\right)=\left(\frac{d}{d K} E_{1}^{i}\right) \psi_{1}^{i}+E_{1}^{i}\left(\frac{d}{d K} \psi_{1}^{i}\right) .
$$

Note that $H^{\prime}=V^{\prime}$ and then set $K=K_{s}$. Let $P$ be the projection onto the nullspace of $(H-E)$ where $E=E_{1}^{i}\left(K_{s}\right)=\mathscr{E}_{1}^{i}\left(K_{s}\right)$. Now multiply the last equation by $P$. We have

$$
P V^{\prime} \psi_{1}^{i}+P H\left(\frac{d}{d K} \psi_{1}^{i}\right)=\left(\frac{d}{d K} E_{1}^{i}\right) P \psi_{1}^{i}+E_{1}^{i} P\left(\frac{d}{d K} \psi_{1}^{i}\right) .
$$

Notice that the second term on the left, $P H\left(d \psi_{1}^{i} / d K\right)=H P\left(d \psi_{1}^{i} / d K\right)=$ $E_{1}^{i} P\left(d \psi_{1}^{i} / d K\right)$, is canceled by the same term on the right; therefore $P V^{\prime} \psi_{1}^{i}=$ $\left(d E_{1}^{i} / d K\right) P \psi_{1}^{i}$. Using the fact that $P \psi_{1}^{i}=\psi_{1}^{i}$, we rewrite this equation as $\left(P V^{\prime} P\right) \psi_{1}^{i}=\left(d E_{1}^{i} / d K\right)\left(K_{s}\right) \psi_{1}^{i}$, which says that $\left(d E_{1}^{i} / d K\right)\left(K_{s}\right)$ is an eigenvalue of the $2 \times 2$ matrix $P V^{\prime} P$. The same argument with $E_{1}^{i}$ replaced by $\mathscr{E}_{1}^{i}$ implies that $\left(d \mathscr{E}_{1}^{i} / d K\right)\left(K_{s}\right)$ is the other eigenvalue.

To calculate these eigenvalues we will represent $P V^{\prime} P$ in a special basis. To do this we define the operator $S$ by

$$
(S \phi)(x)=\phi\left(2 c_{1}^{i}-x\right) .
$$

The eigenfunctions of $S$ are $\psi_{s}$ and $\psi_{a}$, symmetric and antisymmetric wave functions, i.e.,

$$
\psi_{s}\left(2 c_{1}^{i}-x\right)=\psi_{s}(x) \text { and } \psi_{a}\left(2 c_{1}^{i}-x\right)=-\psi_{a}(x)
$$

Note that $H\left(I_{1}^{i}, K_{s}\right)$ commutes with $S$, which allows us to express $P V^{\prime} P$ in the basis $\left\{\psi_{s}, \psi_{a}\right\}$. In this basis we have

$$
P V^{\prime} P=\left(\begin{array}{cc}
\left\langle\psi_{s}, V^{\prime} \psi_{s}\right\rangle & \left\langle\psi_{s}, V^{\prime} \psi_{a}\right\rangle \\
\left\langle\psi_{s}, V^{\prime} \psi_{a}\right\rangle & \left\langle\psi_{a}, V^{\prime} \psi_{a}\right\rangle
\end{array}\right) \quad\left(\text { at } K=K_{s}\right) .
$$


Now by the symmetry properties of $\psi_{s}, \psi_{a}$, and $V^{\prime}\left(K_{s}\right)$ we have $\left\langle\psi_{s}, V^{\prime} \psi_{s}\right\rangle=$ $\left\langle\psi_{a}, V^{\prime} \psi_{a}\right\rangle=0$, which gives us

$$
P V^{\prime} P=\left(\begin{array}{cc}
0 & \left\langle\psi_{s}, V^{\prime} \psi_{a}\right\rangle \\
\left\langle\psi_{s}, V^{\prime} \psi_{a}\right\rangle & 0
\end{array}\right)
$$

and therefore

$$
\left(\frac{d}{d K} E_{1}^{i}\right)\left(K_{s}\right)=-\left(\frac{d}{d K} \mathscr{E}_{1}^{i}\right)\left(K_{s}\right)=\left\langle\psi_{s}, V^{\prime} \psi_{a}\right\rangle .
$$

We must show that $\left(d E_{1}^{i} / d K\right)\left(K_{s}\right)$ is not too small and then extend this for $\left|K-K^{*}\right| \leq 6 \delta_{0}^{1 / 2}$. Using the symmetry properties and the decay of the wave function, we have

$$
\left(\frac{d}{d K} E_{1}^{i}\right)\left(K_{s}\right)=2 \psi_{s}\left(c_{0}^{i}\right) \psi_{a}\left(c_{0}^{i}\right)\left(\frac{d}{d K} E_{0}^{i}\right)\left(K_{s}\right)+O\left(\delta_{0}^{2} \sqrt{E^{*}+1}\right)
$$

where $\left|\psi_{s}\left(c_{0}^{i}\right)\right| \cong 1 / \sqrt{2}$ and $\left|\psi_{a}\left(c_{0}^{i}\right)\right| \cong 1 / \sqrt{2}$. Lemma $2.9(\mathrm{~b})$ together with $(2.8)$ gives us

$$
\left|\left(d E_{1}^{i} / d K\right)\left(K_{s}\right)\right| \geq \delta_{0}^{1 / 4} \sqrt{E^{*}+1} .
$$

We must show that this continues to hold for all $K$ in the interval $\mid K-$ $K^{*} \mid \leq 6 \delta_{0}^{1 / 2}$. To see this, we plot the derivatives of $E_{1}^{i}(K)$ and $\mathscr{E}_{1}^{i}(K)$. We claim that these curves cannot enter the dashed region. (See Figure 3.) If $\left|d E_{1}^{i} / d K\right| \leq \delta_{0}^{1 / 4} \sqrt{E^{*}+1}$ for some $K$, by Lemmas 2.12(b) and 2.11(b) we have $d^{2} E_{1}^{i} / d K^{2}>0$. This contradicts Figure 3 .

Lemma 2.14. Let $c_{1}^{i} \in S_{1}$; then

(a) $\left|d E_{1}^{i} / d K\right| \leq\left(3+\delta_{0}^{1 / 4}\right) \sqrt{E^{*}+1}$.

(b) If $\left|d E_{1}^{i} / d K\right| \leq \delta_{0}^{2} \sqrt{E^{*}+1}$ for some $K$ in the interval $\left|K-K^{*}\right| \leq 6 \delta_{0}^{1 / 2}$ then $\left|d E_{1}^{i} / d K\right| \geq \frac{1}{2} \sqrt{E^{*}+1}\left|K+\left[c_{1}^{i}\right]\right|$.

Remark. The same is true for $\mathscr{E}_{1}^{i}$.

Proof. We use Lemmas 2.11(a) and 1.1(c) to prove part (a). To establish part (b) we use Appendix A and Lemma 2.12(b).

Lemma 2.15. If $c_{1}^{r} \in S_{1}$ for $r=i, j$ then

(a) $\left|E_{1}^{i}\left(K^{*}\right)-E_{1}^{j}\left(K^{*}\right)\right| \geq \delta_{0}^{2} \sqrt{E^{*}+1} m\left(c_{1}^{i}, c_{1}^{j}\right)^{2}$. The same is true for $\mid \mathscr{E}_{1}^{i}\left(K^{*}\right)$ $-\mathscr{E}_{1}^{j}\left(K^{*}\right) \mid$.

(b) $\left|E_{1}^{i}\left(K^{*}\right)-\mathscr{E}_{1}^{j}\left(K^{*}\right)\right| \geq \delta_{0}^{2} \sqrt{E^{*}+1} m\left(c_{1}^{i}, c_{1}^{j}\right)^{2}$.

(c) (Center Theorem) $m\left(c_{1}^{i}, c_{1}^{j}\right) \leq 2 \delta_{1}^{1 / 2} / \delta_{0}$.

Proof. If $E_{1}^{j}\left(K^{*}\right)=E_{1}^{i}\left(K^{*}+\Delta K\right)$, we must estimate $\left|E_{1}^{i}\left(K^{*}\right)-E_{1}^{i}\left(K^{*}+\Delta K\right)\right|$. By Lemma 2.13, this difference is bounded below by

$$
\delta_{0}^{2} \sqrt{E^{*}+1} \min \left\{\begin{array}{l}
|\Delta K|^{2} \\
\left|2 K^{*}+\Delta K+2\left[c_{1}^{i}\right]\right|^{2} .
\end{array}\right.
$$


We now use the definition of $\Delta K$ in Lemma 2.8(d) to obtain part (a).

We must consider the case where $E_{1}^{j}\left(K^{*}\right)=\mathscr{E}_{1}^{i}\left(K^{*}+\Delta K\right)$ and estimate $\left|E_{1}^{i}\left(K^{*}\right)-\mathscr{E}_{1}^{i}\left(K^{*}+\Delta K\right)\right|$. A careful inspection of the geometry of the eigenvalue curves gives us

$$
\left|E_{1}^{i}\left(K^{*}\right)-\mathscr{E}_{1}^{i}\left(K^{*}+\Delta K\right)\right| \geq \min \left\{\begin{array}{l}
\left|E_{1}^{i}\left(K^{*}\right)-E_{1}^{i}\left(K^{*}+\Delta K\right)\right|, \\
\left|\mathscr{E}_{1}^{i}\left(K^{*}\right)-\mathscr{E}_{1}^{i}\left(K^{*}+\Delta K\right)\right| .
\end{array}\right.
$$

We now use the result in the first part of the proof to establish parts (a) and (b).

To prove the Center Theorem, we note that by definition of $S_{1}$, one of the eigenvalue differences in parts (a) and (b) must be bounded above by $2 \delta_{1} \sqrt{E^{*}+1}$.

It is important to know that the eigenvalues $E_{1}^{i}(K)$ and $\mathscr{E}_{1}^{i}(K)$ may cross only at the symmetry point $K_{s}=-\left[c_{1}^{i}\right]$, and their separation grows as $K$ moves away from $K_{s}$.

Lemma 2.16. If $c_{1}^{i} \in S_{1}$ then

$$
\left|E_{1}^{i}(K)-\mathscr{E}_{1}^{i}(K)\right| \geq \delta_{0}^{2} \sqrt{E^{*}+1}\left|K+\left[c_{1}^{i}\right]\right|
$$

for all $K$ in the interval $\left|K-K^{*}\right| \leq 6 \delta_{0}^{1 / 2}$.

Proof. We must consider two cases:

Case I. $E_{1}^{i}\left(K_{s}\right)=\mathscr{E}_{1}^{i}\left(K_{s}\right)$ (see Figures 2, 3).

In this case, by (2.7) we have

$$
\begin{aligned}
\left|\left(E_{1}^{i}-\mathscr{E}_{1}^{i}\right)(K)\right| & =\left|\left(E_{1}^{i}-\mathscr{E}_{1}^{i}\right)\left(K_{s}\right)+\frac{d}{d K}\left(E_{1}^{i}-\mathscr{E}_{1}^{i}\right)(\widehat{K})\left(K-K_{s}\right)\right| \\
& \geq 2 \delta_{0}^{1 / 4} \sqrt{E^{*}+1}\left|K-K_{s}\right| \\
& =2 \delta_{0}^{1 / 4} \sqrt{E^{*}+1}\left|K+\left[c_{1}^{i}\right]\right| .
\end{aligned}
$$

Case II. $E_{1}^{i}\left(K_{s}\right)>\mathscr{E}_{1}^{i}\left(K_{s}\right)$ (see Figures 2, 3).

By (2.6) and Lemma 2.12(a) we have

$$
\left|\left\langle\psi_{1}^{i}, V^{\prime} \Psi_{1}^{i}\right\rangle\left(K_{s}\right)\right| \geq \frac{1}{3} \delta_{0}^{1 / 8} \sqrt{E^{*}+1} ;
$$

therefore there must be an interval $K_{s} \leq K \leq K_{d}$ where $\left|\left\langle\psi_{1}^{i}, V^{\prime} \Psi_{1}^{i}\right\rangle(K)\right| \geq$ $\frac{1}{3} \delta_{0}^{1 / 8} \sqrt{E^{*}+1}$. If $K$ is in this interval then

$$
\begin{aligned}
\left(E_{1}^{i}-\mathscr{E}_{1}^{i}\right)(K)= & \left(E_{1}^{i}-\mathscr{E}_{1}^{i}\right)\left(K_{s}\right)+\frac{d}{d K}\left(E_{1}^{i}-\mathscr{E}_{1}^{i}\right)\left(K_{s}\right)\left(K-K_{s}\right) \\
& +\frac{1}{2} \frac{d^{2}}{d K^{2}}\left(E_{1}^{i}-\mathscr{E}_{1}^{i}\right)(\widehat{K})\left(K-K_{s}\right)^{2} .
\end{aligned}
$$


By Lemma 2.11(b) we have

$$
\begin{aligned}
\frac{d^{2}}{d K^{2}}\left(E_{1}^{i}-\mathscr{E}_{1}^{i}\right)(\widehat{K}) & =\frac{4\left\langle\psi_{1}^{i}, V^{\prime} \Psi_{1}^{i}\right\rangle^{2}}{\left(E_{1}^{i}-\mathscr{E}_{1}^{i}\right)(\widehat{K})}+O\left(\delta_{0}^{-1 / 8} \sqrt{E^{*}+1}\right) \\
& \geq \operatorname{cst} \frac{\left(\delta_{0}^{1 / 8} \sqrt{E^{*}+1}\right)^{2}}{\left(E_{1}^{i}-\mathscr{E}_{1}^{i}\right)(K)}
\end{aligned}
$$

which implies

$$
\left(E_{1}^{i}-\mathscr{E}_{1}^{i}\right)(K) \geq \operatorname{cst} \frac{\left(\delta_{0}^{1 / 8} \sqrt{E^{*}+1}\right)^{2}}{\left(E_{1}^{i}-\mathscr{E}_{1}^{i}\right)(K)}\left(K-K_{s}\right)^{2}
$$

and proves the lemma.

We must now consider the case when $K \geq K_{d}$. By definition of $K_{d}$ we have

$$
\left|\left\langle\psi_{1}^{i}, V^{\prime} \Psi_{1}^{i}\right\rangle\right| \leq \frac{1}{3} \delta_{0}^{1 / 8} \sqrt{E^{*}+1} \text { for } K \geq K_{d} ;
$$

therefore by Lemma $2.12(\mathrm{a})$ we must have

$$
\frac{d}{d K} E_{1}^{i} \geq \delta_{0}^{1 / 4} \sqrt{E^{*}+1} \text { and } \frac{d}{d K} \mathscr{E}_{1}^{i} \leq-\delta_{0}^{1 / 4} \sqrt{E^{*}+1}
$$

giving us

$$
\begin{aligned}
\left(E_{1}^{i}-\mathscr{E}_{1}^{i}\right)(K) & =\left(E_{1}^{i}-\mathscr{E}_{1}^{i}\right)\left(K_{d}\right)+\frac{d}{d K}\left(E_{1}^{i}-\mathscr{E}_{1}^{i}\right)(\widehat{K})\left(K-K_{d}\right) \\
& \geq\left(E_{1}^{i}-\mathscr{E}_{1}^{i}\right)\left(K_{d}\right)+2 \delta_{0}^{1 / 4} \sqrt{E^{*}+1}\left(K-K_{d}\right) .
\end{aligned}
$$

Now by the first part of the proof we can bound

$$
\left(E_{1}^{i}-\mathscr{E}_{1}^{i}\right)\left(K_{d}\right) \geq \operatorname{cst} \delta_{0}^{1 / 8} \sqrt{E^{*}+1}\left(K_{d}-K_{s}\right)
$$

which gives us

$$
\left(E_{1}^{i}-\mathscr{E}_{1}^{i}\right)(K) \geq \delta_{0}^{2} \sqrt{E^{*}+1}\left(K-K_{s}\right)=\delta_{0}^{2} \sqrt{E^{*}+1}\left|K+\left[c_{1}^{i}\right]\right|
$$

Remark. We are done with Case $2\left(s_{0} \leq 6 l_{0}^{2}\right)$. We will list the most important properties of $S_{1}$ in our induction hypothesis.

Induction hypothesis. For $n \geq 1$ we define

$$
S_{n}\left(K^{*}, E^{*}\right) \equiv\left\{c_{n}^{i} \in \bar{S}_{n-1}: \operatorname{dist}\left(\sigma\left(H\left(I_{n}^{i}, K^{*}\right)\right), E^{*}\right) \leq \delta_{n} \sqrt{E^{*}+1}\right\} .
$$

We assume that every point $c_{n}^{i} \in S_{n}$ belongs to either class A or class B. We also assume that the Decay Theorem holds for $S_{n}$. (See Appendix C.)

Class A. For every $K$ in the interval $\left|K-K^{*}\right| \leq 2 \delta_{n-1}^{3 / 2}$, there exists a unique eigenvalue $E_{n}^{i}(K) \in \sigma\left(H\left(I_{n}^{i}\right)\right)$ such that

(H1) $\left|E_{n}^{i}(K)-E^{*}\right| \leq \operatorname{cst} \delta_{n-1}^{3 / 2} \sqrt{E^{*}+1}$.

(H2) $\left|d E_{n}^{i} / d K\right| \leq\left(3+\mu_{n}\right) \sqrt{E^{*}+1}$ where $\mu_{0}=0$ and $\left|\mu_{n}-\mu_{n-1}\right| \leq 1 / 4^{n}$. 
(H3) If $\left|d E_{n}^{i} / d K\right| \leq \delta_{n-1}^{2} \sqrt{E^{*}+1}$ for some $K$ in the interval $\left|K-K^{*}\right| \leq$ $\delta_{n-1}^{3 / 2}$ then

$$
\left|\frac{d}{d K} E_{n}^{i}\right| \geq \frac{1}{2} \sqrt{E^{*}+1}\left|K+\left[c_{n}^{i}\right]\right|
$$

and

$$
\left|\frac{d^{2}}{d K^{2}} E_{n}^{i}\right| \geq\left(1-\nu_{n}\right) \sqrt{E^{*}+1}
$$

where $\nu_{0}=0$ and $\left|\nu_{n}-\nu_{n-1}\right| \leq 1 / 4^{n}$; moreover $d^{2} E_{n}^{i} / d K^{2}$ has a unique sign.

(H4) $\left|\psi_{n}^{i}(x)\right| \leq \operatorname{cst} e^{-\left(\gamma_{0} / 4\right) l_{n}}$ for $x \in \partial I_{n}^{i}$.

(H5) $\left|E_{n}^{i}\left(K^{*}\right)\right|-E_{n}^{j}\left(K^{*}\right) \mid \geq \frac{1}{2} \delta_{n-1}^{2} \sqrt{E^{*}+1} m\left(c_{n}^{i}, c_{n}^{j}\right)^{2}$.

Class $B$. For every $K$ in the interval $\left|K-K^{*}\right| \leq 6 \delta_{n-1}^{1 / 2} / \delta_{n-2}$, there exist exactly two eigenvalues $E_{n}^{i}(K), \mathscr{E}_{n}^{i}(K) \in \sigma\left(H\left(I_{n}^{i}\right)\right)$ such that

(H6) $\left|E_{n}^{i}(K)-E^{*}\right| \leq \operatorname{cst} \delta_{n-1}^{1 / 2} / \delta_{n-2}$. The same holds for $\mathscr{E}_{n}^{i}$.

(H7) $\left|K^{*}+\left[c_{n}^{i}\right]\right| \leq 3 \delta_{n-1}^{1 / 2} / \delta_{n-2}$.

Note: $\delta_{-1} \equiv 1$.

(H8) $\left|E_{n}^{i}(K)-\mathscr{E}_{n}^{i}(K)\right| \geq \delta_{n-1}^{2} \sqrt{E^{*}+1}\left|K+\left[c_{n}^{i}\right]\right|$

(H9) $\left|d E_{n}^{i} / d K\right| \leq\left(3+\mu_{n}\right) \sqrt{E^{*}+1}$ where $\mu_{0}=0$ and $\left|\mu_{n}-\mu_{n-1}\right| \leq 1 / 4^{n}$. The same holds for $\mathscr{E}_{n}^{i}$.

(H10) If $\left|d E_{n}^{i} / d K\right| \leq \delta_{n-1}^{2} \sqrt{E^{*}+1}$ for some $K$ in the interval $\left|K-K^{*}\right| \leq$ $6 \delta_{n-1}^{1 / 2} / \delta_{n-2}$ then

$$
\left|\frac{d}{d K} E_{n}^{i}\right| \geq \frac{1}{2} \sqrt{E^{*}+1}\left|K+\left[c_{n}^{i}\right]\right|
$$

and

$$
\left|\frac{d^{2}}{d K^{2}} E_{n}^{i}\right| \geq\left(1-\nu_{n}\right) \sqrt{E^{*}+1}
$$

where $\nu_{0}=0$ and $\left|\nu_{n}-\nu_{n-1}\right| \leq 1 / 4^{n}$; moreover $d^{2} E_{n}^{i} / d K^{2}$ has a unique sign. The same holds for $\mathscr{E}_{n}^{i}$.

(H11) $\left|\psi_{n}^{i}(x)\right| \leq \operatorname{cst} e^{-\left(\gamma_{0} / 4\right) l_{n}}$ for $x \in \partial I_{n}^{i}$. The same holds for $\Psi_{n}^{i}$.

(H12) $\left|E_{n}^{i}\left(K^{*}\right)-E_{n}^{j}\left(K^{*}\right)\right| \geq \frac{1}{2} \delta_{n-1}^{2} \sqrt{E^{*}+1} m\left(c_{n}^{i}, c_{n}^{j}\right)^{2}$. The same holds for $\left|\mathscr{E}_{n}^{i}\left(K^{*}\right)-\mathscr{E}_{n}^{j}\left(K^{*}\right)\right|$ and $\left|E_{n}^{i}\left(K^{*}\right)-\mathscr{E}_{n}^{j}\left(K^{*}\right)\right|$.

\section{DEFINITION AND PROPERTIES OF $S_{n+1}$}

In this section, we will assume that the induction hypothesis is true for $S_{n}$ and then prove that it holds for $S_{n+1}$. Many of the proofs run along the same lines as their counterparts in $\S 2$ and will be omitted.

We construct $\bar{S}_{n}$ from $S_{n}$ in the usual way by pairing elements together if $s_{n} \leq 6 l_{n}^{2}$. Then we define

$$
S_{n+1}\left(K^{*}, E^{*}\right) \equiv\left\{c_{n+1}^{i} \in \bar{S}_{n}: \operatorname{dist}\left(\sigma\left(H\left(I_{n+1}^{i}\right)\right), E^{*}\right) \leq \delta_{n+1} \sqrt{E^{*}+1}\right\} .
$$


Case 1. $s_{n} \geq 6 l_{n}^{2}$.

This case will be broken up into two subcases, according to the number of eigenvalues in $\sigma\left(H\left(I_{n}^{i}\right)\right)$ that are near $E^{*}$. Before we consider the two subcases, we will prove some general facts.

Lemma 3.1. If $c_{n+1}^{r} \in S_{n+1}$ for $r=i, j$ then

$$
m\left(c_{n+1}^{i}, c_{n+1}^{j}\right) \leq \delta_{n}^{3 / 2}
$$

Proof. Since $c_{n+1}^{r}=c_{n}^{r}$, by (H5), (H12) it suffices to show that

$$
\left|E_{n}^{i}\left(K^{*}\right)-E_{n}^{j}\left(K^{*}\right)\right|=O\left(\delta_{n}^{4} \sqrt{E^{*}+1}\right)
$$

where $E_{n}^{r} \in \sigma\left(H\left(I_{n}^{r}\right)\right)$.

By definition of $S_{n+1}$, we have eigenvalues $E_{n+1}^{r}\left(K^{*}\right) \in \sigma\left(H\left(I_{n+1}^{r}\right)\right)$ such that $\left|E_{n+1}^{r}\left(K^{*}\right)-E^{*}\right| \leq \delta_{n+1} \sqrt{E^{*}+1}$. We define $A=I_{n+1}^{r} \backslash \widehat{I}_{n}^{r}$ where $\widehat{I}_{n}^{r}$ is a box with center $c_{n+1}^{r}$ whose length is chosen to be $O\left(l_{n}^{1 / 2}\right)$ so that $S_{n} \cap A=\varnothing$. By induction we have

$$
\left|\left(H(A)-E_{n+1}^{r}\right)^{-1}(x, y)\right| \leq \frac{\mathrm{cst}}{\delta_{0} \sqrt{E^{*}+1}} e^{-\left(\gamma_{0} / 2\right)|x-y|}
$$

provided $|x-y| \geq l_{n}^{5 / 6}$. This will allow us to prove that the wave function $\psi_{n+1}^{r}$ decays outside $I_{n}^{r}$. To see this we restrict the eigenvalue equation

$$
H\left(I_{n+1}^{r}\right) \psi_{n+1}^{r}=E_{n+1}^{r} \psi_{n+1}^{r}
$$

to the set $A$,

$$
\psi_{n+1}^{r}=\left(H(A)-E_{n+1}^{r}\right)^{-1} \Gamma\left(\widehat{I}_{n}^{r}\right) \psi_{n+1}^{r} .
$$

Suppose $x \notin I_{n}^{r}$. Since $\operatorname{dist}\left(x, \partial \widehat{I}_{n}^{r}\right) \geq l_{n}^{5 / 6}$, we have

$$
\left|\psi_{n+1}^{r}(x)\right| \leq \sum_{\partial \hat{I}_{n}^{r}} \frac{\operatorname{cst} \varepsilon}{\delta_{0} \sqrt{E^{*}+1}} e^{-\left(\gamma_{0} / 2\right)|x-y|}
$$

and since $|x-y| \geq\left|x-c_{n+1}^{r}\right|-l_{n}^{1 / 2}$, we get

$$
\left|\psi_{n+1}^{r}(x)\right| \leq \operatorname{cst} e^{-\left(\gamma_{0} / 4\right)\left|x-c_{n+1}^{r}\right|} \text { for } x \notin I_{n}^{r} .
$$

We will use $\psi_{n+1}^{r}$ as a trial wave function for $H\left(I_{n}^{r}\right)$. To do this, we restrict (3.1) to $I_{n}^{r}$, yielding

$$
\left(H\left(I_{n}^{r}\right)-E_{n+1}^{r}\right) \psi_{n+1}^{r}=\Gamma\left(I_{n}^{r}\right) \psi_{n+1}^{r}
$$

therefore by (3.2) we get

$$
\left\|\left(H\left(I_{n}^{r}\right)-E_{n+1}^{r}\right) \psi_{n+1}^{r}\right\| \leq \delta_{n}^{4} \sqrt{E^{*}+1} .
$$

Thus by trial wave function we have

$$
\left|E_{n}^{r}\left(K^{*}\right)-E_{n+1}^{r}\left(K^{*}\right)\right| \leq \delta_{n}^{4} \sqrt{E^{*}+1}
$$


therefore

$$
\left|E_{n}^{r}\left(K^{*}\right)-E^{*}\right| \leq 2 \delta_{n}^{4} \sqrt{E^{*}+1} \text { for } r=i, j .
$$

Remark 1. This lemma will be strengthened later by replacing $\delta_{n}^{3 / 2}$ with $2 \delta_{n+1}^{1 / 2} / \delta_{n}$.

Remark 2. By (H2), (H9), and (3.3) we have

$$
\left|E_{n}^{i}(K)-E^{*}\right| \leq \operatorname{cst} \delta_{n}^{3 / 2} \sqrt{E^{*}+1} \text { for }\left|K-K^{*}\right| \leq 4 \delta_{n}^{3 / 2} .
$$

We are now ready to break up case 1 into two subcases.

Subcase 1A.

$$
\operatorname{dist}\left(\sigma\left(H\left(I_{n}^{i}\right)\right)-E_{n}^{i}, E^{*}\right) \geq \delta_{n-1} \sqrt{\delta_{n}} \sqrt{E^{*}+1}
$$

for every $K$ in the interval $\left|K-K^{*}\right| \leq 4 \delta_{n}^{3 / 2}$. In this case we will show how to get back to class $\mathrm{A}$ of the induction hypothesis.

Lemma 3.2. If $c_{n+1}^{i} \in S_{n+1}$ then

(a) There exists a unique eigenvalue $E_{n+1}^{i}(K) \in \sigma\left(H\left(I_{n+1}^{i}\right)\right)$ such that

$$
\left|E_{n+1}^{i}(K)-E^{*}\right| \leq \operatorname{cst} \delta_{n}^{3 / 2} \sqrt{E^{*}+1}
$$

for every $K$ in the interval $\left|K-K^{*}\right| \leq 4 \delta_{n}^{3 / 2}$.

(b) $\left|\psi_{n+1}^{i}(x)\right| \leq \operatorname{cst} e^{-\left(\gamma_{0} / 4\right)\left|x-c_{n+1}^{i}\right|}$ for $x \notin I_{n}^{i}$.

(c) $\left\|\psi_{n+1}^{i}-\psi_{n}^{i}\right\| \leq \operatorname{cst} \delta_{n}^{3}$.

(d) Any other eigenvalue $\mathscr{E}_{n+1}^{i} \in \sigma\left(H\left(I_{n+1}^{i}\right)\right)$ must obey

$$
\left|\mathscr{E}_{n+1}^{i}-E^{*}\right| \geq\left(\delta_{n} / 5\right) \sqrt{E^{*}+1} .
$$

(e) $E_{n+1}^{j}\left(K^{*}\right)=E_{n+1}^{i}\left(K^{*}+\Delta K\right)$ where $\Delta K=\left[c_{n+1}^{j}\right]-\left[c_{n+1}^{i}\right]$ or $-\left(\left[c_{n+1}^{j}\right]+\right.$ $\left.\left[c_{n+1}^{i}\right]+2 K^{*}\right)$ and $|\Delta K|=m\left(c_{n+1}^{i}, c_{n+1}^{j}\right)$.

Proof. We use (H4), (H11) to get

$$
\left\|\left(H\left(I_{n+1}^{i}\right)-E_{n}^{i}\right) \psi_{n}^{i}\right\|=\left\|\Gamma\left(I_{n}^{i}\right) \psi_{n}^{i}\right\| \leq \delta_{n}^{4} \sqrt{E^{*}+1} .
$$

Hence $\psi_{n}^{i}$ serves as a good trial wave function and we conclude that there exists an eigenvalue $E_{n+1}^{i}(K) \in \sigma\left(H\left(I_{n+1}^{i}\right)\right)$ such that

$$
\left|E_{n+1}^{i}(K)-E_{n}^{i}(K)\right| \leq \delta_{n}^{4} \sqrt{E^{*}+1} .
$$

Therefore by (3.4) we have

$$
\left|E_{n+1}^{i}(K)-E^{*}\right| \leq \operatorname{cst} \delta_{n}^{3 / 2} \sqrt{E^{*}+1} .
$$

We now examine the wave function $\psi_{n+1}^{i}$. Part (b) follows from the fact that $S_{n} \cap\left(I_{n+1}^{i} \backslash \widehat{I}_{n}^{i}\right)=\varnothing$. (See (3.2).) To prove part (c) we must show that $\psi_{n+1}^{i}$ 
is close to $\psi_{n}^{i}$ inside $I_{n}^{i}$. To see this, we restrict (3.1) to $I_{n}^{i}$ and arrange the terms as follows:

$$
\left(H\left(I_{n}^{i}\right)-E_{n+1}^{i}\right) \psi_{n+1}^{i}=\Gamma\left(I_{n}^{i}\right) \psi_{n+1}^{i} .
$$

By Lemma 3.2(b) we can bound the right-hand side so that

$$
\psi_{n+1}^{i}=A \psi_{n}^{i}+\left(H\left(I_{n}^{i}\right)-E_{n+1}^{i}\right)_{\perp}^{-1}\left(O\left(\delta_{n}^{4} \sqrt{E^{*}+1}\right)\right) .
$$

Using (3.5) we get $\left\|\psi_{n+1}^{i}-A \psi_{n}^{i}\right\| \leq \operatorname{cst} \delta_{n}^{3}$, and since $\psi_{n+1}^{i}$ is normalized, we must have $|A-1|=O\left(\delta_{n}^{3}\right)$.

To prove part (d) we assume that there is another eigenvalue $\mathscr{E}_{n+1}^{i} \in$ $\sigma\left(H\left(I_{n+1}^{i}\right)\right)$ such that $\left|\mathscr{E}_{n+1}^{i}-E^{*}\right| \leq\left(\delta_{n} / 5\right) \sqrt{E^{*}+1}$. The same argument shows that its wave function decays outside $I_{n}^{i}$ and is close to $\psi_{n}^{i}$ inside $I_{n}^{i}$. This violates orthogonality.

The next lemma tells us that the derivatives of $E_{n+1}^{i}$ closely approximate those of $E_{n}^{i}$. The proof uses Cauchy's theorem, so we must state the lemma in its complex form.

Lemma 3.3. If $c_{n+1}^{i} \in S_{n+1}$ then

(a) $\left|d E_{n+1}^{i} / d K-d E_{n}^{i} / d K\right| \leq \operatorname{cst} \delta_{n}^{2} \sqrt{E^{*}+1}$ for every $K$ in the complex disk $\left|K-K^{*}\right| \leq 4 \delta_{n}^{3 / 2}$.

(b) $\left|d^{2} E_{n+1}^{i} / d K^{2}-d^{2} E_{n}^{i} / d K^{2}\right| \leq \operatorname{cst} \delta_{n}^{1 / 2} \sqrt{E^{*}+1}$ for every $K$ in the complex disk $\left|K-K^{*}\right| \leq 2 \delta_{n}^{3 / 2}$.

Proof. Since $H(K)=\varepsilon \Delta+V(K)$ is analytic, we can extend the eigenvalues and eigenfunctions to be complex analytic functions of $K$ for $K$ in the disk $\left|K-K^{*}\right| \leq 4 \delta_{n}^{3 / 2}$. For complex $K$ we have $d E_{n+1}^{i} / d K=\left\langle\psi_{n+1}^{i}, V^{\prime} \psi_{n+1}^{i}\right\rangle_{\mathbf{R}}$ and $d E_{n}^{i} / d K=\left\langle\psi_{n}^{i}, V^{\prime} \psi_{n}^{i}\right\rangle_{\mathbf{R}}$ where $\langle\phi, \psi\rangle_{\mathbf{R}}=\sum \phi(i) \psi(i)$. To prove part (a) it suffices to show that

$$
\left\|\psi_{n+1}^{i}-\psi_{n}^{i}\right\| \leq \operatorname{cst} \delta_{n}^{3} \text { and }\left\|V^{\prime}\right\| \leq \delta_{n}^{-1} \sqrt{E^{*}+1}
$$

We note that

$$
\begin{aligned}
\left\|V^{\prime}\right\| \leq & \max _{I_{n+1}}\left|v_{x}^{\prime}\right|=\max 2|[x]+K| \leq \max 2\left|[x]-\left[c_{n+1}^{i}\right]\right| \\
& +\max 2\left|\left[c_{n+1}^{i}\right]+K^{*}\right|+\max 2\left|K-K^{*}\right| \leq \operatorname{cst} l_{n+1} \sqrt{E^{*}+1} \\
\leq & \delta_{n}^{-1} \sqrt{E^{*}+1} .
\end{aligned}
$$

To bound the difference of the eigenfunctions we need to establish decay estimates on the Green's function for complex $K$. We begin by taking a contour $C:\left|z-E^{*}\right|=\frac{1}{2} \delta_{n-1} \sqrt{\delta_{n}} \sqrt{E^{*}+1}$. By (3.4) and (3.5) we see that $E_{n}^{i}\left(K^{*}\right) \in$ $\sigma\left(H\left(I_{n}^{i}\right)\right)$ is an isolated eigenvalue inside $C$. When $K=K^{*}$ the operator $H$ is selfadjoint; therefore

$$
\left\|G_{n}\left(K^{*}, z\right)\right\|=\left\|\left(H\left(I_{n}^{i}, K^{*}\right)-z\right)^{-1}\right\| \leq \operatorname{cst}\left(\delta_{n-1} \sqrt{\delta_{n}} \sqrt{E^{*}+1}\right)^{-1}
$$


for $z \in C$. For $K$ in the complex disk we use the resolvent identity

$$
G_{n}(K, z)=G_{n}\left(K^{*}, z\right)+G_{n}\left(K^{*}, z\right)\left(V\left(K^{*}\right)-V(K)\right) G_{n}(K, z)
$$

to bound

$$
\left\|G_{n}(K, z)\right\| \leq \operatorname{cst}\left(\delta_{n-1} \sqrt{\delta_{n}} \sqrt{E^{*}+1}\right)^{-1}
$$

for $z \in C$ and $\left|K-K^{*}\right| \leq 4 \delta_{n}^{3 / 2}$. Since

$$
G_{n}^{\perp}(K, E)=\frac{1}{2 \pi i} \oint_{C} \frac{G_{n}(K, z)}{z-E} d z
$$

we have

$$
\left\|G_{n}^{\perp}(K, E)\right\| \leq \operatorname{cst}\left(\delta_{n-1} \sqrt{\delta_{n}} \sqrt{E^{*}+1}\right)^{-1}
$$

provided $\left|E-E^{*}\right| \leq \operatorname{cst} \delta_{n} \sqrt{E^{*}+1}$.

If we restrict the eigenvalue equation $H\left(I_{n+1}^{i}\right) \psi_{n+1}^{i}=E_{n+1}^{i} \psi_{n+1}^{i}$ to $I_{n}^{i}$ we get

$$
\psi_{n+1}^{i}=A \psi_{n}^{i}+G_{n}^{\perp}\left(K, E_{n+1}^{i}\right) \Gamma\left(I_{n}^{i}\right) \psi_{n+1}^{i} .
$$

Since (3.2) holds for complex $K$ we have

$$
\left\|\psi_{n+1}^{i}-\psi_{n}^{i}\right\| \leq \operatorname{cst} \delta_{n}^{3}
$$

provided $\left|E_{n+1}^{i}-E^{*}\right| \leq \operatorname{cst} \delta_{n} \sqrt{E^{*}+1}$ for $\left|K-K^{*}\right| \leq 4 \delta_{n}^{3 / 2}$. To show that $E_{n+1}^{i}$ remains close to $E^{*}$ we use two contours

$$
\begin{aligned}
& C_{1}:\left|z-E^{*}\right|=\left(\delta_{n} / 10\right) \sqrt{E^{*}+1}, \\
& C_{2}:\left|z-E^{*}\right|=\left(\delta_{n} / 8\right) \sqrt{E^{*}+1} .
\end{aligned}
$$

By Lemma 3.2(d) we see that $E_{n+1}^{i}\left(K^{*}\right) \in \sigma\left(H\left(I_{n+1}^{i}\right)\right)$ is an isolated eigenvalue inside $C_{2}$. Using the same argument as before, we can bound

$$
\left\|G_{n+1}(K, z)\right\| \leq \operatorname{cst}\left(\delta_{n} \sqrt{E^{*}+1}\right)^{-1}
$$

for $z \in C_{2},\left|K-K^{*}\right| \leq 4 \delta_{n}^{3 / 2}$ and

$$
\left\|G_{n+1}^{\perp}(K, E)\right\| \leq \operatorname{cst}\left(\delta_{n} \sqrt{E^{*}+1}\right)^{-1}
$$

for $\left|E-E^{*}\right| \leq\left(\delta_{n} / 10\right) \sqrt{E^{*}+1}$. But

$$
G_{n+1}^{\perp}\left(K, E_{n+1}^{i}\right)=\frac{1}{2 \pi i} \oint_{C_{1}} \frac{G_{n+1}(K, Z)}{z-E_{n+1}} d z
$$

is uniformly bounded when $E_{n+1}^{i}$ is inside $C_{1}$; therefore by continuity $E_{n+1}^{i}$ must stay inside $C_{1}$ for every $K$ in the disk $\left|K-K^{*}\right| \leq 4 \delta_{n}^{3 / 2}$.

To prove part (b) we apply Cauchy's theorem to part (a).

Lemma 3.4. If $c_{n+1}^{i} \in S_{n+1}$ then

(a) $\left|d E_{n+1}^{i} / d K\right| \leq\left(3+\mu_{n+1}\right) \sqrt{E^{*}+1}$. 
(b) If $\left|d E_{n+1}^{i} / d K\right| \leq \delta_{n}^{2} \sqrt{E^{*}+1}$ for some $K$ in the interval $\left|K-K^{*}\right| \leq 2 \delta_{n}^{3 / 2}$ then

$$
\left|\frac{d^{2}}{d K^{2}} E_{n+1}^{i}\right| \geq\left(1-\nu_{n+1}\right) \sqrt{E^{*}+1} ;
$$

moreover $d^{2} E_{n+1}^{i} / d K^{2}$ has a unique sign.

Proof. We use Lemma 3.3(a) and (H2), (H9) to establish part (a). We now prove part (b). If the hypothesis holds, then by Lemma 3.3(a),

$$
\left|\frac{d}{d K} E_{n}^{i}\right| \leq \operatorname{cst} \delta_{n}^{2} \sqrt{E^{*}+1} .
$$

By Lemma 3.3(b) and (H3), (H10) we get

$$
\left|\frac{d^{2}}{d K^{2}} E_{n+1}^{i}\right| \geq\left(1-\nu_{n}\right) \sqrt{E^{*}+1}-O\left(\delta_{n}^{1 / 2} \sqrt{E^{*}+1}\right) \equiv\left(1-\nu_{n+1}\right) \sqrt{E^{*}+1} .
$$

Lemma 3.5. If $\left|d E_{n+1}^{i} / d K\right| \leq \delta_{n}^{2} \sqrt{E^{*}+1}$ for some $K$ in the interval $\left|K-K^{*}\right| \leq$ $\delta_{n}^{3 / 2}$, then

$$
\left|\frac{d}{d K} E_{n+1}^{i}\right| \geq \frac{1}{2} \sqrt{E^{*}+1}\left|K+\left[c_{n+1}^{i}\right]\right| .
$$

Proof. If the hypothesis holds, then by Lemma $3.3(\mathrm{a})$ we have $\left|d E_{n}^{i} / d K\right| \leq$ $\operatorname{cst} \delta_{n}^{2} \sqrt{E^{*}+1}$. Therefore by (H3), (H10) it follows that $\left|K+\left[c_{n+1}^{i}\right]\right|=O\left(\delta_{n}^{2}\right)$. This implies that the symmetry point $K_{s}=-\left[c_{n+1}^{i}\right]$ belongs to the interval $\left|K-K^{*}\right| \leq 2 \delta_{n}^{3 / 2}$ which is contained in the interval of definition of $E_{n+1}^{i}(K)$. We can now use Lemma 3.4(b) and Appendix A to complete the proof.

Lemma 3.6. If $c_{n+1}^{r} \in S_{n+1}$ for $r=i, j$ then

(a) $2 \delta_{n+1} \sqrt{E^{*}+1} \geq\left|E_{n+1}^{i}\left(K^{*}\right)-E_{n+1}^{j}\left(K^{*}\right)\right| \geq \frac{1}{2} \delta_{n}^{2} \sqrt{E^{*}+1} m\left(c_{n+1}^{i}, c_{n+1}^{j}\right)^{2}$.

(b) (Center Theorem) $m\left(c_{n+1}^{i}, c_{n+1}^{j}\right) \leq 2 \delta_{n+1}^{1 / 2} / \delta_{n}$.

Proof. The proof goes word for word the same as the proof of Lemma 2.6 if we replace the indices 0,1 with $n, n+1$.

Remark. We are now back to class $\mathrm{A}$ of the induction hypothesis.

Subcase 1B.

$$
\operatorname{dist}\left(\sigma\left(H\left(I_{n}^{i}\right)\right)-E_{n}^{i}, E^{*}\right) \leq \delta_{n-1} \sqrt{\delta_{n}} \sqrt{E^{*}+1}
$$

for some $\widehat{K}$ in the interval $\left|K-K^{*}\right| \leq 4 \delta_{n}^{3 / 2}$. In this case we will show how to get back to class B of the induction hypothesis.

Let $m \leq n-1$ be the first scale back where $s_{m} \leq 6 l_{m}^{2}$. Thus we have two boxes $I_{m}^{i}, \widetilde{I}_{m}^{i}$ such that

$$
I_{m}^{i}, \widetilde{I}_{m}^{i} \subset I_{m+1}^{i} \subset I_{m+2}^{i} \subset \cdots \subset I_{n}^{i} \subset I_{n+1}^{i}
$$


Note that

$$
c_{n+1}^{i}=c_{m+1}^{i}=\frac{1}{2}\left(c_{m}^{i}+\tilde{c}_{m}^{i}\right)
$$

and by $(0.8)$ we have

$$
\left|\left[c_{m}^{i}\right]+\left[\tilde{c}_{m}^{i}\right]+2 K^{*}\right| \leq 6 \delta_{m}^{1 / 2} / \delta_{m-1} .
$$

The next lemma tells us how wide an interval we need to take in order to include the symmetry point $K_{s}=-\left[c_{n+1}^{i}\right]$.

Lemma 3.7. If $c_{n+1}^{r} \in S_{n+1}$ for $r=i, j$ then

(a) $\left|K^{*}+\left[c_{n+1}^{i}\right]\right| \leq 3 \delta_{n}^{1 / 2} / \delta_{n-1}$.

(b) $\max \left(\left|\left[c_{n+1}^{i}\right]-\left[c_{n+1}^{j}\right]\right|,\left|\left[c_{n+1}^{i}\right]+\left[c_{n+1}^{j}\right]+2 K^{*}\right|\right) \leq 6 \delta_{n}^{1 / 2} / \delta_{n-1}$.

Proof. By (3.4) we have $\left|E_{n}^{i}(K)-E^{*}\right| \leq \operatorname{cst} \delta_{n}^{3 / 2} \sqrt{E^{*}+1}$ for every $K$ in the interval $\left|K-K^{*}\right| \leq 4 \delta_{n}^{3 / 2}$. Equation (3.8) gives us another eigenvalue $\mathscr{E}_{n}^{i}(\widehat{K}) \in$ $\sigma\left(H\left(I_{n}^{i}\right)\right)$ such that

$$
\left|\mathscr{E}_{n}^{i}(\widehat{K})-E^{*}\right| \leq \delta_{n-1} \sqrt{\delta_{n}} \sqrt{E^{*}+1}
$$

Thus we have two eigenvalues in $\sigma\left(H\left(I_{n}^{i}\right)\right)$ close enough to $E^{*}$ so that our boxes $I_{n}^{i}$ belong to class B of the induction hypothesis. By (3.11), (3.8), and (H9) we have $\left|\mathscr{E}_{n}^{i}\left(K^{*}\right)-E^{*}\right| \leq 2 \delta_{n-1} \sqrt{\delta_{n}} \sqrt{E^{*}+1}$; therefore

$$
\left|\mathscr{E}_{n}^{i}\left(K^{*}\right)-E_{n}^{i}\left(K^{*}\right)\right| \leq 3 \delta_{n-1} \sqrt{\delta_{n}} \sqrt{E^{*}+1}
$$

Since $c_{n+1}^{i}=c_{n}^{i}$, we can use (H8) to establish part (a) of the lemma. Part (b) follows immediately from part (a).

Lemma 3.8. If $c_{n+1}^{r} \in S_{n+1}$ for $r=i, j$ then

(a) There exist two eigenvalues $E_{n+1}^{i}(K)$ and $\mathscr{E}_{n+1}^{i}(K) \in \sigma\left(H\left(I_{n+1}^{i}\right)\right)$ such that

$$
\left|E_{n+1}^{i}(K)-E^{*}\right| \leq \operatorname{cst}\left(\delta_{n}^{1 / 2} / \delta_{n-1}\right) \sqrt{E^{*}+1}
$$

for every $K$ in the interval $\left|K-K^{*}\right| \leq 6 \delta_{n}^{1 / 2} / \delta_{n-1}$. The same holds for $\mathscr{E}_{n+1}^{i}(K)$.

(b) $\left|\psi_{n+1}^{i}(x)\right| \leq \operatorname{cst}\left(e^{\left.-\gamma_{0} / 4\right)\left|x-c_{m}^{i}\right|}+e^{-\left(\gamma_{0} / 4\right)\left|x-\hat{c}_{m}^{i}\right|}\right)$ for $x \notin I_{m}^{i} \cup \widetilde{I}_{m}^{i}$. The same holds for $\Psi_{n+1}^{i}$.

(c) $E_{n+1}^{j}\left(K^{*}\right)=E_{n+1}^{i}\left(K^{*}+\Delta K\right)$ or $E_{n+1}^{j}\left(K^{*}\right)=\mathscr{E}_{n+1}^{i}\left(K^{*}+\Delta K\right)$ where $\Delta K=$ $\left[c_{n+1}^{j}\right]-\left[c_{n+1}^{i}\right]$ and $|\Delta K| \leq 6 \delta_{n}^{1 / 2} / \delta_{n-1}$.

Proof. From (3.4) and (H9) it follows that

$$
\left|E_{n}^{i}(K)-E^{*}\right| \leq \operatorname{cst}\left(\delta_{n}^{1 / 2} / \delta_{n-1}\right) \sqrt{E^{*}+1}
$$

for every $K$ in the interval $\left|K-K^{*}\right| \leq 6 \delta_{n}^{1 / 2} / \delta_{n-1}$. By (H11) we have

$$
\left\|\left(H\left(I_{n+1}^{i}\right)-E_{n}^{i}\right) \psi_{n}^{i}\right\|=\left\|\Gamma\left(I_{n}^{i}\right) \psi_{n}^{i}\right\| \leq \operatorname{cst} \delta_{n}^{4} \sqrt{E^{*}+1} ;
$$


therefore (3.12) implies

$$
\left\|\left(H\left(I_{n+1}^{i}\right)-E^{*}\right) \psi_{n}^{i}\right\| \leq \operatorname{cst}\left(\delta_{n}^{1 / 2} / \delta_{n-1}\right) \sqrt{E^{*}+1} .
$$

The same equation holds if we replace $\psi_{n}^{i}$ with $\Psi_{n}^{i}$. Thus by trial wave function we establish part $(a)$.

We now prove part (b). Fix $x \notin I_{m}^{i} \cup \widetilde{I}_{m}^{i}$. Suppose $x \in I_{n}^{i} \backslash I_{m+1}^{i}$. Then $x \in$ $I_{r+1}^{i} \backslash I_{r}^{i}$ for some $m+1 \leq r \leq n-1$. We choose an annulus $A$ around $I_{r+1}^{i} \backslash I_{r}^{i}$ such that $\operatorname{dist}(x, \partial A) \geq l_{r}^{5 / 6}$. By Lemma 3.7(a) and the Center Theorem for $S_{r}$ we see that $S_{r} \cap A=\varnothing$. Now we restrict the eigenvalue equation $H\left(I_{n+1}^{i}\right) \psi_{n+1}^{i}=$ $E_{n+1}^{i} \psi_{n+1}^{i}$ to the annulus $A$ and then use the Decay Theorem for $S_{r}$ to prove part (b). The case where $x \notin I_{n}^{i} \backslash I_{m+1}^{i}$ is treated the same way.

To establish part (c) we use (0.10) and Lemma 3.7(b).

Remark. By Appendix B, we can always assume that the eigenvalues and eigenfunctions are labeled so that they are differentiable functions of $K$.

Lemma 3.9. For every $K$ in the interval $\left|K-K^{*}\right| \leq 6 \delta_{n}^{1 / 2} / \delta_{n-1}$ there are eigenvalues $E_{m}^{i}(K) \in \sigma\left(H\left(I_{m}^{i}\right)\right)$ and $\widetilde{E}_{m}^{i}(K) \in \sigma\left(H\left(\widetilde{I}_{m}^{i}\right)\right)$ with the following properties:
(a) $\left|E_{m}^{i}(K)-E_{n+1}^{i}(K)\right| \leq 2 \delta_{m}^{4} \sqrt{E^{*}+1}$.
(b) $\left|\widetilde{E}_{m}^{i}(K)-E_{n+1}^{i}(K)\right| \leq 2 \delta_{m}^{4} \sqrt{E^{*}+1}$.
(c) $\left|E_{m}^{i}(K)-\mathscr{E}_{n+1}^{i}(K)\right| \leq 2 \delta_{m}^{4} \sqrt{E^{*}+1}$.
(d) $\left|\widetilde{E}_{m}^{i}(K)-\mathscr{E}_{n+1}^{i}(K)\right| \leq 2 \delta_{m}^{4} \sqrt{E^{*}+1}$.
(e) $\left\|\left(H\left(I_{m}^{i}\right)-E_{m}^{i}\right)_{\perp}^{-1}\right\| \leq\left(\delta_{m-1}^{3} \sqrt{E^{*}+1}\right)^{-1}$.
(f) $\left\|\left(H\left(\widetilde{I}_{m}^{i}\right)-\widetilde{E}_{m}^{i}\right)_{\perp}^{-1}\right\| \leq\left(\delta_{m-1}^{3} \sqrt{E^{*}+1}\right)^{-1}$.

Proof. One of the wave functions, say $\psi_{n+1}^{i}$, must have significant amplitude in $I_{m}^{i}$. This follows from Lemma 3.8(b) and the fact that $\psi_{n+1}^{i}$ and $\Psi_{n+1}^{i}$ are orthogonal. We use $\psi_{n+1}^{i}$ as a trial wave function for $H\left(I_{m}^{i}\right)$, yielding

$$
\left\|\left(H\left(I_{m}^{i}\right)-E_{n+1}^{i}\right) \psi_{n+1}^{i}\right\|=\left\|\Gamma\left(I_{m}^{i}\right) \psi_{n+1}^{i}\right\|,
$$

which by Lemma $3.8(\mathrm{~b})$ is bounded by $\delta_{m}^{4} \sqrt{E^{*}+1}$. This implies

$$
\left|E_{m}^{i}(K)-E_{n+1}^{i}(K)\right| \leq \delta_{m}^{4} \sqrt{E^{*}+1}
$$

which establishes part (a). Lemma 3.8(a) together with (3.13) proves part (c). We will now prove the uniqueness of $E_{m}^{i}(K)$. By (H8) we have

$$
\left|\mathscr{E}_{m}^{i}(K)-E_{m}^{i}(K)\right| \geq \delta_{m-1}^{2} \sqrt{E^{*}+1}\left|K+\left[c_{m}^{i}\right]\right|
$$

where

$$
\begin{aligned}
\left|K+\left[c_{m}^{i}\right]\right| & =\left|K-K^{*}+K^{*}+\left[c_{n+1}^{i}\right]+\left[c_{m}^{i}\right]-\left[c_{n+1}^{i}\right]\right| \\
& \geq\left|\left[c_{m}^{i}\right]-\left[c_{n+1}^{i}\right]\right|-O\left(\delta_{n}^{1 / 2} / \delta_{n-1}\right) .
\end{aligned}
$$


By (3.9) and (0.2) we have

$$
\left|\left[c_{m}^{i}\right]-\left[c_{n+1}^{i}\right]\right|=\frac{1}{2}\left|\left[c_{m}^{i}\right]-\left[\tilde{c}_{m}^{i}\right]\right| \geq \operatorname{cst} /\left|c_{m}^{i}-\tilde{c}_{m}^{i}\right|^{2} \geq \operatorname{cst} / l_{m}^{4}
$$

therefore

$$
\left|K+\left[c_{m}^{i}\right]\right| \geq \operatorname{cst} l_{m}^{4}-O\left(\delta_{n}^{1 / 2} / \delta_{n-1}\right) \geq \delta_{m-1},
$$

which implies

$$
\left|\mathscr{E}_{m}^{i}(K)-E_{m}^{i}(K)\right| \geq \delta_{m-1}^{3} \sqrt{E^{*}+1} .
$$

This shows that $E_{m}^{i}(K)$ is the unique eigenvalue in $\sigma\left(H\left(I_{m}^{i}\right)\right)$ which is near $E^{*}$, thus proving part (e) of the lemma.

A similar argument in $\widetilde{I}_{m}^{i}$ shows that there exists an eigenvalue $\widetilde{E}_{m}^{i}(K) \in$ $\sigma\left(H\left(\widetilde{I}_{m}^{i}\right)\right)$ such that parts (b), (d), and (f) hold. If we transform the equation $H\left(\widetilde{I}_{m}^{i}\right) \tilde{\psi}_{m}^{i}=\widetilde{E}_{m}^{i} \tilde{\psi}_{m}^{i}$ to the box $I_{m}^{i}$ by changing variables $x \rightarrow-x+c_{m}^{i}+\tilde{c}_{m}^{i}$, we get

$$
\widetilde{E}_{m}^{i}(K)=E_{m}^{i}\left(-K-\left[c_{m}^{i}\right]-\left[\tilde{c}_{m}^{i}\right]\right) .
$$

By induction we know the structure of $E_{m}^{i}(K)$; therefore equation (3.15) gives us a full description of $\widetilde{E}_{m}^{i}(K)$.

Lemma 3.10. If $c_{n+1}^{i} \in S_{n+1}$ then

(a) $\left\|\psi_{n+1}^{i}-A \psi_{m}^{i}\right\| \leq \operatorname{cst} \delta_{m}^{3}$,

(b) $\left\|\psi_{n+1}^{i}-B \tilde{\psi}_{m}^{i}\right\| \leq \operatorname{cst} \delta_{m}^{3}$,

(c) $\left\|\Psi_{n+1}^{i}-B \psi_{m}^{i}\right\| \leq \operatorname{cst} \delta_{m}^{3}$,

(d) $\left\|\Psi_{n+1}^{i}+A \tilde{\psi}_{m}^{i}\right\| \leq \operatorname{cst} \delta_{m}^{3}$,

where $1 \geq A^{2}+B^{2} \geq 1-\delta_{m}$.

Proof. We restrict the equation $H\left(I_{n+1}^{i}\right) \psi_{n+1}^{i}=E_{n+1}^{i} \psi_{n+1}^{i}$ to $I_{m}^{i}$ and write it as

$$
\left(H\left(I_{m}^{i}\right)-E_{m}^{i}\right) \psi_{n+1}^{i}=\left(E_{n+1}^{i}-E_{m}^{i}\right) \psi_{n+1}^{i}+\Gamma\left(I_{m}^{i}\right) \psi_{n+1}^{i} \cdot
$$

By Lemmas 3.9(a) and 3.8(b), the right-hand side is bounded by $\operatorname{cst} \delta_{m}^{4} \sqrt{E^{*}+1}$; therefore

$$
\psi_{n+1}^{i}=A \psi_{m}^{i}+\left(H\left(I_{m}^{i}\right)-E_{m}^{i}\right)_{\perp}^{-1}\left(O\left(\delta_{m}^{4} \sqrt{E^{*}+1}\right)\right) .
$$

By Lemma 3.9(e) we get $\left\|\psi_{n+1}^{i}-A \psi_{m}^{i}\right\| \leq \operatorname{cst} \delta_{m}^{3}$. We can do the same thing in $\widetilde{I}_{m}^{i}$ to get $\left\|\psi_{n+1}^{i}-B \tilde{\psi}_{m}^{i}\right\| \leq \operatorname{cst} \delta_{m}^{3}$. Orthogonality and normalization give us parts (c) and (d) and the relationship between $A$ and $B$.

We have two eigenvalues $E_{n+1}^{i}$ and $\mathscr{E}_{n+1}^{i}$ in $\sigma\left(H\left(I_{n+1}^{i}\right)\right)$ that are close enough to $E^{*}$ to make their wave functions decay exponentially fast outside $I_{m}^{i} \cup \widetilde{I}_{m}^{i}$. Any other eigenvalue must be far enough away from $E^{*}$ to make its wavefunction orthogonal to $\psi_{n+1}^{i}$ and $\Psi_{n+1}^{i}$. 
Lemma 3.11. Any other eigenvalue $\widehat{E} \in \sigma\left(H\left(I_{n+1}^{i}\right)\right)$ obeys

$$
\left|\widehat{E}-E^{*}\right| \geq \delta_{m}^{4} \sqrt{E^{*}+1} \geq \delta_{n-1}^{4} \sqrt{E^{*}+1} .
$$

Proof. Suppose that there is a third eigenvalue $e_{n+1}^{i} \in \sigma\left(H\left(I_{n+1}^{i}\right)\right)$ such that $\left|e_{n+1}^{i}-E^{*}\right| \leq \delta_{m}^{4} \sqrt{E^{*}+1}$. Then we can show that its wave function $\phi_{n+1}^{i}$ decays exponentially fast outside $I_{m}^{i} \cup \widetilde{I}_{m}^{i}$ and also satisfies

$$
\left\|\phi_{n+1}^{i}-A \psi_{m}^{i}\right\| \leq \operatorname{cst} \delta_{m}^{3} \text { and }\left\|\phi_{n+1}^{i}-B \tilde{\psi}_{m}^{i}\right\| \leq \operatorname{cst} \delta_{m}^{3} .
$$

(See Lemmas 3.8(b) and 3.10.) Therefore it is impossible for $\psi_{n+1}^{i}, \Psi_{n+1}^{i}$, and $\phi_{n+1}^{i}$ to be orthogonal.

In Lemma 3.10 we expressed $\psi_{n+1}^{i}$ and $\Psi_{n+1}^{i}$ in terms of $\psi_{m}^{i}$ and $\tilde{\psi}_{m}^{i}$. This will allow us to relate the derivatives of $E_{n+1}^{i}$ and $\mathscr{E}_{n+1}^{i}$ to those of $E_{m}^{i}$ and $\widetilde{E}_{m}^{i}$. To do this we need to prove some technical lemmas about $E_{m}^{i}$ and $\widetilde{E}_{m}^{i}$.

Lemma 3.12. $\left|d E_{m}^{i} / d K+d \widetilde{E}_{m}^{i} / d K\right| \leq \delta_{m}^{1 / 4} \sqrt{E^{*}+1}$.

Proof. By (3.10) and (3.15) we have

$$
\begin{aligned}
& \left|\left(\frac{d}{d K} E_{m}^{i}\right)(K)+\left(\frac{d}{d K} \widetilde{E}_{m}^{i}\right)(K)\right| \\
& \quad=\left|\left(\frac{d}{d K} E_{m}^{i}\right)(K)-\left(\frac{d}{d K} E_{m}^{i}\right)\left(-K-\left[c_{m}^{i}\right]-\left[\tilde{c}_{m}^{i}\right]\right)\right| \\
& \quad \leq \max \left|\frac{d^{2}}{d K^{2}} E_{m}^{i}\right| \times O\left(\left(\delta_{m}^{1 / 2}\right) / \delta_{m-1}\right) .
\end{aligned}
$$

We must estimate

$$
\frac{d^{2}}{d K^{2}} E_{m}^{i}=2+2\left\langle V^{\prime} \psi_{m}^{i},\left(E_{m}^{i}-H\right)_{\perp}^{-1} V^{\prime} \psi_{m}^{i}\right\rangle \leq 2+2\left\|V^{\prime} \psi_{m}^{i}\right\|^{2}\left\|\left(E_{m}^{i}-H\right)_{\perp}^{-1}\right\| .
$$

By Lemma 3.9(e) we have $\left\|\left(E_{m}^{i}-H\right)_{\perp}^{-1}\right\| \leq\left(\delta_{m-1}^{3} \sqrt{E^{*}+1}\right)^{-1}$, so it remains to estimate

$$
\left\|V^{\prime} \psi_{m}^{i}\right\|^{2} \leq\left\|V^{\prime}\left(I_{m}^{i}\right)\right\|^{2} \leq\left(\max _{I_{m}}\left|v_{x}^{\prime}\right|\right)^{2} \leq\left(l_{m} \sqrt{E^{*}+1}\right)^{2} .
$$

Thus

$$
\left|\frac{d}{d K} E_{m}^{i}+\frac{d}{d K} \widetilde{E}_{m}^{i}\right| \leq \operatorname{cst}\left(\frac{l_{m}^{2} \sqrt{E^{*}+1}}{\delta_{m-1}^{3}} \frac{\delta_{m}^{1 / 2}}{\delta_{m-1}}\right) \leq \delta_{m}^{1 / 4} \sqrt{E^{*}+1} .
$$

Lemma 3.13. If $K$ is in the interval $\left|K-K^{*}\right| \leq 6 \delta_{n}^{1 / 2} / \delta_{n-1}$, then $\left|d E_{m}^{i} / d K\right| \geq$ $\delta_{m-1}^{2} \sqrt{E^{*}+1}$.

Proof. If $\left|d E_{m}^{i} / d K\right| \leq \delta_{m-1}^{2} \sqrt{E^{*}+1}$ then by (H3), (H10) we have $\left|d E_{m}^{i} / d K\right|$ $\geq \frac{1}{2} \sqrt{E^{*}+1}\left|K+\left[c_{m}^{i}\right]\right|$, contradicting (3.14).

Now we can prove the lemma which relates the derivatives of $E_{n+1}^{i}, \mathscr{E}_{n+1}^{i}$ to those of $E_{m}^{i}, \widetilde{E}_{m}^{i}$. 
Lemma 3.14. If $c_{n+1}^{i} \in S_{n+1}$ then

(a)

$$
\begin{aligned}
& \frac{d}{d K} E_{n+1}^{i}=\left(A^{2}-B^{2}\right) \frac{d}{d K} E_{m}^{i}+O\left(\delta_{m}^{1 / 4} \sqrt{E^{*}+1}\right), \\
& \frac{d}{d K} \mathscr{E}_{n+1}^{i}=\left(B^{2}-A^{2}\right) \frac{d}{d K} E_{m}^{i}+O\left(\delta_{m}^{1 / 4} \sqrt{E^{*}+1}\right) .
\end{aligned}
$$

(b)

$$
\begin{aligned}
& \frac{d^{2}}{d K^{2}} E_{n+1}^{i}=2 \frac{\left\langle\psi_{n+1}^{i}, V^{\prime} \Psi_{n+1}^{i}\right\rangle^{2}}{E_{n+1}^{i}-\mathscr{E}_{n+1}^{i}}+O\left(\frac{l_{n-1}^{2} \sqrt{E^{*}+1}}{\delta_{n-1}^{4}}\right), \\
& \frac{d^{2}}{d K^{2}} \mathscr{E}_{n+1}^{i}=2 \frac{\left\langle\psi_{n+1}^{i}, V^{\prime} \Psi_{n+1}^{i}\right\rangle^{2}}{\mathscr{E}_{n+1}^{i}-E_{n+1}^{i}}+O\left(\frac{l_{n-1}^{2} \sqrt{E^{*}+1}}{\delta_{n-1}^{4}}\right),
\end{aligned}
$$

(both hold where $\left.E_{n+1}^{i} \neq \mathscr{E}_{n+1}^{i}\right)$.

(c) $\left\langle\psi_{n+1}^{i}, V^{\prime} \Psi_{n+1}^{i}\right\rangle=2 A B d E_{m}^{i} / d K+O\left(\delta_{m}^{1 / 4} \sqrt{E^{*}+1}\right)$.

(d) $\left|d E_{n+1}^{i} / d K+d \mathscr{E}_{n+1}^{i} / d K\right| \leq \operatorname{cst} \delta_{m}^{1 / 4} \sqrt{E^{*}+1}$.

Proof. Since $d E_{n+1}^{i} / d K=\left\langle\psi_{n+1}^{i}, V^{\prime} \psi_{n+1}^{i}\right\rangle, d E_{m}^{i} / d K=\left\langle\psi_{m}^{i}, V^{\prime} \psi_{m}^{i}\right\rangle$, and $d \widetilde{E}_{m}^{i} / d K=\left\langle\tilde{\psi}_{m}^{i}, V^{\prime} \tilde{\psi}_{m}^{i}\right\rangle$, we use Lemmas 3.10 and $3.8(\mathrm{~b})$ and the fact that $\left\|V^{\prime}\left(I_{m}^{i}\right)\right\| \leq \operatorname{cst} l_{m} \sqrt{E^{*}+1}$ to get

$$
\begin{aligned}
\frac{d}{d K} E_{n+1}^{i} & =A^{2} \frac{d}{d K} E_{m}^{i}+B^{2} \frac{d}{d K} \widetilde{E}_{m}^{i}+O\left(\delta_{m}^{2} \sqrt{E^{*}+1}\right) \\
& =\left(A^{2}-B^{2}\right) \frac{d}{d K} E_{m}^{i}+B^{2}\left(\frac{d}{d K} E_{m}^{i}+\frac{d}{d K} \widetilde{E}_{m}^{i}\right)+O\left(\delta_{m}^{2} \sqrt{E^{*}+1}\right) .
\end{aligned}
$$

Now we can use Lemma 3.12 to establish part (a). The same argument is used to prove part $(\mathrm{c})$.

We now prove part (b). By Appendix B we have

$$
\frac{d^{2}}{d K^{2}} E_{n+1}^{i}=2+2 \frac{\left\langle\psi_{n+1}^{i}, V^{\prime} \psi_{n+1}^{i}\right\rangle^{2}}{E_{n+1}^{i}-\mathscr{E}_{n+1}^{i}}+2\left\langle V^{\prime} \psi_{n+1}^{i},\left(E_{n+1}^{i}-H\right)_{\perp \perp}^{-1} V^{\prime} \psi_{n+1}^{i}\right\rangle,
$$

and the remainder term is bounded by

$$
\operatorname{cst}\left\|V^{\prime} \psi_{n+1}^{i}\right\|^{2}\left\|\left(E_{n+1}^{i}-H\right)_{\perp \perp}^{-1}\right\| \text {. }
$$

Lemma 3.11 implies $\left\|\left(E_{n+1}^{i}-H\right)_{\perp \perp}^{-1}\right\| \leq\left(\delta_{n-1}^{4} \sqrt{E^{*}+1}\right)^{-1}$, so it remains to estimate $\left\|V^{\prime} \psi_{n+1}^{i}\right\|^{2}$. By Lemma 3.8(b) we have

$$
\begin{aligned}
\left\|V^{\prime} \psi_{n+1}^{i}\right\|^{2} & \leq\left\|V^{\prime}\left(I_{m}^{i}\right)\right\|^{2}+\left\|V^{\prime}\left(\widetilde{I}_{m}^{i}\right)\right\|^{2}+O\left(\delta_{m}^{2} \sqrt{E^{*}+1}\right) \\
& \leq \operatorname{cst}\left(l_{m} \sqrt{E^{*}+1}\right)^{2}+O\left(\delta_{m}^{2} \sqrt{E^{*}+1}\right) \\
& \leq \operatorname{cst}\left(l_{n-1} \sqrt{E^{*}+1}\right)^{2} \quad(\text { since } m \leq n-1) .
\end{aligned}
$$

Therefore the remainder term is bounded by $\operatorname{cst}\left(l_{n-1}^{2} \sqrt{E^{*}+1} / \delta_{n-1}^{4}\right)$ and part (b) is established. The proof of part (d) follows immediately from part (a). 
Lemma 3.15. If $\left|d E_{n+1}^{i} / d K\right| \leq \delta_{m-1}^{3} \sqrt{E^{*}+1}$ for some $K$ in the interval $\mid K-$ $K^{*} \mid \leq 6 \delta_{n}^{1 / 2} / \delta_{n-1}$ then

(a) $\left|\left\langle\psi_{n+1}^{i}, V^{\prime} \Psi_{n+1}^{i}\right\rangle\right| \geq \frac{1}{3} \delta_{m-1}^{2} \sqrt{E^{*}+1}$.

(b) $\left|d^{2} E_{n+1}^{i} / d K^{2}\right| \geq \sqrt{E^{*}+1}$ at all points where $E_{n+1}^{i} \neq \mathscr{E}_{n+1}^{i}$; moreover $d^{2} E_{n+1}^{i} / d K^{2}$ has a unique sign.

Remark 1. Lemma 3.15 holds if $E_{n+1}^{i}$ is replaced by $\mathscr{E}_{n+1}^{i}$.

Remark 2. We will show later that under the hypothesis of Lemma 3.15, $E_{n+1}^{i} \neq$ $\mathscr{E}_{n+1}^{i}$ for every $K$ in the interval $\left|K-K^{*}\right| \leq 6 \delta_{n}^{1 / 2} / \delta_{n-1}$.

Proof. If $\left|d E_{n+1}^{i} / d K\right| \leq \delta_{m-1}^{3} \sqrt{E^{*}+1}$ for some $K$, then by Lemma 3.14(a) we have $\left|A^{2}-B^{2}\right|\left|d E_{m}^{i} / d K\right| \leq \operatorname{cst} \delta_{m-1}^{3} \sqrt{E^{*}+1}$. By Lemma 3.13 we get $\left|A^{2}-B^{2}\right|=O\left(\delta_{m-1}\right)$, and therefore by Lemma 3.10 we must have $|A B| \geq \frac{1}{4}$. Lemmas $3.14(\mathrm{c})$ and 3.13 give us

$$
\left|\left\langle\psi_{n+1}^{i}, V^{\prime} \Psi_{n+1}^{i}\right\rangle\right| \geq \frac{1}{3} \delta_{m-1}^{2} \sqrt{E^{*}+1} .
$$

To prove part (b) we use Lemmas 3.14(b) and 3.8(a) and part (a).

Lemma 3.16. Let $c_{n+1}^{i} \in S_{n+1}$; then

(a) $\left|d E_{n+1}^{i} / d K\right| \leq\left(3+\mu_{n+1}\right) \sqrt{E^{*}+1}$.

(b) If $\left|d E_{n+1}^{i} / d K\right| \leq \delta_{n}^{2} \sqrt{E^{*}+1}$ for some $K$ in the interval $\left|K-K^{*}\right| \leq$ $6 \delta_{n}^{1 / 2} / \delta_{n-1}$ then

$$
\left|\frac{d}{d K} E_{n+1}^{i}\right| \geq \frac{1}{2} \sqrt{E^{*}+1}\left|K+\left[c_{n+1}^{i}\right]\right| .
$$

Remark. The same holds for $\mathscr{E}_{n+1}^{i}$.

Proof. The proof of part (a) follows immediately from (H9) and Lemma 3.14(a). To establish part (b) we use Appendix A and Lemma 3.15.

Lemma 3.17. Let $c_{n+1}^{i} \in S_{n+1}$; then

$$
\left|E_{n+1}^{i}\left(K_{2}\right)-E_{n+1}^{i}\left(K_{1}\right)\right| \geq \delta_{n}^{2} \sqrt{E^{*}+1} \min \left\{\begin{array}{l}
\left|K_{2}-K_{1}\right|^{2}, \\
\left|K_{2}+K_{1}+2\left[c_{n+1}^{i}\right]\right|^{2}
\end{array}\right.
$$

for any two points $K_{1}, K_{2}$ in the interval $\left|K-K^{*}\right| \leq 6 \delta_{n}^{1 / 2} / \delta_{n-1}$. The same holds for $\mathscr{E}_{n+1}^{i}$.

Proof. The proof goes the same as the proof of Lemma 2.13. There will be two cases to consider:

Case I. $E_{n+1}^{i}\left(K_{s}\right)>\mathscr{E}_{n+1}^{i}\left(K_{s}\right)$ where $K_{s}=-\left[c_{n+1}^{i}\right]$.

In this case, the analog of (2.6) holds; i.e.,

$$
\left(\frac{d}{d K} E_{n+1}^{i}\right)\left(K_{s}\right)=\left(\frac{d}{d K} \mathscr{E}_{n+1}^{i}\right)\left(K_{s}\right)=0 .
$$


By Lemmas 3.15(a) and 3.14(b) we see that $d^{2} E_{n+1}^{i} / d K^{2}$ and $d^{2} \mathscr{E}_{n+1}^{i} / d K^{2}$ are large with opposite signs so that $E_{n+1}^{i}(K)$ and $\mathscr{C}_{n+1}^{i}(K)$ never cross. Appendix $A$ and Lemma 3.15(b) give us

$$
\left|E_{n+1}^{i}\left(K_{2}\right)-E_{n+1}^{i}\left(K_{1}\right)\right| \geq \delta_{n}^{2} \sqrt{E^{*}+1} \min \left\{\begin{array}{l}
\left|K_{2}-K_{1}\right|^{2}, \\
\left|K_{2}+K_{1}+2\left[c_{n+1}^{i}\right]\right|^{2} .
\end{array}\right.
$$

Case II. $E_{n+1}^{i}\left(K_{s}\right)=\mathscr{E}_{n+1}^{i}\left(K_{s}\right)$.

In this case we will show that

$$
\left|\frac{d}{d K} E_{n+1}^{i}\right| \geq \delta_{m-1}^{3} \sqrt{E^{*}+1} \text { and }\left|\frac{d}{d K} \mathscr{E}_{n+1}^{i}\right| \geq \delta_{m-1}^{3} \sqrt{E^{*}+1}
$$

holds for $\left|K-K^{*}\right| \leq 6 \delta_{n}^{1 / 2} / \delta_{n-1}$; moreover, the derivatives have opposite signs. To see this we calculate the derivatives at the symmetry point $K_{s}$ using the special basis $\left\{\psi_{s}, \psi_{a}\right\}$ of symmetric and antisymmetric wave functions (see the proof of Lemma 2.13 for details). This calculation yields

$$
\left(\frac{d}{d K} E_{n+1}^{i}\right)\left(K_{s}\right)=-\left(\frac{d}{d K} \mathscr{E}_{n+1}^{i}\right)\left(K_{s}\right)=\left\langle\psi_{s}, V^{\prime} \psi_{a}\right\rangle .
$$

By symmetry and the decay of the wave function, we may restrict this inner product to $I_{m}^{i}$. Inside $I_{m}^{i}$ we can express $\psi_{s} \cong A \psi_{m}^{i}$ and $\psi_{a} \cong C \psi_{m}^{i}$ where $|A| \cong 1 / \sqrt{2}$ and $|C| \cong 1 / \sqrt{2}$; therefore the analog of $(2.8)$ holds:

$$
\left(\frac{d}{d K} E_{n+1}^{i}\right)\left(K_{s}\right) \cong 2\left\langle A \psi_{m}^{i}, V^{\prime} C \psi_{m}^{i}\right\rangle \cong \frac{d}{d K} E_{m}^{i} .
$$

Now we use Lemma 3.13 to prove (3.17).

Lemma 3.18. Let $c_{n+1}^{r} \in S_{n+1}$ for $r=i, j$; then

(a) $\left|E_{n+1}^{i}\left(K^{*}\right)-E_{n+1}^{j}\left(K^{*}\right)\right| \geq \delta_{n}^{2} \sqrt{E^{*}+1} m\left(c_{n+1}^{i}, c_{n+1}^{j}\right)^{2}$; the same holds for $\left|\mathscr{E}_{n+1}^{i}\left(K^{*}\right)-\mathscr{E}_{n+1}^{j}\left(K^{*}\right)\right|$.

(b) $\left|E_{n+1}^{i}\left(K^{*}\right)-\mathscr{E}_{n+1}^{j}\left(K^{*}\right)\right| \geq \delta_{n}^{2} \sqrt{E^{*}+1} m\left(c_{n+1}^{i}, c_{n+1}^{j}\right)^{2}$.

(c) (Center Theorem) $m\left(c_{n+1}^{i}, c_{n+1}^{j}\right) \leq 2 \delta_{n+1}^{1 / 2} / \delta_{n}$.

Proof. See the proof of Lemma 2.15 and replace the indices 0,1 with $n, n+$ 1.

Lemma 3.19. Let $c_{n+1}^{i} \in S_{n+1}$; then

$$
\left|E_{n+1}^{i}(K)-\mathscr{E}_{n+1}^{i}(K)\right| \geq \delta_{n}^{2} \sqrt{E^{*}+1}\left|K+\left[c_{n+1}^{i}\right]\right|
$$

for every $K$ in the interval $\left|K-K^{*}\right| \leq 6 \delta_{n}^{1 / 2} / \delta_{n-1}$.

Proof. The proof goes like the proof of Lemma 2.16. There are two cases to consider:

Case I. $E_{n+1}^{i}\left(K_{s}\right)=\mathscr{E}_{n+1}^{i}\left(K_{s}\right)$ where $K_{s}=-\left[c_{n+1}^{i}\right]$.

In this case we use (3.17) and follow the proof of Lemma 2.16 (Case I).

Case II. $E_{n+1}^{i}\left(K_{s}\right)>\mathscr{E}_{n+1}^{i}\left(K_{s}\right)$. 
By (3.16) and Lemma 3.15(a) we have

$$
\left|\left\langle\psi_{n+1}^{i}, V^{\prime} \Psi_{n+1}^{i}\right\rangle\left(K_{s}\right)\right| \geq \frac{1}{3} \delta_{m-1}^{2} \sqrt{E^{*}+1} .
$$

Now we follow the proof of Lemma 2.16 (Case II).

Remark. We are back to class B of the induction hypothesis.

Case 2. $s_{n} \leq 6 l_{n}^{2}$.

After we make a few remarks, the proof of case 2 goes exactly as the proof of Case 1B. In Case 2 there are two boxes $I_{n}^{i}, \widetilde{I}_{n}^{i} \subset I_{n+1}^{i}$, and the Center Theorem for $S_{n-1}$ implies that $s_{n-1} \geq l_{n-1}^{2}$.

Lemma 3.20. If $c_{n+1}^{r} \in S_{n+1}$ for $r=i, j$ then

(a) $\left|K^{*}+\left[c_{n+1}^{i}\right]\right| \leq 3 \delta_{n}^{1 / 2} / \delta_{n-1}$.

(b) $\max \left(\left|\left[c_{n+1}^{i}\right]-\left[c_{n+1}^{j}\right]\right|,\left|\left[c_{n+1}^{i}\right]+\left[c_{n+1}^{j}\right]+2 K^{*}\right|\right) \leq 6 \delta_{n}^{1 / 2} / \delta_{n-1}$.

Proof. We use (0.8) and the definition of $c_{n+1}^{i}$ to prove part (a). Part (b) follows immediately from part (a).

Since $c_{n}^{i} \in S_{n}$, we have $\left|E_{n}^{i}\left(K^{*}\right)-E^{*}\right| \leq \delta_{n} \sqrt{E^{*}+1}$. By (H2), (H9) we get $\left|E_{n}^{i}(K)-E^{*}\right| \leq \operatorname{cst}\left(\delta_{n}^{1 / 2} / \delta_{n-1}\right) \sqrt{E^{*}+1}$ for every $K$ in the interval $\left|K-K^{*}\right| \leq$ $6 \delta_{n}^{1 / 2} / \delta_{n-1}$. If we transform the equation $H\left(I_{n}^{i}\right) \psi_{n}^{i}=E_{n}^{i} \psi_{n}^{i}$ from $I_{n}^{i}$ to $\widetilde{I}_{n}^{i}$ we see that $\widetilde{E}_{n}^{i}(K)=E_{n}^{i}\left(-K-\left[c_{n}^{i}\right]-\left[\tilde{c}_{n}^{i}\right]\right)$ is an eigenvalue in $\sigma\left(H\left(\widetilde{I}_{n}^{i}\right)\right)$ which is near $E^{*}$. We use $\psi_{n}^{i}$ and $\tilde{\psi}_{n}^{i}$ as trial wave functions for $H\left(I_{n+1}^{i}\right)$ to get two eigenvalues $E_{n+1}^{i}(K)$ and $\mathscr{E}_{n+1}^{i}(K) \in \sigma\left(H\left(I_{n+1}^{i}\right)\right)$ which obey

$$
\left|E_{n+1}^{i}(K)-E^{*}\right| \leq \operatorname{cst}\left(\delta_{n}^{1 / 2} / \delta_{n-1}\right) \sqrt{E^{*}+1}
$$

and

$$
\left|\mathscr{E}_{n+1}^{i}(K)-E^{*}\right| \leq \operatorname{cst}\left(\delta_{n}^{1 / 2} / \delta_{n-1}\right) \sqrt{E^{*}+1} .
$$

We are now in the same setup as subcase $1 \mathrm{~B}$ and the rest of the proof will be omitted.

\section{Proof of the main Results}

In this section we will prove our main results.

Main Lemma. For $\varepsilon$ sufficiently small and for almost every $K$, every polynomially bounded eigenfunction of the operator $H(K)=\varepsilon \Delta+V(K)$ decays exponentially fast.

To establish the Main Lemma we will follow the proof given in [6]. First we will show that inside large enough boxes there must be points of $S_{n}$. Then the Center Theorem will give us annuli around these boxes which do not intersect $S_{n}$. Finally, the Decay Theorem will force the Green's function and the wave function to decay at points in the annulus. We will piece together annuli to prove that $\psi$ decays for all $|x|$ large. To accomplish this we need to set some notation and prove two lemmas. 
Definition 1. We call $E$ a generalized eigenvalue if $E$ satisfies the equation $H \psi=E \psi$ where $\psi$ is polynomially bounded.

Definition 2. We define $B(l)$ to be a square box of length $l$ centered at the origin of $\mathbf{Z}^{2}$. Let $\Lambda_{n} \equiv B\left(\frac{1}{2} l_{n+1}\right)$ and $A_{n} \equiv B\left(2 l_{n+2}\right) \backslash B\left(\frac{1}{2} l_{n+1}\right)$ where $l_{n}$ is the length scale defined in the introduction.

Lemma 4.1. Let $E^{*}$ be a generalized eigenvalue for $H\left(K^{*}\right)$; then there exists an integer $N\left(K^{*}, E^{*}\right)$ such that

$$
S_{n}\left(K^{*}, E^{*}\right) \cap \Lambda_{n} \neq \varnothing \text { for } n \geq N\left(K^{*}, E^{*}\right) .
$$

Proof. Assume not. Then there exists a sequence $n_{i} \rightarrow \infty$ such that

$$
S_{n_{i}}\left(K^{*}, E^{*}\right) \cap \Lambda_{n_{i}}=\varnothing .
$$

Fix any $x \in \mathbf{Z}^{2}$ and choose $i$ large enough so that $x \in \Lambda_{n_{i}}$ and $\operatorname{dist}\left(x, \partial \Lambda_{n_{i}}\right) \geq$ $l_{n_{i}}^{5 / 6}$. We will show that $\psi \equiv 0$ and therefore not an eigenfunction.

To see this we restrict the equation $H \psi=E^{*} \psi$ to the sets $\Lambda_{n_{i}}$ :

$$
\psi=\left(H\left(\Lambda_{n_{i}}\right)-E^{*}\right)^{-1}\left(\Gamma_{n_{i}} \psi\right) .
$$

This gives us

$$
\psi(x)=\sum_{y \in \partial \Lambda_{n_{i}}}\left(H\left(\Lambda_{n_{i}}-E^{*}\right)^{-1}(x, y)\left(\Gamma_{n_{i}} \psi\right)(y) .\right.
$$

Since $|x-y| \geq l_{n_{i}}^{5 / 6}$, the Decay Theorem and the polynomial boundedness of $\psi$ imply

$$
|\psi(x)| \leq \operatorname{cst} l_{n_{i}}^{p} \exp \left(-\left(\gamma_{0} / 2\right) l_{n_{i}}^{5 / 6}\right) \rightarrow 0 \quad \text { as } i \rightarrow \infty .
$$

Therefore $\psi(x) \equiv 0$ for all $x$.

Lemma 4.2. There exists a set $\mathscr{K}$ of Lebesgue measure zero with the following property:

If $E^{*}$ is a generalized eigenvalue for $H\left(K^{*}\right)$ where $K^{*} \notin \mathscr{K}$, then there exists an integer $N\left(K^{*}, E^{*}\right)$ such that $S_{n}\left(K^{*}, E^{*}\right) \cap A_{n}=\varnothing$ for $n \geq N\left(K^{*}, E^{*}\right)$. Proof. For any value of $E$, we define

$$
B_{n}(E) \equiv\left\{K \in \mathbf{R}: S_{n}(K, E) \cap A_{n} \neq \varnothing, S_{n}(K, E) \cap \Lambda_{n} \neq \varnothing\right\} .
$$

Let $C_{n} \equiv \bigcup_{E}\left(B_{n}(E)\right)$. If we show that $\sum_{n=0}^{\infty} \mu\left(C_{n}\right)$ converges then it is easy to see that

$$
\mu\left(\bigcap_{m=0}^{\infty} \bigcup_{n \geq m} C_{n}\right)=0 .
$$

Define $\mathscr{K}=\bigcap_{m=0}^{\infty} \bigcup_{n \geq m} C_{n}$. Let $E^{*}$ be any generalized eigenvalue for $H\left(K^{*}\right)$ and suppose that $K^{*} \notin \mathscr{K}$. Since $K^{*} \notin \mathscr{K}$ there must be an integer $N\left(K^{*}, E^{*}\right)$ such that $K^{*} \notin B_{n}\left(E^{*}\right)$ for $n \geq N\left(K^{*}, E^{*}\right)$. By Lemma 4.1 
we may assume that $S_{n}\left(K^{*}, E^{*}\right) \cap \Lambda_{n} \neq \varnothing$ for $n \geq N\left(K^{*}, E^{*}\right)$; therefore by definition of $B_{n}\left(E^{*}\right)$ we must have $S_{n}\left(K^{*}, E^{*}\right) \cap A_{n}=\varnothing$ for $n \geq N\left(K^{*}, E^{*}\right)$.

It remains to show that $\sum \mu\left(C_{n}\right)<\infty$. In particular, we will prove that $\mu\left(C_{n}\right) \leq l_{n+2}^{4} \delta_{n}^{1 / 3}$. To see this let $K \in C_{n}$. Then $K \in B_{n}(E)$ for some $E$; therefore we have points $a \in S_{n} \cap A_{n}, b \in S_{n} \cap \Lambda_{n}$ where $|a-b| \leq 2 l_{n+2}$. By the Center Theorem for $S_{n}$ and (0.2) it follows that

$$
|[a]+[b]+2 K| \leq 2 \delta_{n}^{1 / 2} / \delta_{n-1} .
$$

Therefore $K$ belongs to an interval of measure $O\left(\delta_{n}^{1 / 2} / \delta_{n-1}\right)$, but this interval depends on $E$ and $K$ since $a, b$ depend on $E$ and $K$. However, there are at most $O\left(l_{n+2}^{4}\right)$ points $a, b \in B\left(2 l_{n+2}\right)$, thus

$$
\mu\left(C_{n}\right) \leq \operatorname{cst} l_{n+2}^{4} \delta_{n}^{1 / 2} / \delta_{n-1} .
$$

We are now ready to prove the Main Lemma.

Proof (of Main Lemma). Choose $x \in B\left(l_{n+2}\right) \backslash B\left(l_{n+1}\right) \subset A_{n}$ where $n$ is large enough so that Lemma 4.2 applies. If we restrict the equation $H \psi=E^{*} \psi$ to $A_{n}$ we get

$$
\left.\psi=\left(H\left(A_{n}\right)-E^{*}\right)^{-1}\right)\left(\Gamma\left(A_{n}\right) \psi\right) .
$$

The Decay Theorem, Lemma 4.2, and the polynomial boundedness of $\psi$ give us

$$
|\psi(x)| \leq \operatorname{cst} e^{-\left(\gamma_{0} / 16\right)|x|} .
$$

With the help of the Main Lemma we can now go on to the proof of Theorems 1 and 2. For the proof of Theorem 1 see [1].

Theorem 2. For $\varepsilon$ sufficiently small, the operator

$$
-d^{2} / d x^{2}+\varepsilon(\cos x+\cos (\alpha x+\vartheta))
$$

has no point spectrum.

To prove Theorem 2 we will need the following lemma.

Lemma 4.3. Fix $E^{*}$. Then for almost every $K \in \mathbf{R}$, there exists an integer $N(K)$ such that $S_{n}\left(K, E^{*}\right) \cap \Lambda_{n}=\varnothing$ for $n \geq N(K)$.

Proof. If we define

$$
B_{n}=\left\{K \in \mathbf{R}: S_{n}\left(K, E^{*}\right) \cap \Lambda_{n} \neq \varnothing\right\},
$$

then the set $\mathscr{K}=\bigcap_{m=0}^{\infty} \bigcup_{n \geq m} B_{n}$ will be the set of measure zero we need to prove the lemma. To prove that this set has measure zero we define

$$
B_{n}^{i}=\left\{K \in \mathbf{R}: c_{n}^{i} \in S_{n}\left(K, E^{*}\right) \cap \Lambda_{n}\right\}
$$

which decomposes $B_{n}$; i.e., $B_{n}=\bigcup_{\Lambda_{n}} B_{n}^{i}$. If we can show that $\sum \mu\left(B_{n} \cap I\right)$ converges (for intervals $I$ ), then by the Borel-Cantelli theorem it follows that 
$\mu(\mathscr{K} \cap I)=0$ and therefore $\mu(\mathscr{K})=0$. To finish the proof we need to establish the estimate

$$
\mu\left(B_{n}^{i} \cap I\right) \leq \operatorname{cst}\left(\delta_{n}^{1 / 4}\right) /\left(\delta_{n-1}^{3 / 2}\right) .
$$

Let $K^{*} \in B_{n}^{i}$. Then $c_{n}^{i} \in S_{n}\left(K^{*}, E^{*}\right) \cap \Lambda_{n}$; therefore there exists an eigenvalue $E_{n}^{i}\left(K^{*}\right) \in \sigma\left(H\left(I_{n}^{i}\right)\right)$ such that

$$
\left|E_{n}^{i}\left(K^{*}\right)-E^{*}\right| \leq \delta_{n} \sqrt{E^{*}+1} .
$$

By induction we know that $E_{n}^{i}(K)$ is defined in the interval $\left|K-K^{*}\right| \leq \operatorname{cst} \delta_{n-1}^{3 / 2}$, and by induction hypotheses $(\mathrm{H} 3)$ and $(\mathrm{H} 10)$ we can show that $\mid E_{n}^{i}\left(K \pm \delta_{n}^{1 / 4}\right)-$ $E^{*} \mid \geq \delta_{n} \sqrt{E^{*}+1}$. From this it follows that every interval of length $O\left(\delta_{n-1}^{3 / 2}\right)$ can intersect $B_{n}^{i}$ in a set of measure at most $O\left(\delta_{n}^{1 / 4}\right)$.

Now we can prove Theorem 2 .

Proof (of Theorem 2). Suppose that we have a solution to the equation

$$
-d^{2} \psi / d x^{2}+\varepsilon(\cos x+\cos (\alpha x+\vartheta)) \psi=E^{*} \psi .
$$

Let $\phi_{m n}^{K}=e^{i m \vartheta} \hat{\psi}(K+m+n \alpha)$ where $\hat{\psi}$ is the Fourier transform of $\psi$. Then $\phi^{K}$ satisfies the eigenvalue equation

$$
H(K) \phi^{K}=(\varepsilon \Delta+V(K)) \phi^{K}=E^{*} \phi^{K}
$$

on the lattice $\mathbf{Z}^{2}$. Later we will prove that $\phi^{K}$ is polynomially bounded for almost all $K$. If we use this fact with Lemma 4.3 and an argument similar to the one used in the proof of lemma 4.1 , we can show that $\phi^{K} \equiv 0$ for almost every $K$. Thus $\hat{\psi}(K)=0$ for almost every $K$, which implies that $\psi=0$ and not an eigenfunction.

Now we need only show that $\phi^{K}$ is polynomially bounded for almost every $K$. To see this we note that since $\psi \in L_{2}$ we have

$$
\int\left|\phi_{m n}^{K}\right|^{2} d K=\int|\psi(K)|^{2} d K=1
$$

therefore

$$
\int \sum_{m, n} \frac{\left|\phi_{m n}^{K}\right|^{2}}{1+|m|^{4}+|n|^{4}} d K=\sum_{m, n} \frac{1}{1+|m|^{4}+|n|^{4}}<\infty .
$$

This implies that $\sum_{m, n}\left(\left|\phi_{m n}^{K}\right|^{2} /\left(1+|m|^{4}+|n|^{4}\right)\right)$ is bounded by some constant $C_{K}$ for almost every $K$; therefore $\phi^{K}$ is polynomially bounded for almost every $K$.

\section{APPENDIX A}

Lemma. Let $E(K)$ be defined and twice differentiable for every $K$ in the interval $\left|K-K^{*}\right| \leq \eta \leq \frac{1}{2}$. Suppose that there is a point $K_{s}$ in the interval such that 
$E\left(K_{s}-\delta K\right)=E\left(K_{s}+\delta K\right)$. We also assume that $\left|E^{\prime}\right| \leq s$ implies $\left|E^{\prime \prime}\right| \geq t$ and $E^{\prime \prime}$ has a unique sign. Then

(a)

$$
\left|E\left(K_{2}\right)-E\left(K_{1}\right)\right| \geq \min (s / 2, t / 4) \min \left\{\begin{array}{l}
\left|K_{2}-K_{1}\right|^{2}, \\
\left|K_{2}+K_{1}-2 K_{s}\right|^{2}
\end{array}\right.
$$

for any points $K_{1}, K_{2}$ belonging to the interval $\left|K-K^{*}\right| \leq \eta$.

(b)

$$
\left|E^{\prime}(K)\right| \geq \min \left\{\begin{array}{l}
s, \\
t\left|K-K_{s}\right| .
\end{array}\right.
$$

Proof. Without loss of generality, we may consider the case $\left|E^{\prime}\right| \leq s$ implies $E^{\prime \prime} \geq t$. By symmetry we must have $E^{\prime}\left(K_{s}\right)=0$; therefore $E^{\prime \prime}\left(K_{s}\right) \geq t$. Let $K_{d}$ be the largest point with the following property:

$$
E^{\prime \prime}(K) \geq t \text { for } K_{s} \leq K \leq K_{d} .
$$

This implies that $E(K)$ is an increasing function to the right of the symmetry point. By definition of $K_{d}$ we have $E^{\prime \prime}\left(K_{d}+\delta K\right)<t$ for $\delta K$ small; therefore $E^{\prime}\left(K_{d}+\delta K\right) \geq s$. This inequality must hold for every $K>K_{d}$ or else we would have a point $K$ where $E^{\prime \prime}(K) \geq t>0$. This is impossible. (See Figure A.) Therefore

$$
E^{\prime}(K) \geq s \text { for } K>K_{d} .
$$

We now split up the proof into cases.

Case 1. $K_{s} \leq K_{1} \leq K_{2} \leq K_{d}$.

$$
\begin{aligned}
\left|E\left(K_{2}\right)-E\left(K_{1}\right)\right| & =E\left(K_{2}\right)-E\left(K_{1}\right) \\
& =E^{\prime}\left(K_{1}\right)\left(K_{2}-K_{1}\right)+\frac{1}{2} E^{\prime \prime}(\widehat{K})\left(K_{2}-K_{1}\right)^{2} \\
& \geq(t / 2)\left|K_{2}-K_{1}\right|^{2} .
\end{aligned}
$$

Case 2. $K_{d} \leq K_{1} \leq K_{2}$.

$$
\begin{aligned}
\left|E\left(K_{2}\right)-E\left(K_{1}\right)\right| & =E\left(K_{2}\right)-E\left(K_{1}\right)=E^{\prime}(\widehat{K})\left(K_{2}-K_{1}\right) \\
& \geq s\left|K_{2}-K_{1}\right| \geq s\left|K_{2}-K_{1}\right|^{2} .
\end{aligned}
$$
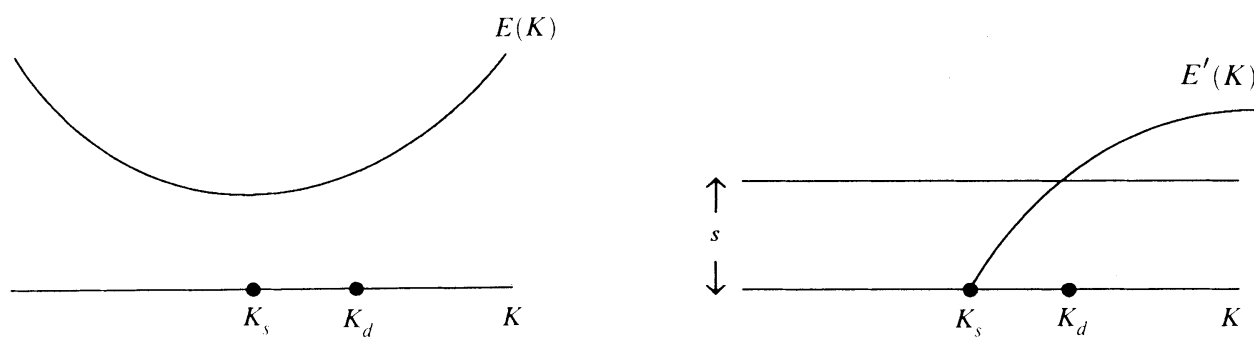

FIgURE A 
Case 3. $K_{s} \leq K_{1} \leq K_{d} \leq K_{2}$.

$$
\left|E\left(K_{2}\right)-E\left(K_{1}\right)\right|=E\left(K_{2}\right)-E\left(K_{d}\right)+E\left(K_{d}\right)-E\left(K_{1}\right) .
$$

By Cases 1 and 2 we get

$$
\begin{aligned}
\left|E\left(K_{2}\right)-E\left(K_{1}\right)\right| & \geq s\left|K_{2}-K_{d}\right|^{2}+(t / 2)\left|K_{d}-K_{1}\right|^{2} \\
& \geq \frac{1}{2} \min (s, t / 2)\left|K_{2}-K_{1}\right|^{2} \\
& =\min (s / 2, t / 4)\left|K_{2}-K_{1}\right|^{2} .
\end{aligned}
$$

Case 4. $K_{1} \leq K_{s} \leq K_{2}$. We use the symmetry of $E(K)$ to write

$$
\left|E\left(K_{2}\right)-E\left(K_{1}\right)\right|=\left|E\left(K_{2}\right)-E\left(2 K_{s}-K_{1}\right)\right|,
$$

which by Cases $1-3$ gives us

$$
\left|E\left(K_{2}\right)-E\left(K_{1}\right)\right| \geq \min (s / 2, t / 4)\left|K_{2}+K_{1}-2 K_{s}\right|^{2} .
$$

To prove part (b), we need to consider two cases.

Case 1. $K_{s} \leq K \leq K_{d}$.

$$
E^{\prime}(K)=E^{\prime}\left(K_{s}\right)+E^{\prime \prime}(\widehat{K})\left(K-K_{s}\right) \geq t\left|K-K_{s}\right| .
$$

Case 2. $K_{d} \leq K$. In this case we have $\left|E^{\prime}(K)\right| \geq s$.

\section{APPENDIX B}

Lemma. Suppose $H \psi=E \psi$ and $H \Psi=\mathscr{E} \Psi$, where $\mathscr{E}$ is the closest eigenvalue to $E$. We assume that the eigenfunctions are normalized and everything is defined for $K$ in the interval $\left|K-K^{*}\right| \leq \eta$. Then

(a) The eigenvalues and eigenfunctions can be chosen to be analytic functions of $K$.

(b) $E^{\prime}(K)=\left\langle\psi, V^{\prime} \psi\right\rangle$.

(c)

$$
\begin{aligned}
E^{\prime \prime}(K) & =2+2\left\langle V^{\prime} \psi,(E-H)_{\perp}^{-1} V^{\prime} \psi\right\rangle \\
& =2+\frac{2\left\langle\psi, V^{\prime} \Psi\right\rangle^{2}}{E-\mathscr{E}}+2\left\langle V^{\prime} \psi,(E-H)_{\perp \perp}^{-1} V^{\prime} \psi\right\rangle
\end{aligned}
$$

at all points where $E(K) \neq \mathscr{E}(K)$.

Remark. From the Spectral Theorem we have

$$
G_{\perp} \equiv(E-H)_{\perp}^{-1}=\sum_{E_{\alpha} \neq E}\left(E-E_{\alpha}\right)^{-1} P\left(E_{\alpha}\right)
$$

where $P\left(E_{\alpha}\right)$ are projections. Similarly, we write

$$
G_{\perp \perp} \equiv(E-H)_{\perp \perp}^{-1}=\sum_{E_{\alpha} \neq E, \mathscr{E}}\left(E-E_{\alpha}\right)^{-1} P\left(E_{\alpha}\right) .
$$

Proof. If the eigenvalues never cross, part (a) obviously holds. When there are level crossings, we can always find a way to label our eigenvalues so that they 
are analytic. This follows from the fact that our operator $H(K)$ is analytic and selfadjoint. See [10].

To prove part (b) we differentiate the equation $H \psi=E \psi$ to get $H^{\prime} \psi+$ $H \psi^{\prime}=E^{\prime} \psi+E \psi^{\prime}$. Note that $H^{\prime}=V^{\prime}$, and then take the inner product with $\psi$ yielding

$$
\left\langle\psi, V^{\prime} \psi\right\rangle+\left\langle\psi, H \psi^{\prime}\right\rangle=E^{\prime}\langle\psi, \psi\rangle+E\left\langle\psi, \psi^{\prime}\right\rangle .
$$

Since the second term on the left $\left\langle\psi, H \psi^{\prime}\right\rangle=\left\langle H \psi, \psi^{\prime}\right\rangle=E\left\langle\psi, \psi^{\prime}\right\rangle$ is canceled by the same term on the right, we get $E^{\prime}=\left\langle\psi, V^{\prime} \psi\right\rangle$.

To establish part (c) we differentiate the result in part (b) to get

$$
E^{\prime \prime}=\left\langle\psi^{\prime}, V^{\prime} \psi\right\rangle+\left\langle\psi, V^{\prime \prime} \psi\right\rangle+\left\langle\psi, V^{\prime} \psi^{\prime}\right\rangle=\left\langle\psi, V^{\prime \prime} \psi\right\rangle+2\left\langle\psi, V^{\prime} \psi^{\prime}\right\rangle .
$$

By definition of $V$ we have $V^{\prime \prime}=2 I$ (where $I$ is the identity matrix); therefore

$$
E^{\prime \prime}=2+2\left\langle\psi, V^{\prime} \psi^{\prime}\right\rangle \text {. }
$$

To complete the proof we must calculate $\psi^{\prime}$. We rewrite $(\mathrm{B} 1)$ as $(E-H) \psi^{\prime}=$ $V^{\prime} \psi-E^{\prime} \psi$, thus

$$
\psi^{\prime}=(E-H)_{\perp}^{-1}\left(V^{\prime} \psi-E^{\prime} \psi\right)=(E-H)_{\perp}^{-1} V^{\prime} \psi .
$$

If we put (B2) back into our last equation for $E^{\prime \prime}$ we get

$$
E^{\prime \prime}(K)=2+2\left\langle V^{\prime} \psi,(E-H)_{\perp}^{-1} V^{\prime} \psi\right\rangle
$$

We can further express

$$
(E-H)_{\perp}^{-1}=(E-H)_{\perp \perp}^{-1}+P(\mathscr{E}) /(E-\mathscr{E}),
$$

and if we note that $P(\mathscr{E}) V^{\prime} \psi=\left\langle\psi, V^{\prime} \Psi\right\rangle \Psi$, we substitute (B4) into (B3) to get

$$
E^{\prime \prime}=2+2\left\langle\psi, V^{\prime}(E-H)_{\perp \perp}^{-1} V^{\prime} \psi\right\rangle+2\left\langle\psi, V^{\prime} \Psi\right\rangle^{2} /(E-\mathscr{E})
$$

\section{APPENDIX C}

Decay Theorem. If $\Lambda$ is $n+1$ regular and $S_{n+1}\left(K^{*}, E^{*}\right) \cap \Lambda=\varnothing$ then

$$
\left|(H(\Lambda)-E)^{-1}(x, y)\right| \leq \frac{\mathrm{cst}}{\delta_{0} \sqrt{E^{*}+1}} e^{-\gamma_{n+1}|x-y|}
$$

provided $|x-y| \geq l_{n+1}^{5 / 6},\left|K-K^{*}\right| \leq 4 \delta_{n+1}^{3 / 2}$, and $\left|E-E^{*}\right| \leq\left(\delta_{n+1} / 5\right) \sqrt{E^{*}+1}$, where $\gamma_{0} \geq \gamma_{n+1} \geq \gamma_{0} / 2$.

Proof. Let $\Lambda$ be $n+1$ regular and suppose $\bar{S}_{n} \cap \Lambda$ consists of just one point $c_{n+1}^{i}$. Then there exists a box $\widehat{I}_{n+1}^{i} \subset I_{n+1}^{i}$ with the following properties:

(a) $S_{n} \cap\left(\Lambda \backslash \widehat{I}_{n+1}^{i}\right)=\varnothing$.

(b) $\Lambda \backslash \widehat{I}_{n+1}^{i}$ is $n$ regular.

(c) $\operatorname{dist}\left(\partial \widehat{I}_{n+1}^{i},\{x, y\}\right) \geq l_{n}^{5 / 6}$.

(d) length of $\widehat{I}_{n+1}^{i}=O\left(l_{n+1}^{1 / 2}\right)$. 
By (0.7) we have

$$
\begin{aligned}
G(x, y) & \equiv(H(\Lambda)-E)^{-1}(x, y)=\left[G_{\Gamma}+G_{\Gamma} \Gamma G\right](x, y) \\
& =\left[G_{\Gamma}+G_{\Gamma} \Gamma G_{\Gamma}+G_{\Gamma} \Gamma G \Gamma G_{\Gamma}\right](x, y)
\end{aligned}
$$

where $\Gamma=\partial \widehat{I}_{n+1}^{i}$. Notice that the second term on the right in (C2) vanishes unless $\Gamma$ separates $x$ and $y$. By property $(\mathrm{d})$, we see that it is impossible for $x$ and $y$ simultaneously to belong to $\widehat{I}_{n+1}^{i}$. If we assume that $x, y \in \Lambda \backslash \widehat{I}_{n+1}^{i}$, then $G_{\Gamma}=G\left(\Lambda \backslash \widehat{I}_{n+1}^{i}\right)$ decays by induction. To estimate $G$ we assume

$$
\left|G\left(u^{\prime}, v^{\prime}\right)\right| \leq 4\left(\delta_{n+1} \sqrt{E^{*}+1}\right)^{-1} \text { for } u^{\prime}, v^{\prime} \in \widehat{I}_{n+1}^{i} .
$$

Therefore by $(\mathrm{C} 2)$ we get

$$
\begin{aligned}
|G(x, y)| \leq & \frac{\mathrm{cst}}{\delta_{0} \sqrt{E^{*}+1}} e^{-\gamma_{n}|x-y|} \\
& +\frac{\mathrm{cst}}{\delta_{0} \sqrt{E^{*}+1}} e^{-\gamma_{n}|x-y|} e^{\operatorname{cst} \gamma_{n}\left(l_{n+1}^{1 / 2}+l_{n+1}^{2 / 3}\right)} \\
\leq & \frac{\mathrm{cst}}{\delta_{0} \sqrt{E^{*}+1}} e^{-\gamma_{n+1}|x-y|}
\end{aligned}
$$

where

$$
\gamma_{n+1}=\gamma_{n}\left(1-\operatorname{cst} \frac{l_{n+1}^{1 / 2}+l_{n+1}^{2 / 3}}{l_{n+1}^{5 / 6}}\right) .
$$

If $x \in \widehat{I}_{n+1}^{i}$ and $y \notin \widehat{I}_{n+1}^{i}$, we use the resolvent identity $G=G_{\Gamma}+G \Gamma G_{\Gamma}$ to obtain a similar result.

It remains to prove $(\mathrm{C} 3)$. By alternate application of the two resolvent identities

$$
G=G_{\bar{\Gamma}}+G_{\bar{\Gamma}} \bar{\Gamma}_{G} \quad \text { and } \quad G=G_{\Gamma}+G_{\Gamma} \Gamma G
$$

(where $\bar{\Gamma}=\partial I_{n+1}^{i}$ ) we get

$$
G\left(u^{\prime}, v^{\prime}\right)=\left[G_{\bar{\Gamma}}+G_{\bar{\Gamma}} \bar{\Gamma} G_{\Gamma}+G_{\bar{\Gamma}} \bar{\Gamma} G_{\Gamma} \Gamma G_{\bar{\Gamma}}+\cdots\right]\left(u^{\prime}, v^{\prime}\right) .
$$

Since $G_{\bar{\Gamma}}=G\left(I_{n+1}^{i}\right)$ and by assumption $\operatorname{dist}\left(\sigma\left(H\left(I_{n+1}^{i}, K^{*}\right)\right), E^{*}\right) \geq$ $\delta_{n+1} \sqrt{E^{*}+1}$, we get $\left|G_{\bar{\Gamma}}\left(u^{\prime}, v^{\prime}\right)\right| \leq 2\left(\delta_{n+1} \sqrt{E^{*}+1}\right)^{-1}$ for $K$ near $K^{*}$ and $E$ near $E^{*}$. Note that in all nonvanishing terms of $(\mathrm{C} 4)$ we have $G_{\Gamma}=G\left(\Lambda \backslash \widehat{I}_{n+1}^{i}\right)$, and since $\operatorname{dist}(\Gamma, \bar{\Gamma}) \geq l_{n}$ we have $\left|\bar{\Gamma} G\left(\Lambda \backslash \widehat{I}_{n+1}^{i}\right) \Gamma\right| \leq e^{-\gamma_{n} l_{n}}$. Therefore

$$
\left|G\left(u^{\prime}, v^{\prime}\right)\right| \leq 4\left(\delta_{n+1} \sqrt{E^{*}+1}\right)^{-1} \text {. }
$$

The general case where $\Lambda$ contains many points $c_{n+1}^{i} \in \bar{S}_{n}$ is treated using the block resolvent expansion as explained in [15].

\section{APPENDIX D}

Lemma. Let $B(l)$ be a square box of length $l$. Then there exists an $n$ regular box $\Lambda$ such that

$$
B\left(l_{n+1}\right) \subset \Lambda \subset B\left(2 l_{n+1}\right)
$$

whose perimeter is bounded by $l_{n+1}^{2}$. 
Proof. It suffices to show that we can pass a broken line across a rectangle of dimension $\frac{1}{2} l_{n+1} \times 2 l_{n+1}$ which misses every box $I_{m}^{i}$ for $m \leq n$.

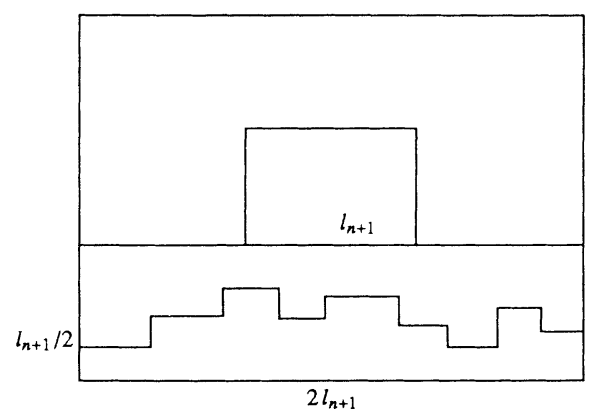

The proof will be by induction on $n$. Assume we are given a rectangle with dimensions $\frac{1}{2} l_{n+1} \times 2 l_{n+1}$. By the Center Theorem for $S_{n}$, it is possible to put a strip of width $l_{n} / 2$ across our rectangle so that it misses every box $I_{n}^{i}$. We now break up the strip into rectangles which by induction have paths that avoid $I_{m}^{i}$ for $m \leq n-1$. The length of the path is bounded by induction.

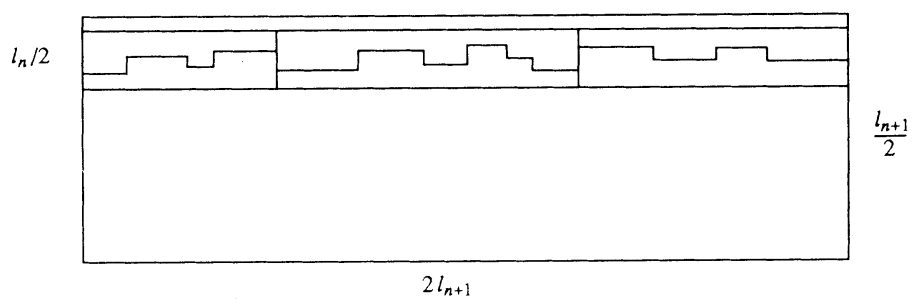

Remark. It is possible to choose the box $\Lambda$ to be symmetric about its center.

Acknowledgment. I would like to thank Professor T. Spencer for introducing me to the subject of this paper and for the many discussions which led to the final result.

\section{REFERENCES}

1. J. M. Berezanskii, Expansion in eigenfunctions of self adjoint operators, Transl. Math. Monographs, vol. 17, Amer. Math. Soc., Providence, R.I., 1968.

2. F. Bloch, Über die Quantenmechanik der Elektronen in Kristallgittern, Z. Phys. 52 (1928), 555 .

3. F. Delyon, Y. Levy, and B. Souillard, Comm. Math. Phys. 100 (1985), 463.

4. E. I. Dinaburg and Ya. G. Sinai, The one-dimensional Schrödinger equation with a quasiperiodic potential, Functional Anal. Appl. 9 (1975), 279.

5. J. Fröhlich and T. Spencer, Absence of diffusion in the Anderson tight binding model for large disorder or low energy, Comm. Math. Phys. 88 (1983), 151.

6. J. Fröhlich, F. Martinelli, E. Scoppola, and T. Spencer, Localization in the Anderson tight binding model, Comm. Math. Phys. 101 (1985), 21. 
7. G. Gallavotti, The elements of mechanics, Springer-Verlag, New York, 1983.

8. I. Gold'sheid, S. Molchanov, and L. Pastur, Pure point spectrum of stochastic one-dimensional Schrödinger operators, Functional Anal. Appl. 11 (1977), 1.

9. R. Johnson and J. Moser, The rotation number for almost periodic potentials, Comm. Math. Phys. 84 (1982), 403.

10. T. Kato, Perturbation theory for linear operators, 2nd ed., Springer, New York, 1976.

11. J. Moser and J. Pöschel, An extension of a result by Dinaburg and Sinai on quasi-periodic potentials, Comment. Math. Helv. 59 (1984), 39.

12. B. Simon, Almost periodic Schrödinger operators: a review, Adv. in Appl. Math. 3 (1982), 463.

13. B. Simon and T. Wolff, Comm. Pure Appl. Math. 39 (1986), 75.

14. Ya. G. Sinai, Anderson localization for the one dimensional difference Schrödinger operator with quasiperiodic potential, J. Statist. Phys. 46 (1987), 861.

15. T. Spencer, The Schrödinger equation with a random potential: a mathematical review, Lecture Notes, Les Houches Summer School, 1984.

16. T. Spencer, J. Fröhlich, and P. Wittwer, Localization for a class of one dimensional quasiperiodic Schrödinger operators, preprint, 1987.

17. E. C. Titchmarsh, Eigenfunction expansions associated with second-order differential equations, Oxford Univ. Press, 1962.

18. G. André and S. Aubry, Analyticity breaking and Anderson localization in incommensurate lattices, Ann. Israel Phys. Soc. 3 (1980), 133.

19. S. Aubry, The new concept of transition by breaking of analyticity, Solid State Sci. 8 (1978), 264.

20. F. Delyon, Absence of localization in the almost Mathieu equation, J. Phys. A 20 (1987), L21.

21. P. Lax, Lecture notes on Hilbert space, New York Univ., 1970.

Department of Mathematics and Computer Science, Drew University, Madison, New JERSEY 07940 